\title{
Effective Field Theory of Dark Matter from membrane inflationary paradigm
}

\author{
Sayantan Choudhury ${ }^{a}$ Arnab Dasgupta $^{b}$ \\ ${ }^{a}$ Department of Theoretical Physics, Tata Institute of Fundamental Research, Homi Bhabha \\ Road, Colaba, Mumbai - 400005, India ${ }^{1}$ \\ ${ }^{b}$ Institute of Physics, Sachivalaya Marg, Bhubaneswar, Odisha - 751005, India \\ E-mail: sayantan@theory.tifr.res.in, arnab.d@iopb.res.in
}

ABSTRACT: In this article, we have studied the cosmological and particle physics constraints on dark matter relic abundance from effective field theory of inflation from tensor-to-scalar ratio $(r)$, in case of Randall-Sundrum single membrane (RSII) paradigm. Using semi-analytical approach we establish a direct connection between the dark matter relic abundance $\left(\Omega_{D M} h^{2}\right)$ and primordial gravity waves $(r)$, which establishes a precise connection between inflation and generation of dark matter within the framework of effective field theory in RSII membrane. Further assuming the UV completeness of the effective field theory perfectly holds good in the prescribed framework, we have explicitly shown that the membrane tension, $\sigma \leq \mathcal{O}\left(10^{-9}\right) M_{p}^{4}$, bulk mass scale $M_{5} \leq \mathcal{O}(0.04-0.05) M_{p}$, and cosmological constant $\tilde{\Lambda}_{5} \geq-\mathcal{O}\left(10^{-15}\right) M_{p}^{5}$, in RSII membrane plays the most significant role to establish the connection between dark matter and inflation, using which we have studied the features of various mediator mass scale suppressed effective field theory "relevant operators" induced from the localized $s, t$ and $u$ channel interactions in RSII membrane. Taking a completely model independent approach, we have studied an exhaustive list of tree-level Feynman diagrams for dark matter annihilation within the prescribed setup and to check the consistency of the obtained results, further we apply the constraints as obtained from recently observed Planck 2015 data and Planck+BICEP2+Keck Array joint datasets. Using all of these derived results we have shown that to satisfy the bound on, $\Omega_{D M} h^{2}=0.1199 \pm 0.0027$, as from Planck 2015 data, it is possible to put further stringent constraint on $r$ within, $0.01 \leq r \leq 0.12$, for thermally averaged annihilation cross-section of dark matter, $\langle\sigma v\rangle \approx \mathcal{O}\left(10^{-28}-10^{-27}\right) \mathrm{cm}^{3} / \mathrm{s}$, which are very useful to constrain various membrane inflationary models.

KEYwORDS: Inflation, Membrane paradigm, Braneworld gravity, Effective Field Theory, Dark Matter.

\footnotetext{
${ }^{1}$ Presently working as a Visiting (Post-Doctoral) fellow at DTP, TIFR, Mumbai, Alternative E-mail: sayanphysicsisi@gmail.com.
} 


\section{Contents}

1 Introduction 1

2 An overview of membrane paradigm $\quad 6$

3 Inflationary constraints on dark Matter abundance from membrane paradigm

4 Effective Field Theory of dark matter from membrane paradigm 17

4.1 Dirac dark matter: spin-0 mediator 17

$\begin{array}{lll}4.1 .1 & \text { s-channel analysis } & 17\end{array}$

4.1.2 t/u- channel analysis 21

4.2 Dirac Dark matter: spin-1 mediator 24

4.2.1 s-channel analysis 24

$\begin{array}{lll}4.2 .2 \mathrm{t} / \mathbf{u} \text { - channel analysis } & 26\end{array}$

4.3 Majorana dark Matter: spin-1 mediator 30

$\begin{array}{lll}4.3 .1 & \text { s-channel analysis } & 30\end{array}$

4.3.2 t/ $\mathbf{u}$ - channel analysis 33

4.4 Complex scalar dark matter: spin-0 mediator 35

4.5 Complex scalar dark matter: spin-1 mediator 38

4.6 Complex vector dark matter: spin-0 mediator 41

4.7 Complex vector dark matter: spin-1 mediator 44

4.8 Complex scalar dark matter: spin - 1/2 mediator 47

4.9 Real Scalar dark matter: spin-1/2 mediator 50

4.10 Complex Vector dark matter: spin-1/2 mediator 52

$\begin{array}{lll}5 & \text { Summary } & 55\end{array}$

\section{Introduction}

Identifying the nature of dark matter, which will have profound consequences in the context of cosmology and particle physics. At present a significant research is being devoted into the search for dark matter in:

1. Indirect searches where the prime objective is to detect the products of dark matter annihilations or decays around the milky way [1], 
2. Direct searches where the prime target is to detect the scattering between dark matter and heavy mesons [2],

3. Collider searches (specifically at LHC) where the main goal is to search for mono-jet [3-6] and mono-photon [7-10].

Despite the unknown nature of dark matter, from theoretical point of view Weakly Interacting Massive Particle (WIMP) is the most studied favoured candidate, whose estimated thermal relic abundance is consistent with the present observed data. Particle physics beyond the Standard Model is the mostly renowned area in this context which provides such a dark matter candidate in a model dependent way. To study the signatures of the dark matter candidate in a model independent way one of the powerful approaches is Effective Field Theory framework $[11-13]^{1}$ in theoretical physics, using which the various $s, t$ and $u$ channel interactions of dark matter candidate and the known Standard Model field contents are parametrized by a set of mediator mass scale suppressed effective non-renormalizable Wilsonian operators, usually generated from integrating out the heavy mediator from the theory [20-33]. Also the effective field theory framework is a very sophisticated theoretical way to describe physical phenomena occurring at a specified energy scale in terms of all possible allowed interactions. The effective field theory framework suggests that the effective operators in principle can explain the direct, indirect detection and collider search of WIMPs, which require an interaction of WIMPs with Standard Model sector in the effective theory. Most importantly, the effective field theory framework is very advantageous as it can be testable through various experiments ${ }^{2}$.

Apart from the huge success of the effective field theory prescription, it is important to mention here that the justifiability of the framework depends on the separation between the characteristic energy scale of the dark matter annihilation process and scale of underlying microscopic interactions. In the context of indirect dark matter searches, the annihilation of the non-relativistic dark matter particle contents in the galaxy occur with momentum transfer comparable to the dark matter mass. On the other hand, in case of direct searches the momentum transfer process involved in the scattering of dark matter with heavy nuclei are comparable to $\mathrm{keV}$ scale [11-13]. In both of the cases, it is possible to implement the effective field theory technique, provided the UV cut-off of the prescribed theory is larger than the typical momentum transfer. However, in the context of collider searches, the associated energy scale involved is very high and the Wilsonian effective operators exist at the scale beyond the validity of effective field theory framework itself.

\footnotetext{
${ }^{1}$ See ref. [14] for the review on effective field theory techniques. See also ref. [15] for the effective field theory construction on Randall-Sundrum (RSII) 3 membrane. To know more about effective field theory of inflation see ref. [16-19].

${ }^{2}$ In the present day research, the effective field theory framework is one of the important branches at LHC experiment for the dark matter searches in the mono-jet and mono-photon channels [11-13].
} 
Thus before applying the effective field theory prescription it is important to know about full UV complete theory, the range of applicability and consistency of framework in the specific context ${ }^{3}$. In fig. (1), we have explicitly shown the schematic representation of effective field theory setup in membrane paradigm which shows the complete algorithm of the described methodology in this paper.

In the present context the effective field theory in four dimension is originated from varieties of string theories, specifically the 10-dimensional $E_{8} \otimes E_{8}$ heterotic string theory is one of the strongest candidates, which contain the standard model of particle physics and is related to an 11-dimensional superstring theory written on the orbifold $R^{10} \otimes S^{1} / Z_{2}$. Within this UV complete field theoretic setup, the standard model particle species are confined to the 4-dimensional space-time which is the sub-manifold of $R^{4} \otimes S^{1} / Z_{2}$. On the other hand, the graviton degrees of freedom propagate in the bulk space-time. To visualize in a more simplified way one can think about a situation where we consider a 5-dimensional problem in which the matter fields are confined to the 4-dimensional space-time while gravity acts in 5 dimensional bulk space-time. Randall-Sundrum (RSII) single membrane is one of the frameworks in which our observable universe in embedded on 3-membrane, where effective field

${ }^{3}$ In the matter sector incorporating the effects of quantum correction through the interaction between heavy and light field sector and finally integrating out the heavy degrees of freedom from the $4 \mathrm{D}$ Effective Field Theory picture the matter action [34, 35], which admits a systematic expansion within the light sector can be written as:

$$
\begin{aligned}
& S_{\text {matter }}[\chi, \Psi]=\int d^{4} x \sqrt{-{ }^{(4)} g}\left[\mathcal{L}_{D M}[\chi]+\mathcal{L}_{\text {heavy }}[\Psi]+\mathcal{L}_{\text {int }}[\chi, \Psi]\right] \\
& \stackrel{\text { Remove } \Psi}{\longrightarrow} e^{i S_{D M}[\chi]}=\int[\mathcal{D} \Psi] e^{i S_{\text {matter }}[\chi, \Psi]} \\
& S_{D M}[\chi]=\int d^{4} x \sqrt{-{ }^{(4)} g}\left[\mathcal{L}_{D M}[\chi]+\sum_{\alpha} J_{\alpha}(g) \frac{\mathcal{O}_{\alpha}[\chi]}{M_{p}^{\Delta_{\alpha}-4}}\right]
\end{aligned}
$$

where $J_{\alpha}(g)$ are dimensionless Wilson coefficients that depend on the couplings g of the UV theory, and $\mathcal{O}_{\alpha}[\chi]$ are local operators of dimension $\Delta_{\alpha}$. This procedure typically generates all possible effective operators $\mathcal{O}_{\alpha}[\chi]$ consistent with the symmetries of the UV theory. Also $\mathcal{L}_{D M}[\chi]$ and $\mathcal{L}_{\text {heavy }}[\Psi]$ describe the part of total Lagrangian density $\mathcal{L}$ involving only the light and heavy fields, and $\mathcal{L}_{\text {int }}[\chi, \Psi]$ includes all possible interactions involving both sets of fields within Effective Field Theory prescription. After removal of heavy degrees of freedom the effective action is decomposed into a renormalizable part and a sum of non-renormalizable corrections appearing through the Wilsonian operators $\mathcal{O}_{\alpha}[\chi]$. Such operators of dimensions less than four are called "relevant operators" $[35,36]$. They dominate in the IR and become small in the UV. In 4D Effective Field Theory the operators of dimensions greater than four are called irrelevant operators. These operators become small in the IR regime, but dominate in the UV end. However such corrections are extremely hard to compute and at the same time the theoretical origin of all such corrections is not at all clear till now as it completely belongs to the hidden sector of the theory. One of the possibilities of the origin of such hidden sector heavy field is higher dimensional Superstring Theory or its low energy supergravity version. Such a higher dimension setups dimensionally reduced to the 4D Effective Field Theory version via various compactifications. 


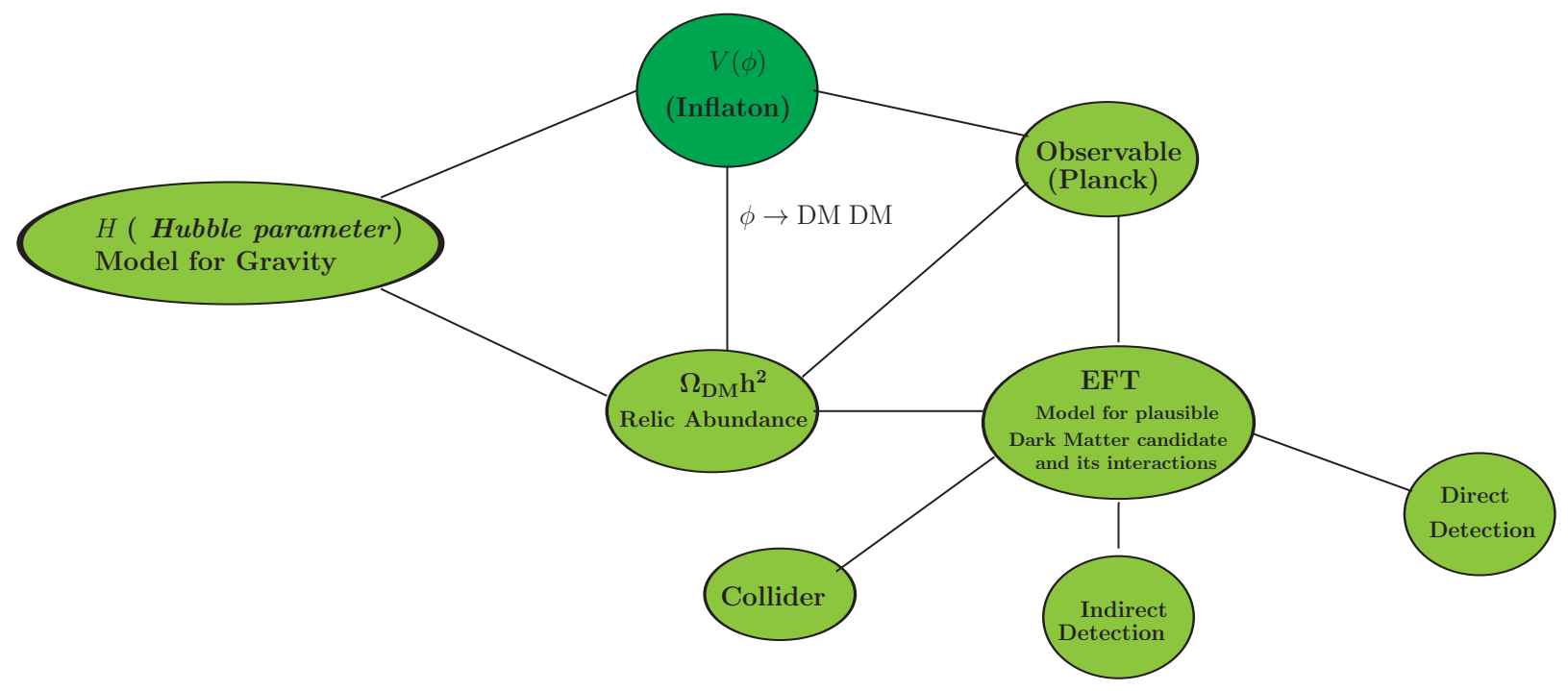

Figure 1. Schematic representation of effective field theory setup in membrane paradigm which shows the complete algorithm of the described methodology in this paper.

theory prescription is valid [34]. In field theory language one can interpret this membrane to be the boundary of 5-dimensional anti-de Sitter $\left(A d S_{5}\right)$ bulk spacetime and the corresponding effective field theory at the boundary is equivalent to the four dimensional Conformal Field Theory $\left(C F T_{4}\right)$. In this paper, we consider RSII membrane paradigm as a theoretical probe using which we have studied the validity and consequences from effective field theory framework to explain inflation and dark matter in the light of Planck 2015 data.

The prime objective of this paper is to establish a theoretical constraint to explicitly show a direct connection between the dark matter relic abundance $\left(\Omega_{D M} h^{2}\right)$ and primordial gravity waves $(r)$, which establish a precise connection between inflation and generation of dark matter within the framework of effective field theory in RSII membrane paradigm.

Throughout the analysis of the paper we assume:

1. Inflaton field $\phi$ and all the effective field theory interactions are localized in the membrane of RSII set up and also minimally coupled to the gravity sector at the single membrane.

2. Here in the RSII membrane paradigm the extra dimension " $y$ " is non-compact for which the covariant formalism is applicable. Also in our discussion we use the bulk and membrane parameters: 1) $M_{5}$ represents the $5 \mathrm{D}$ quantum gravity cut-off scale, 2) $\Lambda_{5}$ represents the $5 \mathrm{D}$ bulk cosmological constant, 3) 3 membrane has a positive membrane tension $\sigma$ and it is localized at the position of orbifold point $y=0[34,37-41]$. The exact connecting relationship between the bulk and membrane parameters, $M_{5}, \Lambda_{5}$ and $\sigma$ are explicitly mentioned 
in the later section of this article. Also for the sake of simplicity, in the RSII membrane set-up, during cosmological analysis, one can choose the following sets of parameters to be free:

- Bulk cosmological constant $\Lambda_{5}$ is the most important parameter of RSII set up [34, 37-41]. Only the upper bound of $\Lambda_{5}$ is fixed to validate the Effective Field Theory framework within the prescribed set up. Once I choose the value of $\Lambda_{5}$ below its upper bound value, the other two parameters$5 \mathrm{D}$ quantum gravity cut-off scale $M_{5}$ and the membrane tension $\sigma$ is fixed from their connecting relationship as discussed later. In this paper, we fix the values of all of these RSII membrane parameters by using Planck 2015 data and Planck+BICEP2/Keck Array joint constraints.

- The rest of the free parameters are explicitly appearing through the various couplings and mass of dark matter content in the effective theory.

3. Slow-roll prescription perfectly holds good, which is useful to estimate the tensor-to-scalar ratio $r$ from the RSII membrane inflationary paradigm.

4. Initial condition is guided via the Bunch-Davies vacuum.

5. The effective sound speed during inflation is fixed at $c_{S}=1$.

The plan of the paper is as follows.

- In section 2, we will briefly review the basic setup of RSII single membrane paradigm. Here we begin our discussion with the 5D action which contains the effective action localized at orbifold fixed point of the membrane. Finally, we will derive the Friedmann equation in the membrane, which is very very useful for further computation in the paper.

- In section 3, we will establish a theoretical constraint to explicitly show a direct connection between the dark matter relic abundance $\left(\Omega_{D M} h^{2}\right)$ and primordial gravity waves $(r)$, which establish a precise connection between inflation and generation of dark matter within the framework of effective field theory in RSII membrane paradigm.

- In section 4, we will explicitly study the details of Effective Field Theory of dark matter from membrane paradigm paradigm.

- In this paper we use various constraints arising from Planck 2015 data on the upper bound on tenor to scalar ratio, and the bound on dark matter abundance within $1.5 \sigma-2 \sigma$ statistical CL.

- I also mention that the GR limiting result $(\rho<<\sigma)$ and the difference between the high energy limit result $(\rho>>\sigma)$ of RSII. 
- Hence in section 4.1.1 and 4.1.2, we have studied the $s$ and $t / u$ channel interaction of the effective theory of Dirac dark matter with spin-0 mediator. Further, in section 4.2.1 and 4.2.2, we have studied the $s$ and $t / u$ channel interaction of the effective theory of Dirac dark matter with spin-1 mediator. Then, in section 4.3.1 and 4.3.2, we have studied the $s$ and $t / u$ channel interaction of the effective theory of Majorana dark matter with spin-1 mediator. After that, in section 4.4, we have studied the consequences from s-channel interaction of complex and real scalar dark matter with spin-0 mediator. Next, in section 4.5, we have studied the consequences from s-channel interaction of complex scalar and real vector dark matter with spin-1 mediator. Further, in section 4.6 and 4.7, we have studied the consequences from s-channel interaction of complex and real vector dark matter with spin-0 and spin-1 mediator respectively. Finally, in section 4.8, 4.9 and 4.10, we have studied the consequences from $\mathrm{t} / \mathrm{u}$-channel interaction of complex and real scalar and complex and real vector dark matter with spin-1/2 mediator.

- At the end, in section 5, we summarize our obtained results point-wise.

\section{An overview of membrane paradigm}

Let us start our discussion with a very brief introduction to membrane paradigm based on Randall-Sundrum single membrane (RSII) setup. The RSII single membrane setup and its most generalized version from a Minkowski membrane to a Friedmann- Robertson-Walker (FRW) membrane were derived as solutions in specific choice of coordinates of the 5D Einstein equations in the bulk, along with the junction conditions, which are applied at the $\mathbf{Z}_{2}$-symmetric single membrane. A broader perspective, with non-compact dimensions, can be obtained via the well known covariant Shiromizu-Maeda-Sasaki approach, in which the membrane and bulk metrics take its generalized structure [42]. The key point is to use the Gauss-Codazzi equations to project the $5 \mathrm{D}$ bulk curvature along the membrane using the covariant formalism. Here we start with the well known 5D Rundall-Sundrum (RSII) single membrane model action given by [43]:

$$
S_{R S}=\int d^{5} x \sqrt{-{ }^{(5)} g}\left[\frac{M_{5}^{3}}{2}{ }^{(5)} R-2 \Lambda_{5}+\mathcal{L}_{\text {bulk }}+\left(\mathcal{L}_{\text {membrane }}-\sigma\right) \delta(y)\right],
$$

where the extra dimension " $\mathrm{y}$ " is non-compact for which the covariant formalism is applicable. Here $M_{5}$ be the $5 \mathrm{D}$ quantum gravity cut-off scale, $\Lambda_{5}$ be the $5 \mathrm{D}$ bulk cosmological constant, $\mathcal{L}_{\text {bulk }}$ be the bulk field Lagrangian density, $\mathcal{L}_{\text {membrane }}$ signifies the Lagrangian density for the membrane field contents. It is important to mention the the scalar inflaton degree of freedom is embedded on the 3 membrane which has 
a positive membrane tension $\sigma$ and it is localized at the position of orbifold point $y=0$ in case of single membrane. The $5 \mathrm{D}$ field equations in the bulk, including explicitly the contribution of the RS single membrane is given by [44]:

$$
{ }^{(5)} G_{A B}=\frac{1}{M_{5}^{3}}\left[-\Lambda_{5}{ }^{(5)} g_{A B}+{ }^{(5)} T_{A B}+T_{\mu \nu}^{\text {membrane }} \delta_{A}^{\mu} \delta_{B}^{\nu} \delta(y)\right]
$$

where ${ }^{(5)} T_{A B}$ characterizes any $5 \mathrm{D}$ energy-momentum tensor of the gravitational sector within bulk spece-time. On the other hand the total energy-momentum tensor on the membrane is given by:

$$
T_{\mu \nu}^{\text {membrane }}=T_{\mu \nu}-\sigma g_{\mu \nu}
$$

where $T_{\mu \nu}$ is the energy-momentum tensor of particles and fields confined to the single membrane so that the constraint condition,

$$
T_{A B} n^{B}=0
$$

is valid in the present context. Let us consider further $y$ be a Gaussian normal coordinate, which is orthogonal to the single membrane, chosen to be placed at the orbifold point $y=0$ without loss of generality, so that an infinitesimal change along the direction of extra dimensional coordinate is represented by,

$$
n_{A} d X^{A}=d y
$$

where $n^{A}$ be the unit normal. In case of RS single membrane setup the 5D metric in terms of the induced metric on the family of $y=$ const. hyper-surfaces is locally represented by [44]:

$$
\begin{aligned}
{ }^{(5)} g_{A B} & =g_{A B}+n_{A} n_{B}, \\
{ }^{(5)} d s^{2} & =g_{\mu \nu}\left(x^{\alpha}, y\right) d x^{\mu} d x^{\nu}+d y^{2} .
\end{aligned}
$$

Here it is assumed that the metric $g_{\mu \nu}\left(x^{\alpha}, y\right)$ is non-factorizable in the most generalized prescription. In such a situation one can Taylor expand of the metric about the single membrane. In Gaussian normal coordinates after applying Taylor expansion one can write [44]:

$$
\begin{aligned}
g_{\mu \nu}\left(x^{\alpha}, y\right)= & g_{\mu \nu}\left(x^{\alpha}, 0\right)-\frac{1}{M_{5}^{3}}\left[T_{\mu \nu}+\frac{1}{3}(\sigma-T) g_{\mu \nu}\right]_{y=0+}|y| \\
& +\left[-\mathcal{E}_{\mu \nu}+\frac{1}{4 M_{5}^{6}}\left(T_{\mu \alpha} T_{\nu}^{\alpha}+\frac{2}{3}(\sigma-T) T_{\mu \nu}\right)\right. \\
& \left.+\frac{1}{6}\left(\frac{1}{6 M_{5}^{6}}(\sigma-T)^{2}-\Lambda_{5}\right) g_{\mu \nu}\right]_{y=0+} y^{2}+\ldots
\end{aligned}
$$


Now further integrating Equation (2.2) along the extra dimension within the interval $y=-\epsilon$ to $y=+\epsilon$, and after taking the limit $\epsilon \rightarrow 0$, leads to the well known IsraelDarmois junction conditions at the membrane:

$$
\begin{aligned}
g_{\mu \nu}^{+} & =g_{\mu \nu}^{-}, \\
K_{\mu \nu}^{+} & =-K_{\mu \nu}^{-}=-\frac{1}{2 M_{5}^{3}}\left[T_{\mu \nu}+\frac{1}{3}(\sigma-T) g_{\mu \nu}\right],
\end{aligned}
$$

where we define, $T=T^{\mu}{ }_{\mu}$ and and one can evaluate quantities on the membrane by taking the physical limit $y \rightarrow+0$. This further implies that the effective action on the 3 membrane can be expressed as:

$$
S_{\text {eff }}=\int d^{4} x \sqrt{-{ }^{(4)} g}\left[\frac{M_{p}^{2}}{2}\left({ }^{(4)} R-2 \Lambda_{4}\right)+\mathcal{L}_{\text {membrane }}+\Delta_{\mathcal{S}}-\Delta_{\mathcal{E}}\right]
$$

where $\Delta_{\mathcal{S}}$ and $\Delta_{\mathcal{E}}$ are the contributions from quadratic part of the energy-momentum tensor $T_{\mu \nu}$ and Weyl tensor respectively. Finally one can arrive at the 4-dimensional Einstein induced field equations on the single membrane given by [44, 45]:

$$
G_{\mu \nu}=-\Lambda_{4} g_{\mu \nu}+\frac{1}{M_{p}^{2}} T_{\mu \nu}+\left(\frac{8 \pi}{M_{5}^{3}}\right)^{2} \mathcal{S}_{\mu \nu}-\mathcal{E}_{\mu \nu}
$$

where $T_{\mu \nu}$ represents the energy-momentum on the single membrane, $\mathcal{S}_{\mu \nu}$ is a rank-2 tensor that contains contributions that are quadratic in the energy momentum tensor $T_{\mu \nu}$ represented by:

$$
\begin{aligned}
\mathcal{S}_{\mu \nu} & =\frac{M_{5}^{6}}{16 \pi^{2} M_{p}^{2} \sqrt{-{ }^{(4)} g}} \frac{\delta\left(\sqrt{-{ }^{(4)} g} \Delta_{\mathcal{S}}\right)}{\delta g^{\mu \nu}} \\
& =\frac{1}{12} T T_{\mu \nu}-\frac{1}{4} T_{\mu \alpha} T^{\alpha}{ }_{\nu}+\frac{1}{24} g_{\mu \nu}\left[3 T_{\alpha \beta} T^{\alpha \beta}-T^{2}\right] .
\end{aligned}
$$

and $\mathcal{E}_{\mu \nu}$ characterizes the projection of the 5-dimensional Weyl tensor on the 3 -membrane which is physically equivalent to the non-local contributions pressure and energy flux for a perfect fluid, given by:

$$
\begin{aligned}
\mathcal{E}_{\mu \nu} & =\frac{2}{\sqrt{-{ }^{(4)} g}} \frac{\delta\left(\sqrt{-{ }^{(4)} g} \Delta_{\mathcal{E}}\right)}{\delta g^{\mu \nu}} \\
& ={ }^{(5)} C_{A C B D} n^{C} n^{D} g_{\mu}{ }^{A} g_{\nu}{ }^{B}
\end{aligned}
$$

which is orthogonal to the unit normal vector $n^{B}$ represented via the following condition:

$$
\mathcal{E}_{A B} n^{B}=0=\mathcal{E}_{[A B]}=\mathcal{E}_{A}{ }^{A},
$$

which is the outcome of the Weyl tensor symmetries. 
In a cosmological framework, where the 3-membrane resembles our universe and the metric projected onto the membrane is an homogeneous and isotropic flat Friedmann-Robertson-Walker (FRW) metric, the Friedmann equation becomes [44, 45]:

$$
H^{2}=\frac{\Lambda_{4}}{3}+\frac{\rho}{3 M_{p}^{2}}+\left(\frac{4 \pi}{3 M_{5}^{3}}\right)^{2} \rho^{2}+\frac{\epsilon}{a^{4}},
$$

where $\epsilon$ is an integration constant. The four and five-dimensional cosmological constants are related by:

$$
\Lambda_{4}=\frac{4 \pi}{M_{5}^{3}}\left(\Lambda_{5}+\frac{4 \pi}{3 M_{5}^{3}} \sigma^{2}\right),
$$

where $\sigma$ is the 3-membrane tension. Within RS setup the quantum gravity cut-off scale i.e. the 5D Planck mass and effective 4D Planck mass are connected through the visible membrane tension as:

$$
M_{5}^{3}=\sqrt{\frac{4 \pi \sigma}{3}} M_{p}
$$

Assuming that, as required by observations, the $4 \mathrm{D}$ cosmological constant is negligible $\Lambda_{4} \approx 0$ in the early universe the localized visible membrane tension is given by:

$$
\sigma=\sqrt{-\frac{3}{4 \pi} M_{5}^{3} \Lambda_{5}}=\sqrt{-24 M_{5}^{3} \tilde{\Lambda}_{5}}>0
$$

where $\tilde{\Lambda}_{5}$ be the scaled 5D bulk cosmological constant defined as:

$$
\tilde{\Lambda}_{5}=\frac{\Lambda_{5}}{32 \pi}<0
$$

Also the last term in Eq. (2.16) rapidly becomes redundant after inflation sets in, the Friedmann equation in RSII membrane becomes [44, 45]:

$$
H^{2}=\frac{\rho}{3 M_{p}^{2}}\left(1+\frac{\rho}{2 \sigma}\right)
$$

where $\sigma$ be the positive membrane tension, $\rho$ signifies the energy density of the inflaton field $\phi$ and $M_{p}=2.43 \times 10^{18} \mathrm{GeV}$ be the reduced 4D Planck mass. Using Eq (2.19) in Eq (2.18), the 5D quantum gravity cut-off scale can be expressed in terms of 5D cosmological constant as:

$$
M_{5}^{3}=\sqrt[3]{-\frac{4 \pi \Lambda_{5}}{3}} M_{p}^{4 / 3}=\sqrt[3]{-\frac{128 \pi^{2} \tilde{\Lambda}_{5}}{3}} M_{p}^{4 / 3} .
$$

In the low energy limit $\rho<<\sigma$ in which standard GR framework can be retrieved. On the other hand, in the high energy regime $\rho>>\sigma$ as the effect of membrane correction factor is dominant which is my present focus in this paper. 


\section{Inflationary constraints on dark Matter abundance from membrane paradigm}

In this section we will discuss the inflationary constraints on dark matter from membrane paradigm in detail. To serve this purpose let us first start with the total energy density $\rho$, which is localized in the single membrane and can be expressed as:

$$
\rho=\rho_{r}+\rho_{\phi},
$$

where $\rho_{r}$ and $\rho_{\phi}$ represent the energy density during radiation and inflation, and can be written as:

$$
\begin{aligned}
\rho_{r} & =\frac{\pi^{2}}{30} g_{*} \frac{m_{\chi}^{4}}{\Theta^{4}} \\
\rho_{\phi} & =g_{\phi} m_{\chi}^{4} x\left(\frac{x}{2 \pi \Theta}\right)^{3 / 2} e^{-x \Theta}
\end{aligned}
$$

where the parameters $x$ and $\Theta$ are defined as:

$$
\begin{aligned}
x & =\frac{m_{\phi}}{m_{\chi}}, \\
\Theta & =\frac{m_{\chi}}{T},
\end{aligned}
$$

and $m_{\phi}$ is the mass of inflaton field, $T$ is the temperature and $m_{\chi}$ is the mass of the Dark Matter candidate. It is important to mention here that, the dynamics of the radiation and the inflaton field are governed by the following version of continuity equations as:

$$
\begin{gathered}
\frac{d \rho_{r}}{d t}+4 H \rho_{r}=\Gamma_{\phi} \rho_{\phi} \\
\frac{d \rho_{\phi}}{d t}+3 H \rho_{\phi}=-\Gamma_{\phi} \rho_{\phi}
\end{gathered}
$$

Now further combining (3.6) with (3.7), we get the following simplified expression:

$$
\mathcal{F}(\Theta)=\frac{d \Theta}{d t}=H\left[\frac{2 \Theta\left(8 e^{x \Theta} g_{*} \pi^{7 / 2}+45 \sqrt{2}(x / 2)^{5 / 2} \Theta^{5}\right)}{16 e^{x \Theta} g_{*} \pi^{7 / 2}+15 \sqrt{2}(x / 2)^{5 / 2} \Theta^{5}(2 x+3)}\right] .
$$

where $\mathrm{H}$ is the Hubble parameter, which can be expressed via Friedmann equation as:

$$
H^{2}=\frac{\left(\rho_{r}+\rho_{\phi}\right)}{3 M_{p}^{2}}\left[1+\frac{\left(\rho_{r}+\rho_{\phi}\right)}{2 \sigma}\right],
$$

where $M_{p}$ be the reduced/ effective Planck mass, $M_{p}=2.43 \times 10^{18} \mathrm{GeV}$. It is important to note that, in the present context, the scale of membrane inflation $V_{\text {inf }}$ can be expressed in terms of tensor-to-scalar ratio $r$ as:

$$
V_{i n f} \approx \tilde{\Delta} \times\left(\frac{r}{0.12}\right)
$$


where $\tilde{\Delta}$ is around GUT scale, which is constrained by Planck 2015 and Planck 2015+ BICEP2/Keck Array joint data set. For numerical estimations we fix,

$$
\tilde{\Delta} \approx 1.96 \times 10^{16} \mathrm{GeV} .
$$

Now for the sake of simplicity within membrane paradigm we define the following dimensionless parameter:

$$
\alpha=\frac{V_{i n f}}{\sigma} \approx \frac{\tilde{\Delta}}{\sigma} \times\left(\frac{r}{0.12}\right) .
$$

Further substituting $\mathrm{Eq}$ (3.12) in Eq (3.9) we get the following expression for the Hubble parameter in terms of the dimensionless parameter $\alpha$ as:

$$
H^{2}=\frac{\left(\rho_{r}+\rho_{\phi}\right)}{3 M_{p}^{2}}\left[1+\frac{0.12 \alpha}{2 r \tilde{\Delta}^{4}}\left(\rho_{r}+\rho_{\phi}\right)\right] .
$$

Henceforth, we will use Eq (3.13) for the computation of dark matter abundance.

Additionally, for completeness it is important to note that, to validate the effective field theory prescription within the framework of small field models of inflation, the field excursion is sub-Planckian i.e.

$$
|\Delta \phi|=\left|\phi_{e}-\phi_{c m b}\right|<M_{p}
$$

where $\phi_{e}$ and $\phi_{c m b}$ represent the inflaton field values at the end of inflation and at horizon crossing respectively. For detailed derivation of this important result see ref.[34] ${ }^{4}$, where using model independent semi-analytical approach we have derived the field excursion formula for RSII single membrane in terms of inflationary observables and the final result is perfectly consistent with the effective field theory prescription. Using this result the model independent bound on the membrane tension, the 5D cut-off scale and 5D bulk cosmological constant can be computed as ${ }^{5}$ :

$$
\begin{aligned}
\sigma & \leq \mathcal{O}\left(10^{-9}\right) M_{p}^{4}, \\
M_{5} & \leq \mathcal{O}(0.04-0.05) M_{p}, \\
\tilde{\Lambda}_{5} & \geq-\mathcal{O}\left(10^{-15}\right) M_{p}^{5},
\end{aligned}
$$

which we have followed during the numerical estimation performed in this paper.

\footnotetext{
${ }^{4}$ The similar bound we have derived in the context of GR in ref. [46-50]. Also see ref. [51-53], where the numerical proof is given in the context of MSSM inflation to justify the validity of the bound on field excursion.

5 Additionally it is important to note that, in order to recover the observational successes of general relativity (GR), the high-energy regime where significant deviations occur must take place before nucleosynthesis. Table-top tests of Newtons laws put the lower bound on the membrane tension and 5D Planck scale as: $\sigma>\mathcal{O}\left(2.86 \times 10^{-86}\right) M_{p}^{4}$ and $M_{5}>\mathcal{O}\left(4.11 \times 10^{-11}\right) M_{p}$. But such lower bound will not be able to produce large tensor-to-scalar ratio as required by the upper bound of Planck 2015 data.
} 
Now in the context of RSII membrane paradigm, the Bolztmann equation for the dark matter relic abundance can be expressed as :

$$
\frac{d Y_{\mathrm{DM}}}{d \Theta}=\frac{s(\Theta)\langle\sigma v\rangle}{\mathcal{F}(\Theta)}\left[1+\frac{1}{3} \frac{d \ln g_{s}}{d \ln x}\right]\left(\left(Y_{\mathrm{DM}}^{E Q}\right)^{2}-Y_{\mathrm{DM}}^{2}\right)
$$

where all the informations of RSII membrane is encoded in the generalized function $\mathcal{F}(\Theta)$, which is defined earlier in Eq (3.8). The Boltzmann equation and the relic abundance calculation for the standard GR case is given in appendix B. In Eq (3.18), the dark matter relic abundance $Y_{\mathrm{DM}}$ is defined as:

$$
Y_{\mathrm{DM}}=\frac{n_{\mathrm{DM}}}{s(\Theta)},
$$

where $n_{\mathrm{DM}}$ is the number density of dark matter content and and $s(\Theta)$ characterizes the entropy density of the universe defined as:

$$
s(\Theta)=\frac{S}{a^{3}}=\frac{2 \pi^{2}}{45} g_{*} m_{\chi}^{3} \Theta^{-3},
$$

where $S$ and $a$ represent the total entropy of the universe and scale factor respectively. Also $g_{\star}$ represents the total number of degrees of freedom.

Additionally, it is important to note that, in $\mathrm{Eq}$ (3.18), $\langle\sigma v\rangle$ represents the thermally averaged total annihilation cross-section times the Moller velocity, where we have summed over final and averaged over initial spins, $Y_{\mathrm{DM}}^{E Q}$ signifies the dark matter relic abundance at matter and radiation equality and also $g_{s}$ represents the effective number of relativistic degrees of freedom defined as:

$$
\sqrt{g_{*}}=\sqrt{g_{s}}\left(1+\frac{1}{3} \frac{d \ln g_{s}}{d \ln \Theta}\right)
$$

which is in principle function of the parameter $x=m_{\phi} / m_{\chi}$.

Further assuming the fact that the inflaton field is very heavy compared to the dark matter content $\left(m_{\phi}>>m_{\chi}\right)$, Eq (3.18) can be expressed as:

$$
\frac{d Y_{\mathrm{DM}}}{d \Theta}=\frac{s\langle\widetilde{\sigma} v\rangle(\Theta)}{\Theta H_{G R}(\Theta)}\left[1+\frac{1}{3} \frac{d \ln g_{s}}{d \ln \Theta}\right]\left[\left(Y_{\mathrm{DM}}^{E Q}\right)^{2}-Y_{\mathrm{DM}}^{2}\right]
$$

where we introduce three functions $\langle\widetilde{\sigma} v\rangle(\Theta), H_{G R}(\Theta)$ and characteristic function in RSII membrane, $f_{\text {membrane }}(\Theta)$, which are defined as:

$$
\begin{aligned}
\langle\widetilde{\sigma} v\rangle(\Theta) & =\frac{\langle\sigma v\rangle}{f_{\text {membrane }}(\Theta)}, \\
\Sigma(\Theta) & =\sqrt{\frac{g_{*}}{90}} \frac{\pi m_{\chi}^{2}}{M_{p} \Theta^{2}}, \\
f_{\text {membrane }}(\Theta) & =\left[1+\frac{0.12 \alpha}{2 r \tilde{\Delta}^{4}}\left\{\rho_{r}(\Theta)+\rho_{\phi}(\Theta)\right\}\right]^{1 / 2}, \\
& =\left[1+\frac{0.12 \alpha}{2 r \tilde{\Delta}^{4}}\left(\frac{\pi^{2}}{30} g_{*} \frac{m_{\mathrm{DM}}^{4}}{\Theta^{4}}+g_{\phi} m_{\chi}^{4} x\left(\frac{x}{2 \pi \Theta}\right)^{3 / 2} e^{-x \Theta}\right)\right]^{1 / 2}
\end{aligned}
$$




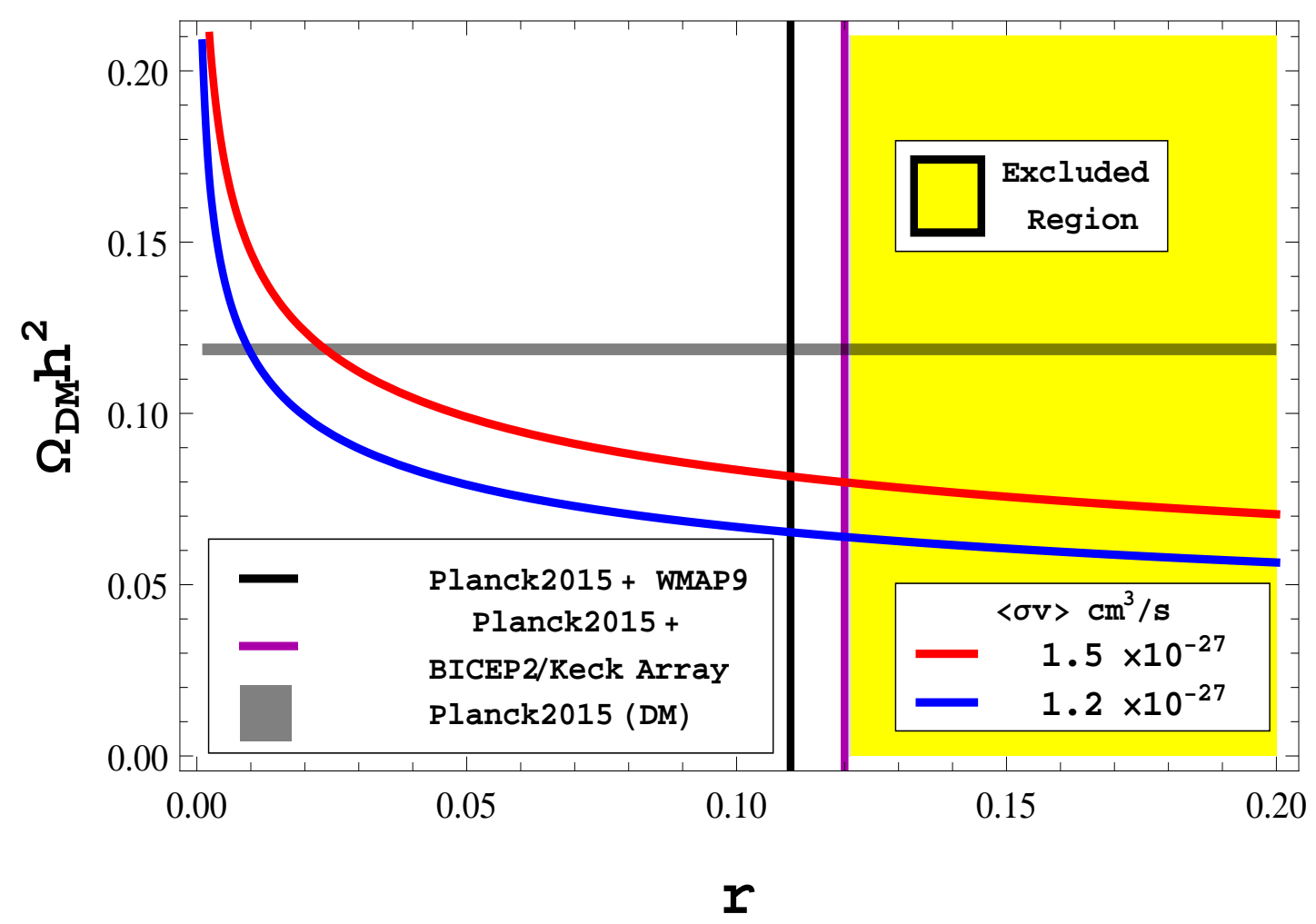

Figure 2. The above plot shows the dependence of relic abundance $\Omega_{\mathrm{DM}} h^{2}$ with tensor to scalar ratio $r$ taking $\alpha=1.77 \times 10^{64}$ for different thermally averaged cross-section.

In the present context, the thermally averaged cross-section is given by the following generalized expression [54]:

$$
\langle\sigma v\rangle=\frac{1}{8 m^{4} T K_{2}^{2}(m / T)} \int_{4 m^{2}}^{\infty} \sigma\left(s-4 m^{2}\right) \sqrt{s} K_{1}(\sqrt{s} / T) d s
$$

where $K_{i}$ 's are the modified Bessel function of the $i^{\text {th }}$ order. In the non-relativistic regime Eq (3.26) can be recast as:

$$
\langle\sigma v\rangle=\frac{2 \Theta^{3 / 2}}{\pi^{1 / 2}} \int_{0}^{\infty} \sigma v \epsilon^{1 / 2} e^{-\Theta \epsilon} d \epsilon=\frac{\Theta^{1 / 2}}{\sqrt{4 \pi}} \int_{0}^{\infty}(\sigma v) v^{2} e^{\left(-x v^{2} / 4\right)} d v
$$

where we use the following expressions:

$$
\begin{aligned}
& \epsilon=p_{\mathrm{rel}}^{2} /\left(4 m^{2}\right)=\frac{(v / 2)^{2}}{1-(v / 2)^{2}}, \\
& s=\frac{4 m^{2}}{1-v^{2} / 4} .
\end{aligned}
$$

Now, the dark matter relic abundance can be found out by

$$
\Omega_{\mathrm{DM}} h^{2}=\left(\frac{m_{\chi} s Y_{\mathrm{DM}}}{3 H^{2} M_{p}^{2}}\right)_{\text {today }} h^{2} \simeq \frac{1.07 \times 10^{9} \mathrm{GeV}^{-1}}{J\left(\Theta_{F}\right) g_{*}^{1 / 2} M_{p}}
$$




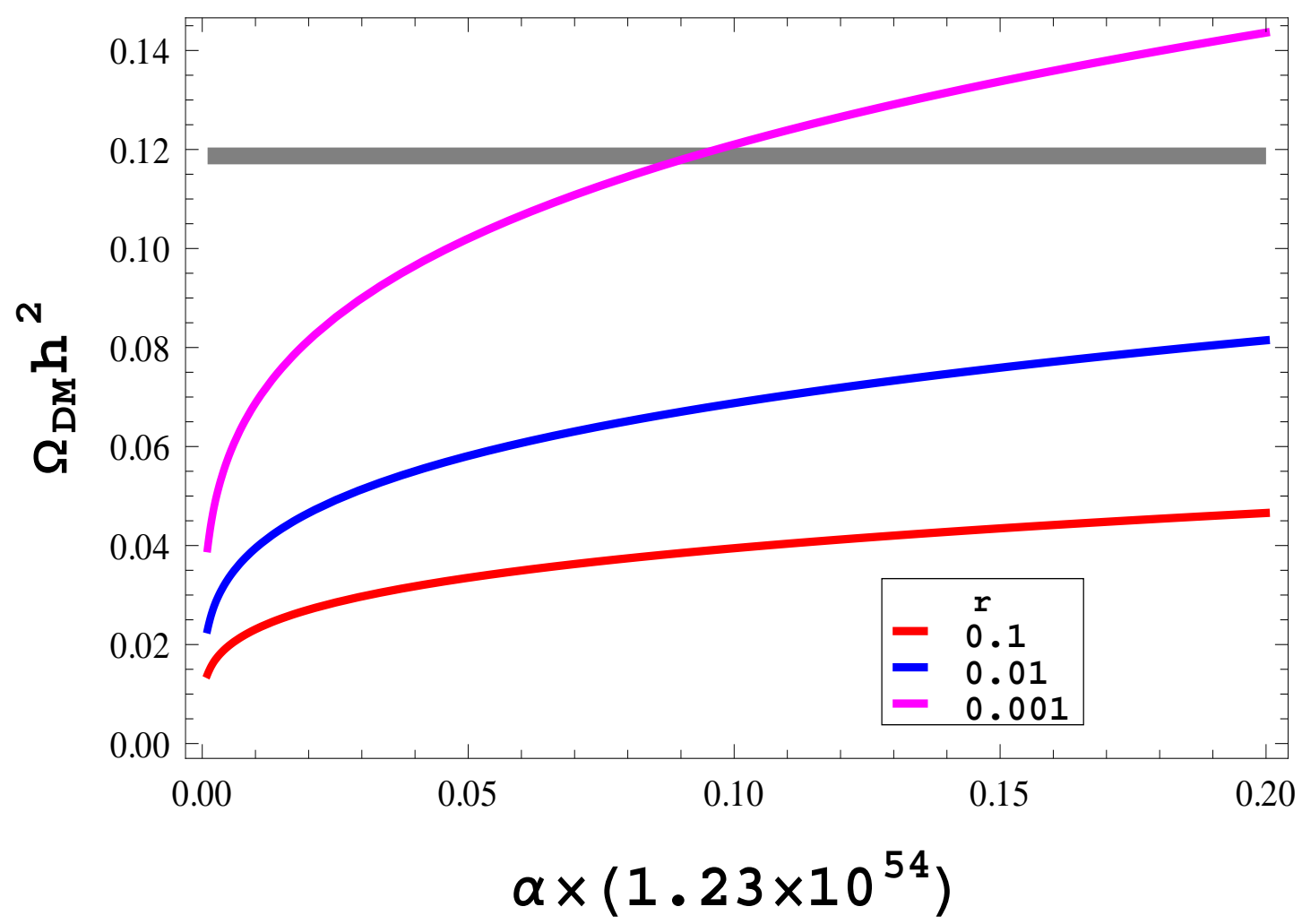

Figure 3. The above plot shows the dependence of relic abundance $\Omega_{\mathrm{DM}} h^{2}$ with tensor to scalar ratio $r$ taking $\alpha=1.77 \times 10^{64}$ for different thermally averaged cross-section.

where using s-wave approximation the newly introduced factor $J\left(\Theta_{F}\right)$ is defined as:

$$
\begin{aligned}
J\left(\Theta_{F}\right) & =\int_{\Theta_{F}}^{\infty} d \Theta \frac{\langle\widetilde{\sigma} v\rangle(\Theta)}{\Theta^{2}} \\
& \approx \int_{\Theta_{F}}^{\infty} d \Theta \int_{0}^{\infty} d v \frac{v^{2}\left(a+b v^{2}\right)}{\sqrt{4 \pi \Theta}} \frac{e^{-\Theta v^{2} / 4}}{f_{\text {membrane }}(\Theta)} \\
& =\int_{\Theta_{F}}^{\infty}\left(a+\frac{3}{2} \frac{b}{\Theta}\right) \frac{d \Theta}{\Theta^{2} f_{\text {membrane }}(\Theta)}
\end{aligned}
$$

where $\Theta_{F}$ is the freeze-out temperature which can be calculated by numerically solving the following transcendental equation:

$$
\Theta_{F}=\ln \left(\frac{0.038 g_{\mathrm{DM}} m_{\chi} M_{p}\langle\widetilde{\sigma} v\rangle(\Theta)}{g_{*}^{1 / 2} \Theta_{F}^{1 / 2}}\right)=\ln \left(\frac{0.038 g_{\mathrm{DM}} m_{\chi} M_{p}\langle\sigma v\rangle}{g_{*}^{1 / 2} f_{\text {membrane }}\left(\Theta_{F}\right) \Theta_{F}^{1 / 2}}\right) .
$$

In the context of RSII membrane paradigm when the energy density of the dark matter content is very very large compared to the membrane tension i.e. $\rho_{D M}=$ $\rho_{\chi}>>\sigma$, then one can write the following simplified expressions for the memebrane 
characteristic function $f_{\text {membrane }}(\Theta)$ as:

$$
\begin{aligned}
f_{\text {membrane }}(\Theta) & \approx\left[\frac{0.12 \alpha}{2 r \tilde{\Delta}^{4}}\left\{\rho_{r}(\Theta)+\rho_{\phi}(\Theta)\right\}\right]^{1 / 2}, \\
& =\left[\frac{0.12 \alpha}{2 r \tilde{\Delta}^{4}}\left(\frac{\pi^{2}}{30} g_{*} \frac{m_{\mathrm{DM}}^{4}}{\Theta^{4}}+g_{\phi} m_{\chi}^{4} x\left(\frac{x}{2 \pi \Theta}\right)^{3 / 2} e^{-x \Theta}\right)\right]^{1 / 2},
\end{aligned}
$$

which one can use for further computation.

In fig. (2), we have depicted the model independent inflationary constraint from the primordial gravitational waves via tensor-to-scalar ratio on the dark matter relic abundance within the framework of effective field theory of RSII membrane paradigm. The yellow shaded region signifies the excluded region from Planck 2015+WMAP9 and Planck 2015+BICEP2/Keck Array joint data sets. The purple and black vertical lines signify the upper bound on the inflationary tensor-to-scalar ratio $r=0.12$ and $r=0.11$ obtained from Planck 2015+WMAP9 and Planck 2015+BICEP2/Keck Array joint data sets respectively. Also the grey coloured horizontal line signifies the bound on dark matter relic abundance $\Omega_{D M} h^{2}=0.1199 \pm 0.0027$, as obtained from Planck 2015 data. The red and blue coloured curve represents the behaviour of the dark matter relic abundance with respected tensor-to-scalar ratio in RSII membrane paradigm for $\langle\sigma v\rangle=1.5 \times 10^{-27} \mathrm{~cm}^{3} / \mathrm{s}$ and $\langle\sigma v\rangle=1.2 \times 10^{-27} \mathrm{~cm}^{3} / \mathrm{s}$ respectively. Here for both of the cases we fix the value of the dimensionless membrane parameter $\alpha=V_{\text {inf }} / \sigma=1.77 \times 10^{64}$. From fig. (2), it is clearly observed that for $r<0.01$, various inflationary models in membrane paradigm are in huge tension with the Planck 2015 constraint on dark matter relic abundance. This implies that the low scale inflationary models within RSII membrane paradigm are highly disfavoured. On the other hand, for $0.01 \leq r \leq 0.12$, various inflationary models in membrane paradigm are consistent with $2 \sigma$ constraint on dark matter relic abundance obtained from Planck 2015 data. This implies that the high scale inflationary models within RSII membrane paradigm are favoured for $0.01 \leq r \leq 0.12$.

In fig. (4(a)) and fig. (4(b)), we have depicted the model independent inflationary constraint from the primordial gravitational waves via tensor-to-scalar ratio on the thermally averaged annihilation cross-section of dark matter content for the dark matter mass, $m_{\chi}=100 \mathrm{GeV}$ and $m_{\chi}=1 \mathrm{TeV}$ respectively, within the framework of effective field theory of RSII membrane paradigm. For all of these three cases from the fig. (4(a)) and fig. (4(b)), it is clearly observed that the value of the inflationary tensor-to-scalar ratio decreases with the increase in the thermally averaged annihilation cross-section of dark matter content. Also it is important to note that for of these three cases within the allowed range of tensor-to-scalar ratio, $0.01 \leq r \leq 0.12$, as constrained from fig. (2) within RSII membrane paradigm, thermally averaged annihilation cross-section of dark matter content is constrained within the window, $\langle\sigma v\rangle \sim \mathcal{O}\left(10^{-28}-10^{-27}\right) \mathrm{cm}^{3} / \mathrm{s}$, which we will throughly follow in the rest of our analysis in this paper. 
In the next section, we will explicitly study the various constraints and consequences from the effective field theory of dark matter within the framework of RSII membrane paradigm.

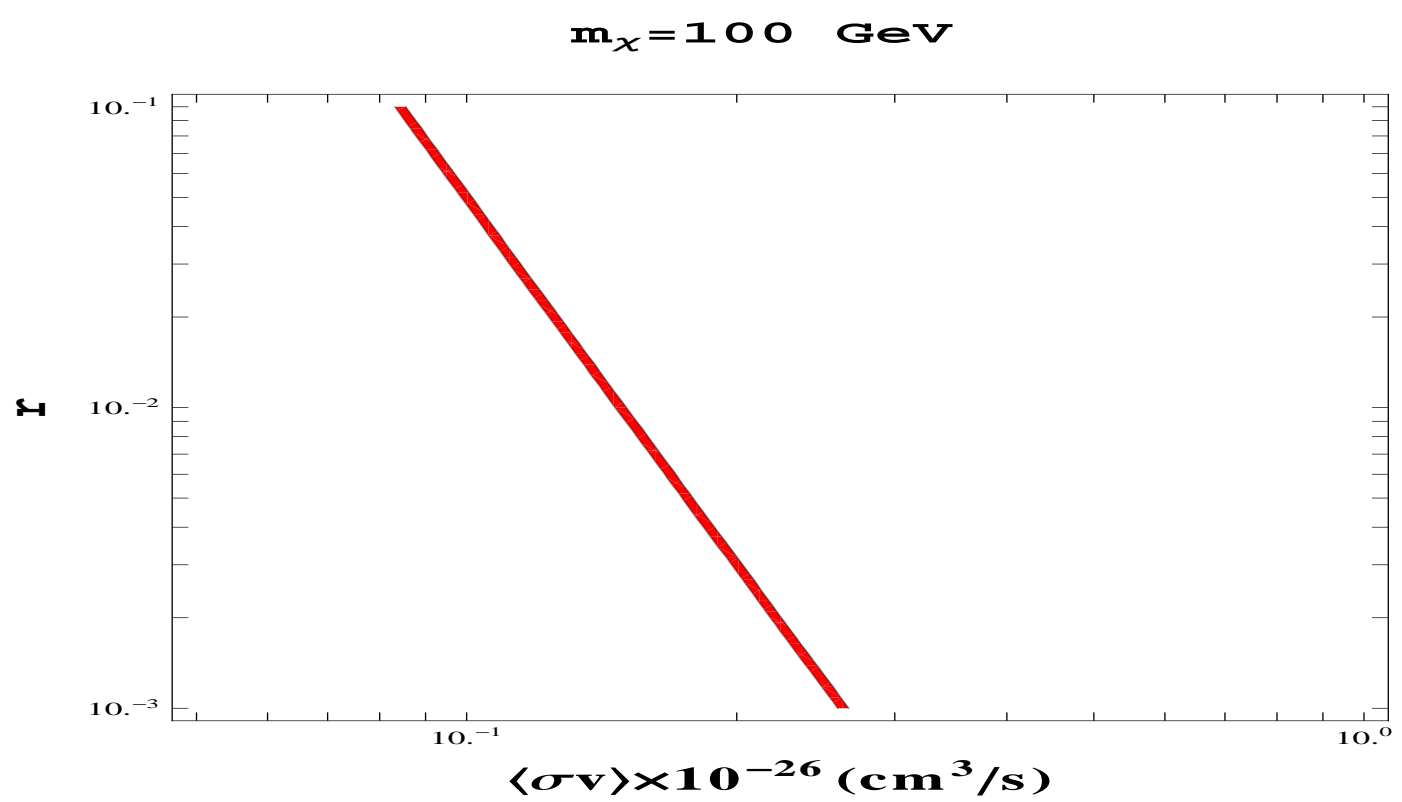

(a) $r$ vs $\langle\sigma v\rangle$ for $m_{\chi}=100 \mathrm{GeV}$.

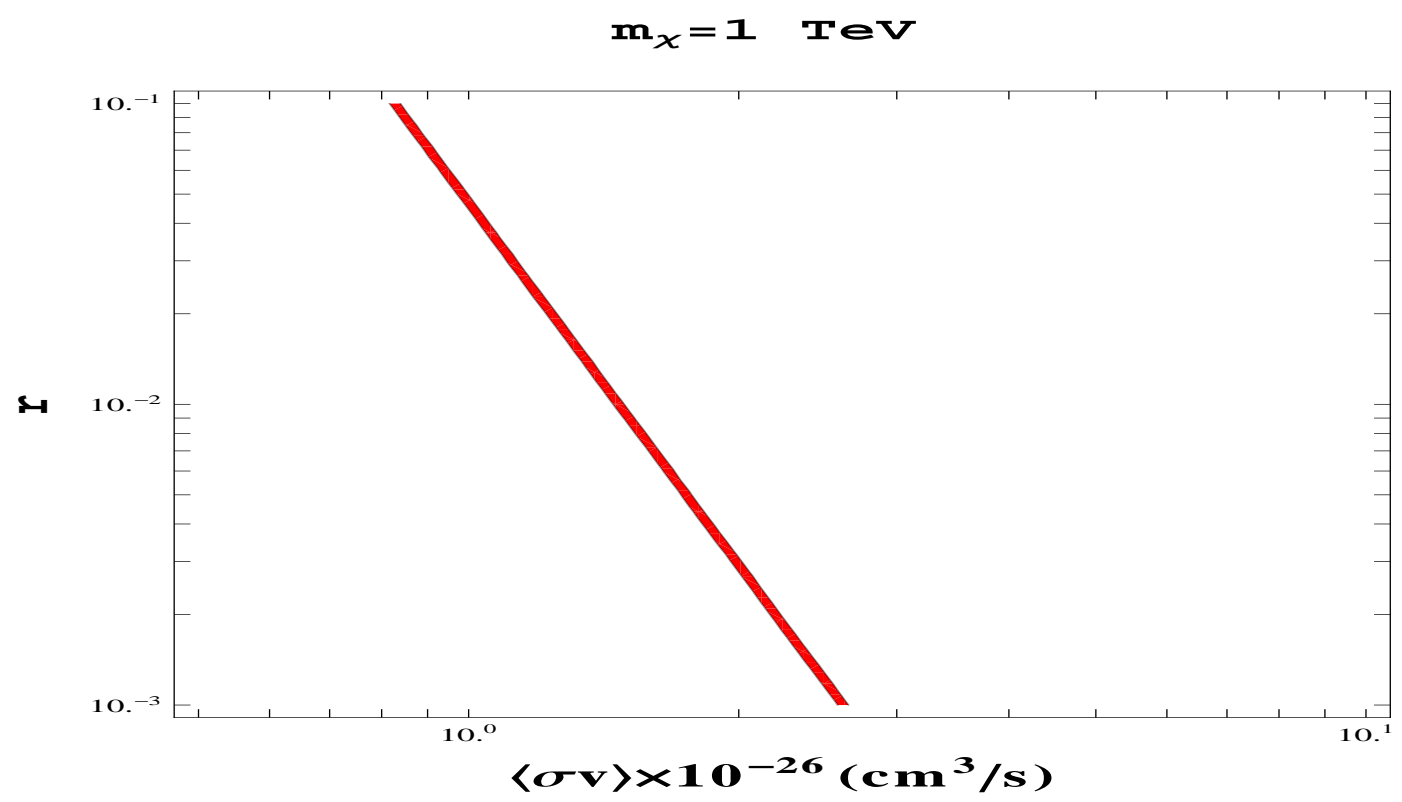

(b) $r$ vs $\langle\sigma v\rangle$ for $m_{\chi}=1 \mathrm{TeV}$.

Figure 4. In the above figure we have shown the allowed region of $\alpha$ with respect to the thermally averaged cross-section $\langle\sigma v\rangle$. 


\section{Effective Field Theory of dark matter from membrane paradigm}

In the next couple of subsections we will explicitly study the role of the characteristic parameter $\alpha$ of RSII membrane paradigm which effects the allowed parameter space of effective couplings in the membrane and the mediator mass in variants of effective field theory model. More more details effective theory see refs. [55]. For the sake of simplicity, in the present discussion we will assume that the couplings of dark matter with mediator and the mediator to fermions to be the same and we will follow this throughout the rest of the analysis performed in this paper. In table (1) we have explicitly mentioned the various types of dark matter, corresponding effective field theory operators, mediator spin and the coupling with mediator suppression scale.

\subsection{Dirac dark matter: spin-0 mediator}

\subsection{1 s-channel analysis}

To start with let us consider the following localized interactions for Dirac dark matter, $\chi$ and a spin-0 mediator, $A$, within the framework of effective field theory written in RSII membrane as:

$$
\mathcal{L}_{\text {brane }} \supset\left[\bar{\chi}\left(\lambda_{\chi_{S}}+\lambda_{\chi_{p}} i \gamma^{5}\right) \chi+\bar{f}\left(\lambda_{f_{S}}+\lambda_{f_{p}} i \gamma^{5}\right) f\right] A,
$$

where $\lambda_{\chi_{S}}, \lambda_{\chi_{p}}$ and $\lambda_{f_{S}}, \lambda_{f_{p}}$ are the fermionic couplings for dark matter $\chi$ and standard model (SM) fermion $f$. In fig. (8(a)) and fig. (8(b)), we have explicitly shown the Feynman diagrammatic representation of possible s-channel and t/u-channel processes for dirac dark matter with spin-0 mediator. The cross-section from the above Lagrangian after taking all the coupling equal is given as:

$$
\begin{aligned}
\sigma & =\frac{1}{8 \pi\left(s-4 m_{\chi}^{2}\right)} \int_{t_{-}}^{t_{+}}|\mathcal{M}|^{2} d t \\
& =\frac{n_{c} g^{4}}{8 \pi s\left(\left(s-m_{A}^{2}\right)^{2}+m_{A}^{2} \Gamma_{A}^{2}\right)} \sqrt{\left[\frac{1-4 m_{f}^{2} / s}{1-4 m_{\chi}^{2} / s}\right]}\left(s-2 m_{f}^{2}\right)\left(s-2 m_{\chi}^{2}\right) .
\end{aligned}
$$

where $n_{c}=3$ for quarks and 1 for leptons, $g_{A}$ and $m_{A}$ are the respective coupling and the mass of the mediator. The matrix element for the S-matrix and the symbol $t_{ \pm}$is given by:

$$
\begin{aligned}
|\mathcal{M}|^{2} & =\frac{\left(s-2 m_{\chi}^{2}\right)\left(s-2 m_{f}^{2}\right)}{\left(s-m_{A}^{2}\right)^{2}+m_{A}^{2} \Gamma_{A}} \\
t_{ \pm} & =\left(m_{\chi}^{2}+m_{f}^{2}-\frac{s}{2}\right) \pm \frac{\sqrt{\left(s-4 m_{\chi}^{2}\right)\left(s-4 m_{f}^{2}\right)}}{2} .
\end{aligned}
$$




\begin{tabular}{c|c|c|c|}
\hline \hline Type of dark matter & Effective Operator & mediator spin & coupling with \\
mediator \\
\hline \hline Dirac Dark Matter & $\bar{\chi} \chi \bar{f} f$ & spin-0 & $\frac{g^{2}}{M^{2}}$ \\
Majorana Dark Matter & $\bar{\chi} \chi \bar{f} f$ & spin-0 & $\frac{g^{2}}{M^{2}}$ \\
Dirac Dark Matter & $\bar{\chi} \gamma^{\mu} \chi \bar{f} \gamma_{\mu} f$ & spin-1 & $\frac{g^{2}}{M^{2}}$ \\
Majorana Dark Matter & $\bar{\chi} \gamma^{\mu} \gamma_{5} \chi \bar{f} \gamma_{\mu} f$ & spin-1 & $\frac{g^{2}}{M^{2}}$ \\
Complex Scalar Dark Matter & $\phi^{*} \phi \bar{f} f$ & spin-0 & $\frac{|\mu|^{2}}{M^{2}}$ \\
Real Scalar Dark Matter & $\phi^{2} \bar{f} f$ & spin-0 & $\frac{\mu^{2}}{M^{2}}$ \\
Complex Scalar Dark Matter & $\phi^{*} \partial_{\mu} \phi \bar{f} \gamma^{\mu} f$ & spin-1 & $\frac{g^{2}}{M^{2}}$ \\
Real Scalar Dark Matter & $\phi \partial_{\mu} \phi \bar{f} \gamma^{\mu} f$ & spin-1 & $\frac{g^{2}}{M^{2}}$ \\
Real Vector Scalar Dark Matter & $X^{\mu} X_{\mu}^{*} \bar{f} f$ & spin-0 & $\frac{\left|\mu_{X}\right| g}{M^{2}}$ \\
Complex Vector Scalar Dark Matter & $X^{* \nu} \partial_{\nu} X_{\mu} \bar{f} \gamma^{\mu} f$ & $\frac{\mu_{X} \mid g}{M^{2}}$ \\
\hline \hline \\
\hline \hline
\end{tabular}

Table 1. Tabular representation of various types of dark matter, corresponding effective field theory operators, mediator spin and the coupling with mediator suppression scale.

For a low velocity perturbation regime by taking the following approximation:

$$
s=4 m_{\chi}^{2}\left(1+v^{2} / 4\right)
$$

we finally get the following simplified expression for the product of annihilation crosssection and velocity as:

$$
\sigma v \simeq a+\mathcal{O}\left(v^{2}\right)
$$




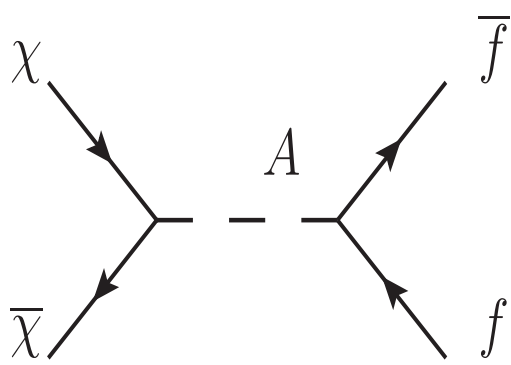

(a) $\chi \bar{\chi} \rightarrow f \bar{f}$ process for s-channel.

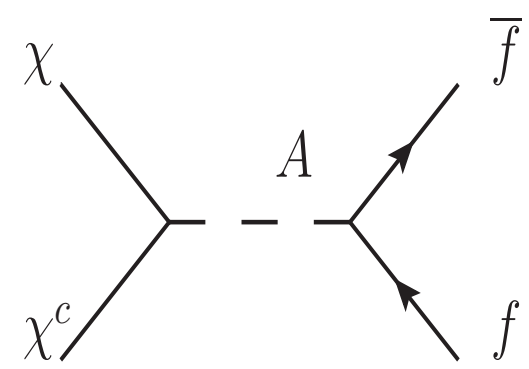

(b) $\chi \chi^{c} \rightarrow f \bar{f}$ process for s-channel.

Figure 5. Feynman diagrammatic representation of s-channel channel processes for dirac dark matter and Majorana with spin-0 mediator.

where in the present context the factor $a$ is given by:

$$
a=n_{c} g^{2} \frac{2 m_{\chi}^{2}-m_{f}^{2}}{2 \pi\left(m^{4}-8 m^{2} m_{\chi}^{2}+16 m_{\chi}^{4}+m^{2} \Gamma^{2}\right)} \sqrt{1-\frac{m_{f}^{2}}{m_{\chi}^{2}}}
$$

and the mediator's width to SM fermions is given by:

$$
\Gamma=\sum_{f} \Gamma(A \rightarrow f \bar{f})=\frac{g^{4} n_{c} m}{4 \pi}\left[1-4 \frac{m_{f}^{2}}{m^{2}}\right]^{1 / 2}\left[1-2 \frac{m_{f}^{2}}{m^{2}}\right] .
$$

The relic abundance is given as:

$$
\Omega_{D M} h^{2}=\frac{1.07 \times 10^{9}}{J\left(x_{f}\right) g_{*}^{1 / 2} M_{p}}
$$

where the $J\left(x_{f}\right)$ for this spin-0 mediator s-channel process is given by:

$$
J\left(x_{f}\right)=\int_{x_{f}}^{\infty} n_{c} g^{2} \frac{2 m_{\chi}^{2}-m_{f}^{2}}{2 \pi\left(m^{4}-8 m^{2} m_{\chi}^{2}+16 m_{\chi}^{4}+m^{2} \Gamma^{2}\right) x^{2} f_{\text {membrane }}(x)} \sqrt{1-\frac{m_{f}^{2}}{m_{\chi}^{2}}} d x
$$

where the function $f_{\text {membrane }}(x)$ is the characteristic parameter for RS single braneworld and can be expressed in terms of tensor-to-scalar ratio $(r)$ which is given in Eq.(3.29). Now for the GR limiting case of the $f_{\text {membrane }}(x) \rightarrow 1$ and then the relic abundance will only depend on the mass of the Dark Matter $\left(m_{\chi}\right), g_{A}$ the coupling with the spin-0 mediator and the mass of the mediator $\left(m_{A}\right)$.

$$
\Omega_{D M} h^{2}=\frac{1.07 \times 10^{9} x_{f}}{g_{*}^{1 / 2} M_{p}}\left(n_{c} g^{2} \frac{2 m_{\chi}^{2}-m_{f}^{2}}{2 \pi\left(m^{4}-8 m^{2} m_{\chi}^{2}+16 m_{\chi}^{4}+m^{2} \Gamma^{2}\right)} \sqrt{1-\frac{m_{f}^{2}}{m_{\chi}^{2}}}\right)^{-1}
$$




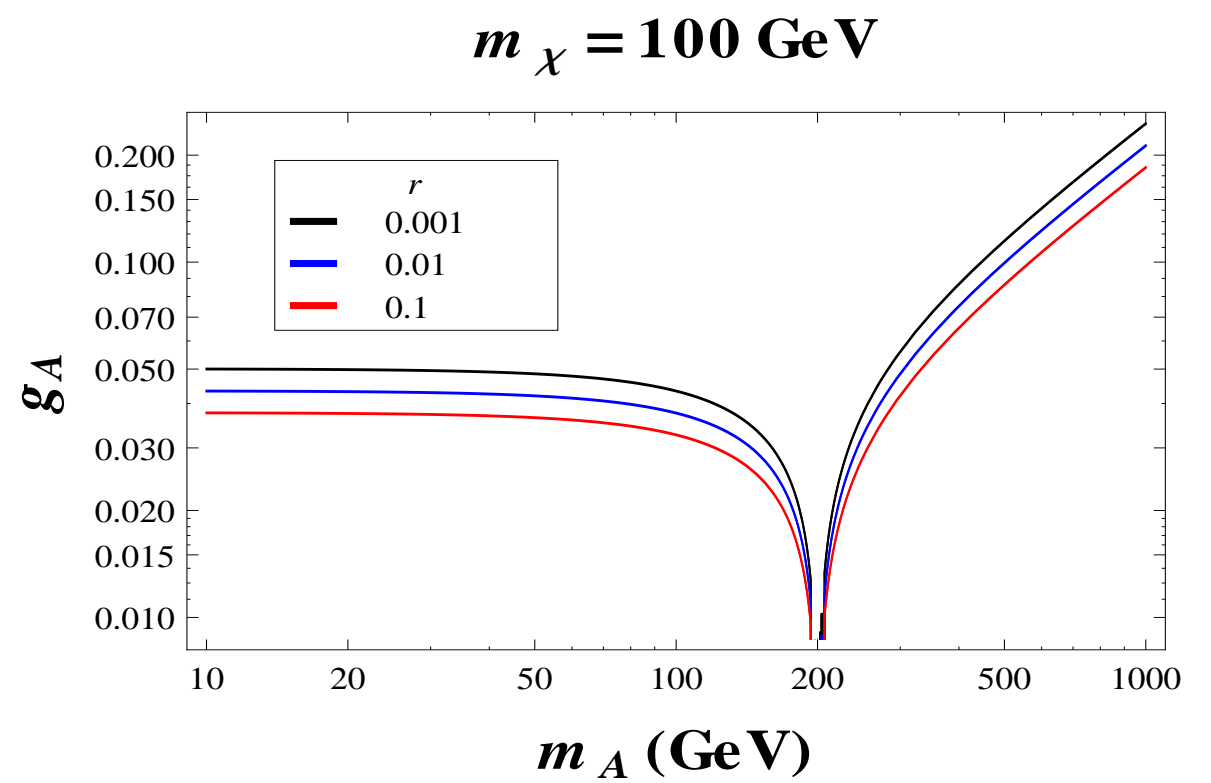

(a) $g_{A}$ vs $m_{A}$ for s-channel with $m_{\chi}=100 \mathrm{GeV}$.

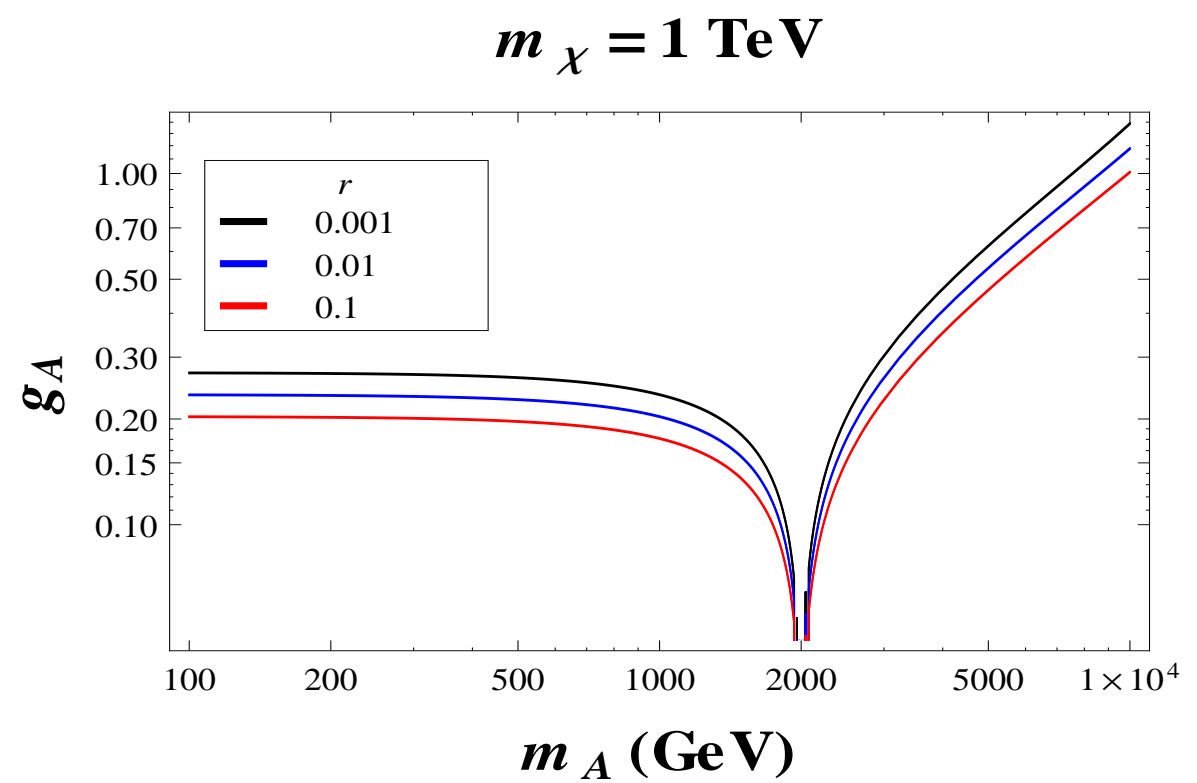

(b) $g_{A}$ vs $m_{A}$ for s-channel with $m_{\chi}=1 \mathrm{TeV}$.

Figure 6. In the above figure we have shown the allowed region of $g_{A}$ with respect to the mass of the mediator $m_{A}$, for the s-channel processes.

In order to constrain the coupling $\left(g_{A}\right)$ and mass $\left(m_{A}\right)$ we take the present data of the relic abundance $\left(\Omega_{D M} h^{2}=0.1199 \pm 0.0027[56]\right)$ and constrain the function $J\left(x_{f}\right)$, which in turn constrain the coupling $g_{A}$ and $m_{A}$ for a particular tensor-to-scalar ratio $(r)$. We have not shown the GR limiting case as it has been extensively been explored in [55]. In fig. (6(a)) and fig. (6(b)), we have depicted the behaviour of the effective 
coupling of spin-0 mediator $g_{A}$ with the varying mass the spin-0 mediator $m_{A}$ for s-channel process with three distinct value of the tensor-to-scalar ratio $r=0.001$, $r=0.01$ and $r=0.1$ respectively in RSII membrane. We also consider two different values of the dark matter mass $m_{\chi}=100 \mathrm{GeV}$ and $m_{\chi}=1 \mathrm{TeV}$ for the s-channel analysis. From fig. (6(a)) and fig. (6(b)), it is clearly observed that the behaviour of the effective coupling of spin- 0 mediator $g_{A}$ with the varying mass the spin- 0 mediator $m_{A}$ are similar in both of the cases and also sensitive in the vicinity of $m_{A}=2 \times 10^{2} \mathrm{GeV}$ and $m_{A}=2 \times 10^{3} \mathrm{GeV}$ respectively as it has a resonance (i.e $2 m_{X}=m_{A}$ ). Most importantly, in both the sides of $m_{A}=2 \times 10^{2} \mathrm{GeV}$ and $m_{A}=2 \times 10^{3} \mathrm{GeV}$ the coupling of spin- 0 mediator $g_{A}$ behave in completely opposite manner.

\subsection{2 $\mathrm{t} / \mathrm{u}$ - channel analysis}

Next we consider the following localized interactions for Dirac dark matter, $\chi$ and a spin-0 mediator, $A$, within the framework of effective field theory written in RSII membrane as:

$$
\mathcal{L}_{\text {membrane }} \supset \bar{\chi}\left(\lambda_{\chi_{S}}+\lambda_{\chi_{p}} i \gamma^{5}\right) f A+\bar{f}\left(\lambda_{f_{S}}+\lambda_{f_{p}} i \gamma^{5}\right) \chi A^{\dagger}
$$

Now, considering all the couplings to be of the same order the thermally averaged cross-section becomes: $\lambda_{f_{S}} \sim \lambda_{f_{p}} \sim \lambda_{\chi_{S}} \sim \lambda_{\chi_{p}} \sim g_{A}$ the amplitude is given as

$$
\sigma=\frac{1}{8 \pi\left(s-4 m_{\chi}^{2}\right)} \int_{t_{-}}^{t_{+}}|\mathcal{M}|^{2} d t
$$

where the matrix element for the S-matrix and $t_{ \pm}$is defined as:

$$
\begin{aligned}
|\mathcal{M}|^{2} & =g_{A}^{4} n_{c} \frac{\left(m_{f}^{2}+m_{\chi}^{2}-t\right)^{2}}{\left(t-m_{A}^{2}\right)^{2}} \\
t_{ \pm} & =\left(m_{\chi}^{2}+m_{f}^{2}-\frac{s}{2}\right) \pm \frac{\sqrt{\left(s-4 m_{\chi}^{2}\right)\left(s-4 m_{f}^{2}\right)}}{2} .
\end{aligned}
$$

where $n_{c}=3$ for quarks and 1 for leptons, $g_{A}$ and $m_{A}$ are the respective coupling and the mass of the mediator. Now taking the following approximation:

$$
s=4 m_{\chi}^{2} /\left(1-v^{2} / 4\right)
$$

we finally get the following simplified expression for the product of annihilation crosssection and velocity as:

$$
\sigma v=a+\mathcal{O}\left(v^{2}\right)
$$

where in the present context, the factor $a$ is given by:

$$
a \approx \frac{n_{c} g^{4} \sqrt{1-m_{f}^{2} / m_{\chi}^{2}}}{4 \pi\left(m^{2}-m_{f}^{2}+m_{\chi}^{2}\right)^{2}} m_{\chi}^{2}
$$




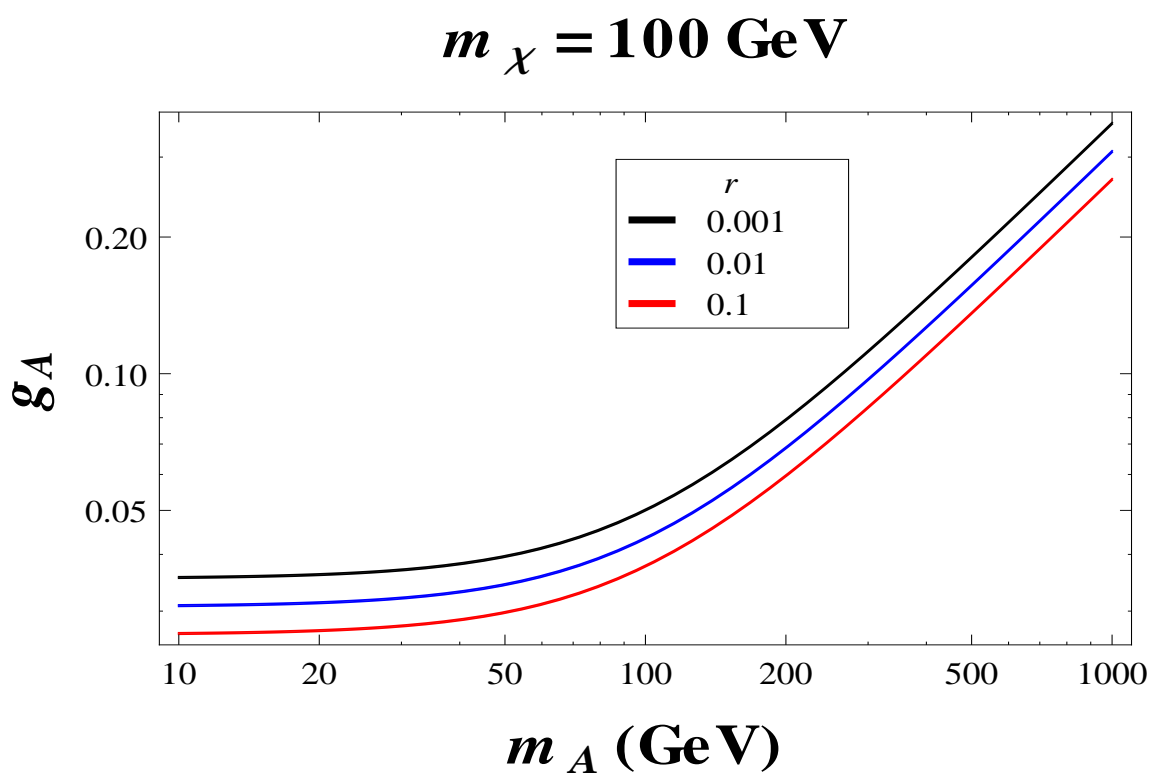

(a) $g_{A}$ vs $m_{A}$ for t/u-channel with $m_{\chi}=100 \mathrm{GeV}$.

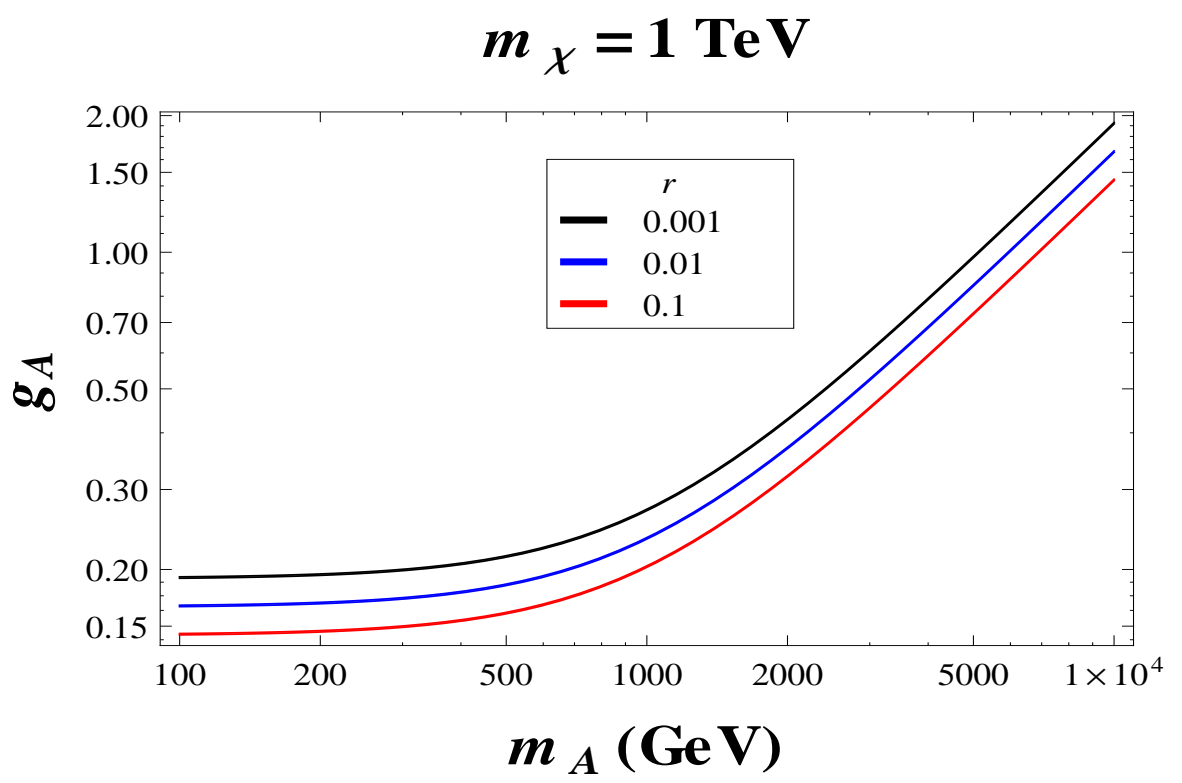

(b) $g_{A}$ vs $m_{A}$ for t/u-channel with $m_{\chi}=1 \mathrm{TeV}$.

Figure 7. In the above figure we have shown the allowed region of $g_{A}$ with respect to the mass of the mediator $m_{A}$, for the $\mathrm{t} / \mathrm{u}$ - channel processes.

The dark matter relic abundance in this case is given as:

$$
\Omega_{D M} h^{2}=\frac{1.07 \times 10^{9}}{J\left(x_{f}\right) g_{*}^{1 / 2} M_{p}}
$$




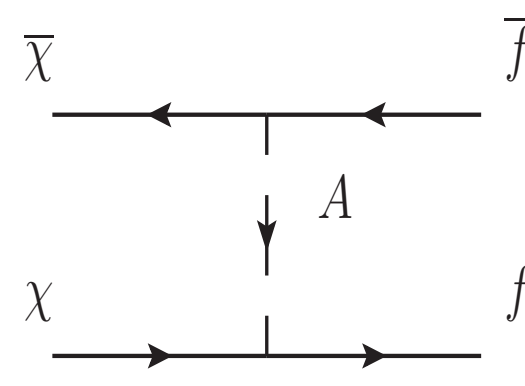

(a) $\chi \bar{\chi} \rightarrow f \bar{f}$ process for $\mathrm{t} / \mathrm{u}$-channel.

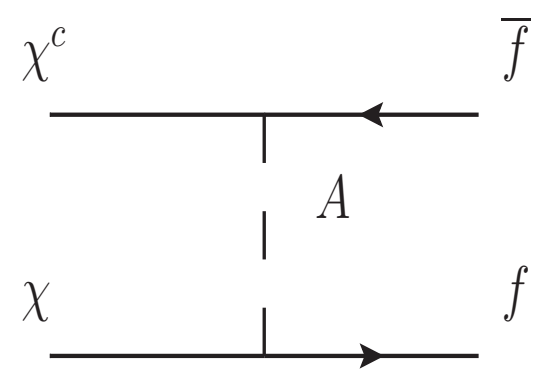

(b) $\chi \chi^{c} \rightarrow f \bar{f}$ process for $\mathrm{t} / \mathrm{u}$-channel.

Figure 8. Feynman diagrammatic representation of $\mathrm{t} / \mathrm{u}$-channel channel processes for Dirac dark matter and Majorana with spin-0 mediator.

where $J\left(x_{f}\right)$ for spin-0 mediator in $\mathrm{t} / \mathrm{u}$ channel is given by:

$$
J\left(x_{f}\right)=\int_{x_{f}}^{\infty} \frac{n_{c} g^{4} \sqrt{1-m_{f}^{2} / m_{\chi}^{2}} m_{\chi}^{2}}{4 \pi\left(m^{2}-m_{f}^{2}+m_{\chi}^{2}\right)^{2} x^{2} f_{\text {membrane }}(x)} d x
$$

where the function $f_{\text {membrane }}(x)$ is the characteristic parameter for RS single braneworld and this can be expressed in terms of tensor-to-scalar ratio $(r)$ which is given in Eq.(3.29). Now for the GR limiting case of the $f_{\text {membrane }}(x) \rightarrow 1$ and then the relic abundance will only depend on the mass of the Dark Matter $\left(m_{\chi}\right), g_{A}$ the coupling with the spin- 0 mediator and the mass of the mediator $\left(m_{A}\right)$.

$$
\Omega_{D M} h^{2}=\frac{1.07 \times 10^{9} x_{f}}{g_{*}^{1 / 2} M_{p}}\left(\frac{n_{c} g^{4} \sqrt{1-m_{f}^{2} / m_{\chi}^{2}}}{4 \pi\left(m^{2}-m_{f}^{2}+m_{\chi}^{2}\right)^{2}} m_{\chi}^{2}\right)^{-1}
$$

In order to constrain the coupling $\left(g_{A}\right)$ and mass $\left(m_{A}\right)$ we take the present data of the relic abundance $\left(\Omega_{D M} h^{2}=0.1199 \pm 0.0027[56]\right)$ and constrain the function $J\left(x_{f}\right)$, which in turn constrain the coupling $g_{A}$ and $m_{A}$ for a particular tensor-to-scalar ratio $(r)$. We have not shown the GR limiting case as it has been extensively been explored in [55]. In fig. (7(a)) and fig. (7(b)), we have depicted the behaviour of the effective coupling of spin- 0 mediator $g_{A}$ with the varying mass the spin-0 mediator $m_{A}$ for $\mathrm{t} / \mathrm{u}$-channel process with three distinct value of the tensor-to-scalar ratio $r=0.001, r=0.01$ and $r=0.1$ respectively in RSII membrane. We also consider two different values of the dark matter mass $m_{\chi}=100 \mathrm{GeV}$ and $m_{\chi}=1 \mathrm{TeV}$ for the t/u-channel analysis. From fig. (7(a)) and fig. (7(b)), it is clearly observed that the behaviour of the effective coupling of spin-0 mediator $g_{A}$ with the varying mass the spin-0 mediator $m_{A}$ are similar for both of the cases and also sensitive in the vicinity of $m_{A}=1 \times 10^{2} \mathrm{GeV}$ and $m_{A}=1 \times 10^{3} \mathrm{GeV}$ respectively as it has a resonance (i.e $2 m_{X}=m_{A}$ ). Most importantly, in both the sides of $m_{A}=1 \times 10^{2} \mathrm{GeV}$ and 
$m_{A}=1 \times 10^{3} \mathrm{GeV}$ the coupling of spin- 0 mediator $g_{A}$ behave in completely opposite manner.

\subsection{Dirac Dark matter: spin-1 mediator}

\subsection{1 s-channel analysis}

Next we consider the following localized interactions for a Dirac dark matter particle, $\chi$, and a spin-1 mediator, $V_{\mu}$, within the framework of effective field theory written in RSII membrane as:

$$
\mathcal{L}_{\text {membrane }} \supset\left[\bar{\chi} \gamma^{\mu}\left(g_{\chi_{V}}+g_{\chi_{a}} \gamma_{5}\right) \chi+\bar{f} \gamma^{\mu}\left(g_{f_{V}}+g_{f_{a}}\right) f\right] V_{\mu}
$$

In fig. (9(a)), fig. (9(b)), fig. (10(a)) and fig. (10(b)), we have explicitly shown the Feynman diagrammatic representation of possible s-channel and t/u-channel processes for Dirac dark matter with spin-1 mediator respectively. After taking the

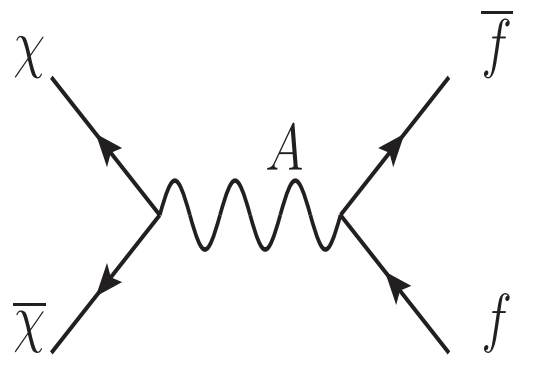

(a) $\chi \bar{\chi} \rightarrow f \bar{f}$ process for s-channel.

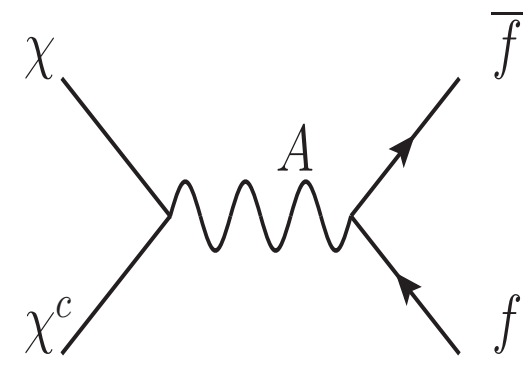

(b) $\chi \chi^{c} \rightarrow f \bar{f}$ process for s-channel.

Figure 9. Feynman diagrammatic representation of s-channel processes for dirac dark matter with spin-1 mediator.

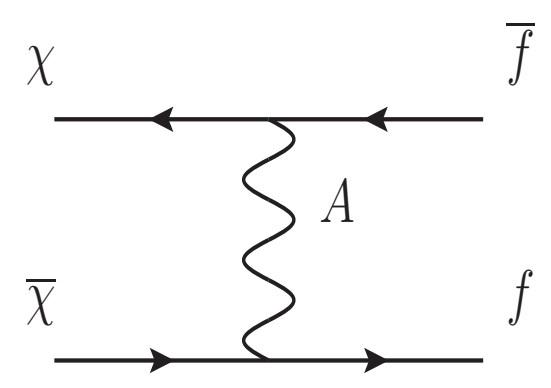

(a) $\chi \bar{\chi} \rightarrow f \bar{f}$ process for $\mathrm{t} / \mathrm{u}$-channel.

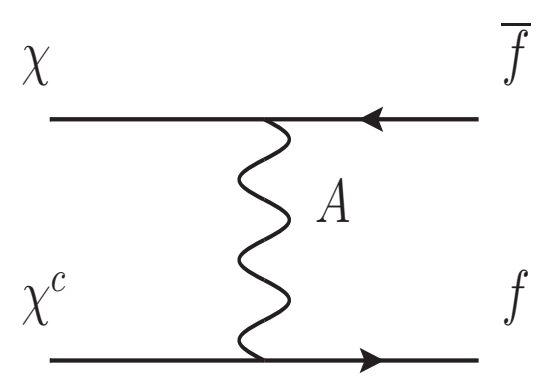

(b) $\chi \chi^{c} \rightarrow f \bar{f}$ process for $\mathrm{t} / \mathrm{u}$-channel.

Figure 10. Feynman diagrammatic representation of $\mathrm{t} / \mathrm{u}$-channel processes for Dirac dark matter with spin-1 mediator. 
equal coupling approximation the cross-section from the above Lagrangian is computed as:

$$
\sigma=\frac{1}{8 \pi\left(s-4 m_{\chi}^{2}\right)} \int_{t_{-}}^{t_{+}}|\mathcal{M}|^{2} d t
$$

where the matrix element for the S-matrix and the symbol $t_{ \pm}$is given by:

$$
\begin{aligned}
|\mathcal{M}|^{2} & =\frac{2 n_{c} g^{4}}{3 \pi s\left(\left(s-m_{A}^{2}\right)^{2}+m^{2} \Gamma^{2}\right)}\left\{4 m_{\chi}^{2} m_{f}^{2}\left(7-6 \frac{s}{m^{2}}+\frac{3 s^{2}}{m^{4}}\right)-4 m_{\chi}^{2} s\right. \\
& \left.+s\left(s-4 m_{f}^{2}\right)+\left(s-4 m_{f}^{2}\right)\left(s+2 m_{\chi}^{2}\right)+2\left(s+2 m_{f}^{2}\right)\left(s-m_{\chi}^{2}\right)\right\} \\
t_{ \pm} & =\left(m_{\chi}^{2}+m_{f}^{2}-\frac{s}{2}\right) \pm \frac{\sqrt{\left(s-4 m_{\chi}^{2}\right)\left(s-4 m_{f}^{2}\right)}}{2}
\end{aligned}
$$

where $n_{c}=3$ for quarks and 1 for leptons, $g_{A}$ and $m_{A}$ are the respective coupling and the mass of the mediator. For a low velocity perturbation by taking the following approximation:

$$
s=4 m_{\chi}^{2}\left(1+v^{2} / 4\right)
$$

we finally get the following simplified expression for the product of annihilation crosssection and velocity as:

$$
\sigma v \simeq a+\mathcal{O}\left(v^{2}\right)
$$

where in the present context, the factor $a$ is given by:

$$
a=2 n_{c} g^{4} \frac{m_{\chi}^{2}}{\pi m^{4}} \sqrt{1-\frac{m_{f}^{2}}{m_{\chi}^{2}}} \frac{1-2 \frac{m_{f}^{2}}{m^{2}}+4 \frac{m_{\chi}^{2} m_{f}^{2}}{m^{4}}}{2 \pi\left(1-8 \frac{m_{\chi}^{2}}{m^{2}}+16 \frac{m_{\chi}^{4}}{m^{4}}+\frac{\Gamma^{2}}{m^{2}}\right)}
$$

and the mediator's width to SM fermions is given by:

$$
\Gamma=\sum_{f} \Gamma(A \rightarrow f \bar{f})=\frac{g^{2} n_{c} m}{6 \pi}\left[1-4 \frac{m_{f}^{2}}{m^{2}}\right]^{1 / 2}\left[1-2 \frac{m_{f}^{2}}{m^{2}}\right]
$$

The drak matter relic abundance in this case is given as:

$$
\Omega_{D M} h^{2}=\frac{1.07 \times 10^{9}}{J\left(x_{f}\right) g_{*}^{1 / 2} M_{p}}
$$

where $J\left(x_{f}\right)$ for spin-1 mediator s-channel is give by:

$$
J\left(x_{f}\right)=\int_{x_{f}}^{\infty} 2 n_{c} g^{4} \frac{m_{\chi}^{2}}{\pi m^{4}} \sqrt{1-\frac{m_{f}^{2}}{m_{\chi}^{2}}} \frac{1-2 \frac{m_{f}^{2}}{m^{2}}+4 \frac{m_{\chi}^{2} m_{f}^{2}}{m^{4}}}{2 \pi\left(1-8 \frac{m_{\chi}^{2}}{m^{2}}+16 \frac{m_{\chi}^{4}}{m^{4}}+\frac{\Gamma^{2}}{m^{2}}\right)^{2} x^{2} f_{\text {membrane }}(x)} d x
$$


where the function $f_{\text {membrane }}(x)$ is the characteristic parameter for RS single braneworld and this can be expressed in terms of tensor-to-scalar ratio $(r)$ which is given in Eq.(3.29). Now for the GR limiting case of the $f_{\text {membrane }}(x) \rightarrow 1$ and then the relic abundance will only depend on the mass of the Dark Matter $\left(m_{\chi}\right), g_{A}$ the coupling with the spin-0 mediator and the mass of the mediator $\left(m_{A}\right)$.

$$
\Omega_{D M} h^{2}=\frac{1.07 \times 10^{9} x_{f}}{g_{*}^{1 / 2} M_{p}}\left(2 n_{c} g^{4} \frac{m_{\chi}^{2}}{\pi m^{4}} \sqrt{1-\frac{m_{f}^{2}}{m_{\chi}^{2}}} \frac{1-2 \frac{m_{f}^{2}}{m^{2}}+4 \frac{m_{\chi}^{2} m_{f}^{2}}{m^{4}}}{2 \pi\left(1-8 \frac{m_{\chi}^{2}}{m^{2}}+16 \frac{m_{\chi}^{4}}{m^{4}}+\frac{\Gamma^{2}}{m^{2}}\right)}\right)^{-1} .
$$

In order to constrain the coupling $\left(g_{A}\right)$ and mass $\left(m_{A}\right)$ we take the present data of the relic abundance $\left(\Omega_{D M} h^{2}=0.1199 \pm 0.0027[56]\right)$ and constrain the function $J\left(x_{f}\right)$, which in turn constrain the coupling $g_{A}$ and $m_{A}$ for a particular tensor-to-scalar ratio $(r)$. We have not shown the GR limiting case as it has been extensively been explored in [55]. In fig. (11(a)) and fig. (11(b)), we have depicted the behaviour of the effective coupling of spin-1 mediator $g_{A}$ with the varying mass the spin-1 mediator $m_{A}$ for s-channel process with three distinct value of the tensor-to-scalar ratio $r=0.001$, $r=0.01$ and $r=0.1$ respectively in RSII membrane. We also consider two different values of the dark matter mass $m_{\chi}=100 \mathrm{GeV}$ and $m_{\chi}=1 \mathrm{TeV}$ for the s-channel analysis. From fig. (11(a)) and fig. (11(b)), it is clearly observed that the behaviour of the effective coupling of spin-1 mediator $g_{A}$ with the varying mass the spin-1 mediator $m_{A}$ are similar for both of the cases and also sensitive in the vicinity of $m_{A}=2 \times 10^{2} \mathrm{GeV}$ and $m_{A}=2 \times 10^{3} \mathrm{GeV}$ respectively as it has a resonance (i.e $2 m_{X}=m_{A}$ ). Most importantly, in both the sides of $m_{A}=2 \times 10^{2} \mathrm{GeV}$ and $m_{A}=2 \times 10^{3} \mathrm{GeV}$ the coupling of spin-1 mediator $g_{A}$ behave in completely opposite manner.

\subsection{2 $\mathrm{t} / \mathrm{u}$ - channel analysis}

Next we consider the following localized interactions for a Dirac dark matter particle, $\chi$, and a spin-1 mediator, $V_{\mu}$, within the framework of effective field theory written in RSII membrane as:

$$
\mathcal{L}_{\text {membrane }} \supset \bar{\chi} \gamma^{\mu}\left(g_{\chi_{V}}+g_{\chi_{a}} \gamma_{5}\right) f V_{\mu}+\bar{f} \gamma^{\mu}\left(g_{f_{V}}+g_{f_{a}}\right) \chi V_{\mu}^{\dagger}
$$

Now, taking all the couplings to be the same the cross-section becomes

$$
\sigma=\frac{1}{8 \pi\left(s-4 m_{\chi}^{2}\right)} \int_{t_{-}}^{t_{+}}|\mathcal{M}|^{2} d t
$$




\section{$m_{\chi}=100 \mathrm{GeV}$}

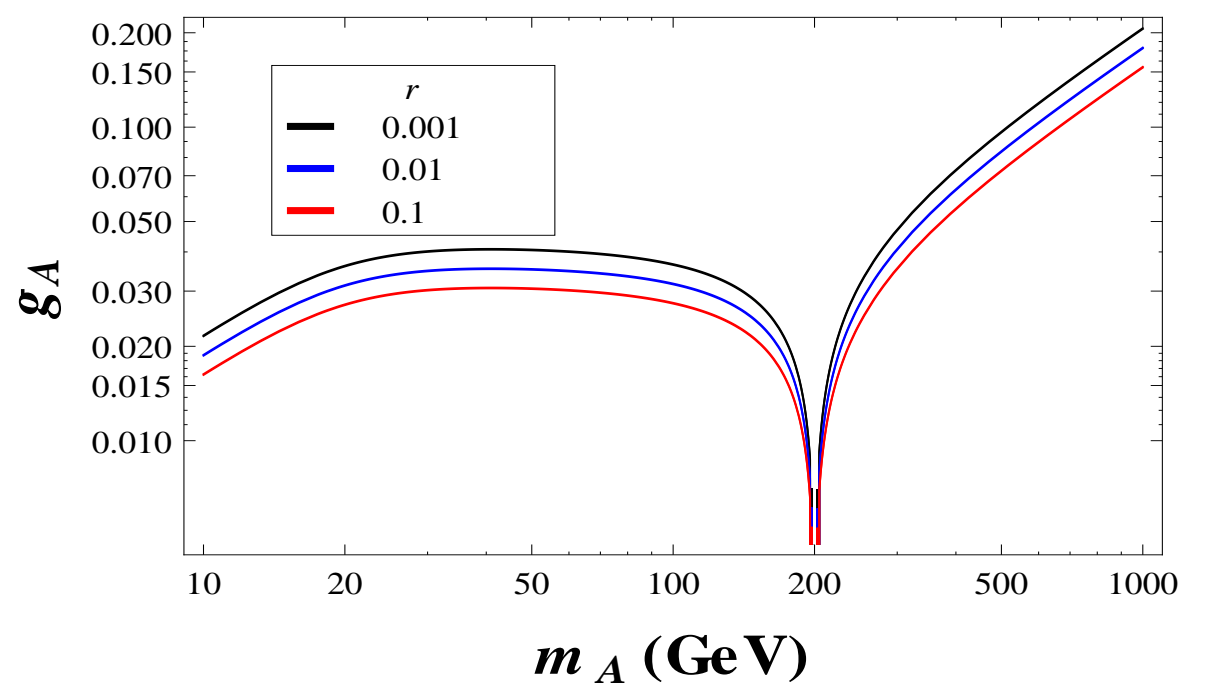

(a) $g_{A}$ vs $m_{A}$ for s-channel with $m_{\chi}=100 \mathrm{GeV}$.

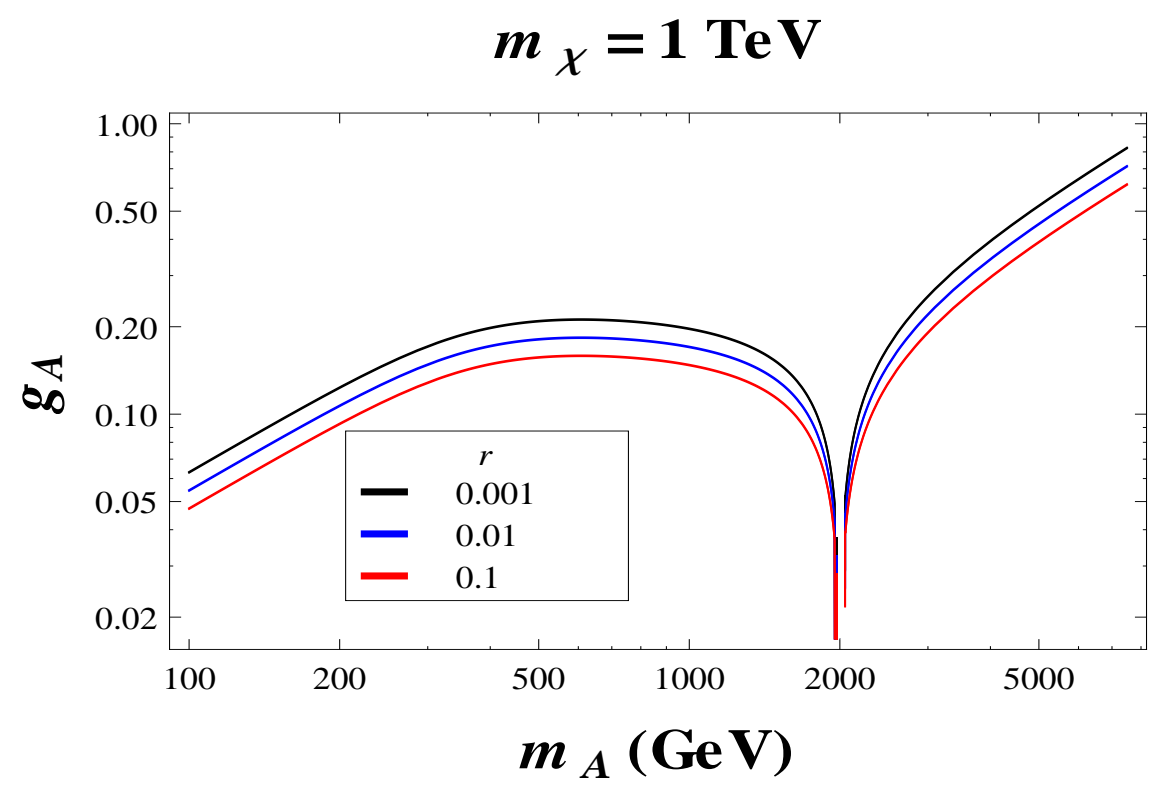

(b) $g_{A}$ vs $m_{A}$ for s-channel with $m_{\chi}=1 \mathrm{TeV}$.

Figure 11. In the above figure we have shown the allowed region of $g_{A}$ with respect to the mass of the mediator $m_{A}$, the upper panel is for the s-channel processes.

where the matrix element for the S-matrix and the symbol $t_{ \pm}$is given by:

$$
\begin{aligned}
|\mathcal{M}|^{2} & =\frac{g_{A}^{4} n_{c}}{4 \pi s\left(t-m_{A}^{2}\right)^{2}}\left(\left(s-2 m_{\chi}^{2}\right)\left(s-2 m_{f}^{2}\right)+\left(m_{\chi}^{2}+m_{f}^{2}-t\right)^{2}\right), \\
t_{ \pm} & =\left(m_{\chi}^{2}+m_{f}^{2}-\frac{s}{2}\right) \pm \frac{\sqrt{\left(s-4 m_{\chi}^{2}\right)\left(s-4 m_{f}^{2}\right)}}{2}
\end{aligned}
$$




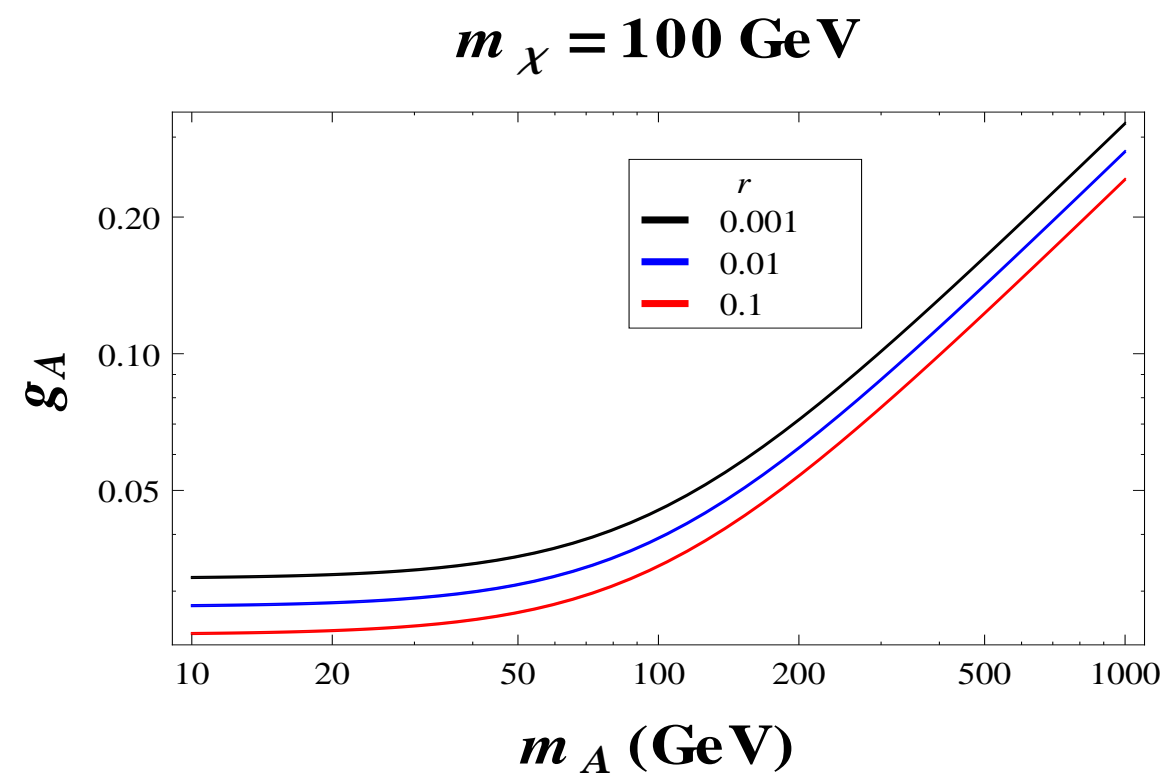

(a) $g_{A}$ vs $m_{A}$ for $\mathrm{t} / \mathrm{u}$-channel with $m_{\chi}=100 \mathrm{GeV}$.

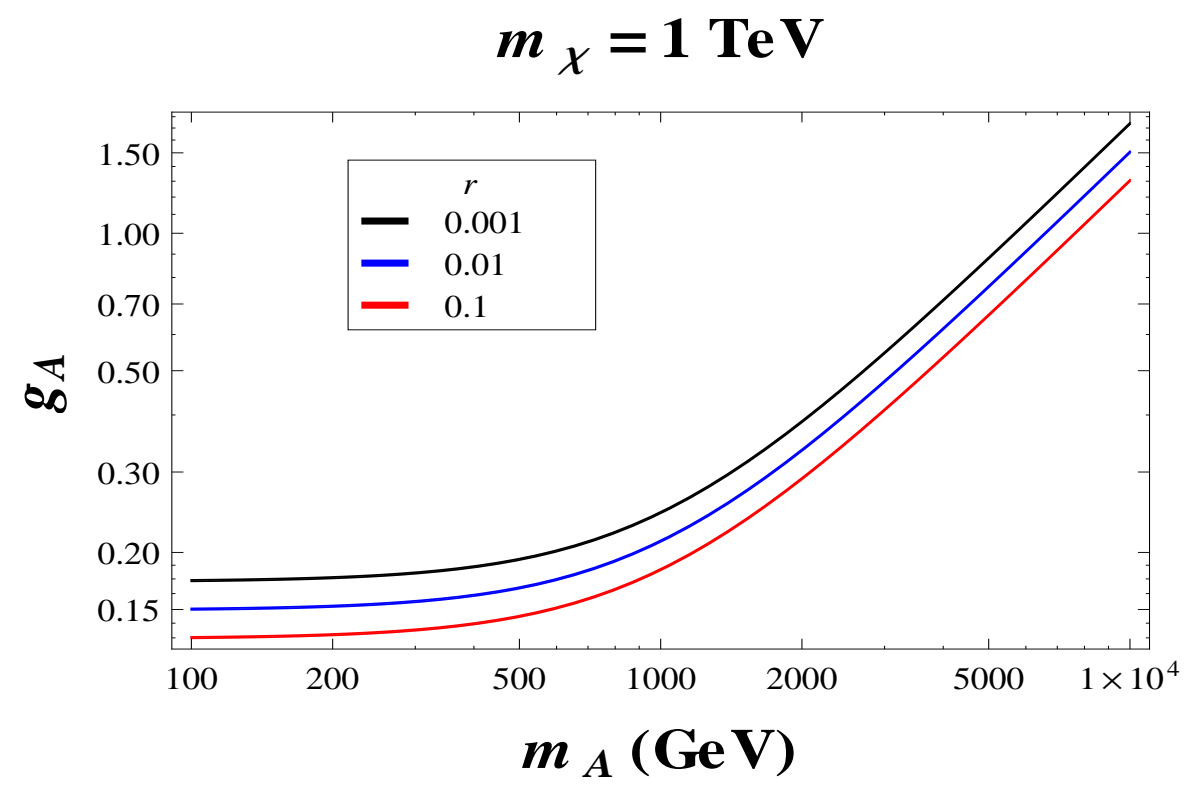

(b) $g_{A}$ vs $m_{A}$ for t/u-channel with $m_{\chi}=1 \mathrm{TeV}$.

Figure 12. In the above figure we have shown the allowed region of $g_{A}$ with respect to the mass of the mediator $m_{A}$, for the $\mathrm{t} / \mathrm{u}$ - channel processes.

where $n_{c}=3$ for quarks and 1 for leptons, $g_{A}$ and $m_{A}$ are the respective coupling and the mass of the mediator. Now taking the following approximation:

$$
s=4 m_{\chi}^{2} /\left(1-v^{2} / 4\right)
$$

we finally get the following simplified expression for the product of annihilation cross- 
section and velocity as:

$$
\sigma v=a+\mathcal{O}\left(v^{2}\right)
$$

where the factor $a$ is given by:

$$
a \approx \frac{n_{c} g^{4} \sqrt{2 m_{\chi}^{2}-m_{f}^{2}}}{8 \pi\left(m^{2}-m_{f}^{2}+m_{\chi}^{2}\right)^{2}}\left[1-\frac{m_{f}^{2}}{m_{\chi}^{2}}\right]^{1 / 2}
$$

The dark matter relic abundance in this case is given as:

$$
\Omega_{D M} h^{2}=\frac{1.07 \times 10^{9}}{J\left(x_{f}\right) g_{*}^{1 / 2} M_{p}}
$$

where $J\left(x_{f}\right)$ for spin-1 mediator $t / u$ channel is given by:

$$
J\left(x_{f}\right)=\int_{x_{f}}^{\infty} \frac{n_{c} g^{4} \sqrt{2 m_{\chi}^{2}-m_{f}^{2}}}{8 \pi\left(m^{2}-m_{f}^{2}+m_{\chi}^{2}\right)^{2} x^{2} f_{\text {membrane }}(x)}\left[1-\frac{m_{f}^{2}}{m_{\chi}^{2}}\right]^{1 / 2} d x
$$

where the function $f_{\text {membrane }}(x)$ is the characteristic parameter for RS single braneworld and this can be expressed in terms of tensor-to-scalar ratio $(r)$ which is given in Eq.(3.29). Now for the GR limiting case of the $f_{\text {membrane }}(x) \rightarrow 1$ and then the relic abundance will only depend on the mass of the Dark Matter $\left(m_{\chi}\right), g_{A}$ the coupling with the spin- 0 mediator and the mass of the mediator $\left(m_{A}\right)$.

$$
\Omega_{D M} h^{2}=\frac{1.07 \times 10^{9} x_{f}}{g_{*}^{1 / 2} M_{p}}\left(\frac{n_{c} g^{4} \sqrt{2 m_{\chi}^{2}-m_{f}^{2}}}{8 \pi\left(m^{2}-m_{f}^{2}+m_{\chi}^{2}\right)^{2}}\left[1-\frac{m_{f}^{2}}{m_{\chi}^{2}}\right]^{1 / 2}\right)^{-1} .
$$

In order to constrain the coupling $\left(g_{A}\right)$ and mass $\left(m_{A}\right)$ we take the present data of the relic abundance $\left(\Omega_{D M} h^{2}=0.1199 \pm 0.0027[56]\right)$ and constrain the function $J\left(x_{f}\right)$, which in turn constrain the coupling $g_{A}$ and $m_{A}$ for a particular tensor-to-scalar ratio $(r)$. We have not shown the GR limiting case as it has been extensively been explored in [55]. In fig. (12(a)) and fig. (12(b)), we have depicted the behaviour of the effective coupling of spin-1 mediator $g_{A}$ with the varying mass the spin- 1 mediator $m_{A}$ for $\mathrm{t} / \mathrm{u}$-channel process with three distinct value of the tensor-to-scalar ratio $r=0.001$, $r=0.01$ and $r=0.1$ respectively in RSII membrane. We also consider three different values of the dark matter mass $m_{\chi}=100 \mathrm{GeV}$ and $m_{\chi}=1 \mathrm{TeV}$ for the $\mathrm{t} / \mathrm{u}$-channel analysis. From fig. (12(a)) and fig. (12(b)), it is clearly observed that the behaviour of the effective coupling of spin- 1 mediator $g_{A}$ with the varying mass the spin-1 mediator $m_{A}$ are similar for all of the three cases, where the coupling decreases with mediator mass. 


\subsection{Majorana dark Matter: spin-1 mediator}

\subsection{1 s-channel analysis}

Further we consider the following Lagrangian for a Majorana dark matter particle, $\chi$, that interacts with the SM via a spin-1 mediator, $V_{\mu}$, within the framework of effective field theory written in RSII membrane as:

$$
\mathcal{L}_{\text {membrane }} \supset\left[\frac{1}{2} g_{\chi_{a}} \bar{\chi} \gamma^{\mu} \gamma^{5} \chi+\bar{f}\left(g_{f_{V}}+g_{f_{a}} \gamma^{5}\right) f\right] V_{\mu}
$$

In fig. (13(a)) and fig. (13(b)), we have explicitly shown the Feynman diagrammatic representation of possible s-channel and t/u-channel processes for dirac dark matter with spin-1 mediator respectively. Now taking equal coupling approximation for the

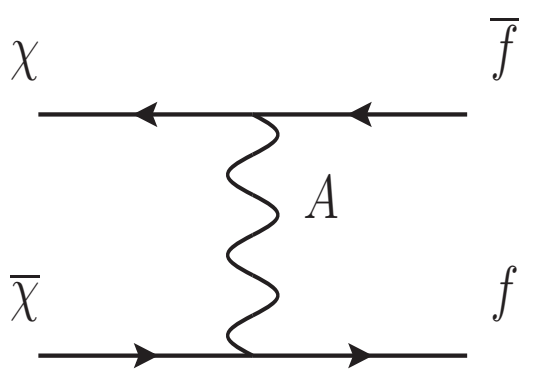

(a) $\chi \bar{\chi} \rightarrow f \bar{f}$ process for s-channel.

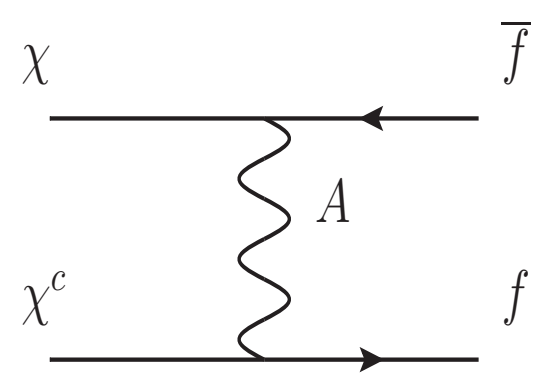

(b) $\chi \chi^{c} \rightarrow f \bar{f}$ process for $\mathrm{t} / \mathrm{u}$-channel.

Figure 13. Feynman diagrammatic representation of s-channel and $t / u$ channel processes for Dirac dark matter with spin-1 mediator.

couplings the cross-section from the above Lagrangian can be computed as:

$$
\sigma=\frac{1}{8 \pi\left(s-4 m_{\chi}^{2}\right)} \int_{t_{-}}^{t_{+}}|\mathcal{M}|^{2} d t
$$

where the matrix element for the $S$ matrix and the symbol $t_{ \pm}$is defined as:

$$
\begin{aligned}
|\mathcal{M}|^{2} & =\frac{2 n_{c} g^{4}}{3 \pi s\left(\left(s-m^{2}\right)^{2}+m^{2} \Gamma^{2}\right)}\left\{4 m_{\chi}^{2} m_{f}^{2}\left(7-6 \frac{s}{m^{2}}+\frac{3 s^{2}}{m^{4}}\right)-4 m_{\chi}^{2} s\right. \\
\left.+s\left(s-4 m_{f}^{2}\right)+\left(s+2 m_{f}^{2}\right)\left(s-4 m_{\chi}^{2}\right)\right\} & \\
t_{ \pm}= & \left(m_{\chi}^{2}+m_{f}^{2}-\frac{s}{2}\right) \pm \frac{\sqrt{\left(s-4 m_{\chi}^{2}\right)\left(s-4 m_{f}^{2}\right)}}{2} .
\end{aligned}
$$

where $n_{c}=3$ for quarks and 1 for leptons, $g_{A}$ and $m_{A}$ are the respective coupling and the mass of the mediator. Now taking the following approximation:

$$
s=4 m_{\chi}^{2} /\left(1+v^{2} / 4\right)
$$




\section{$m_{\chi}=100 \mathrm{GeV}$}

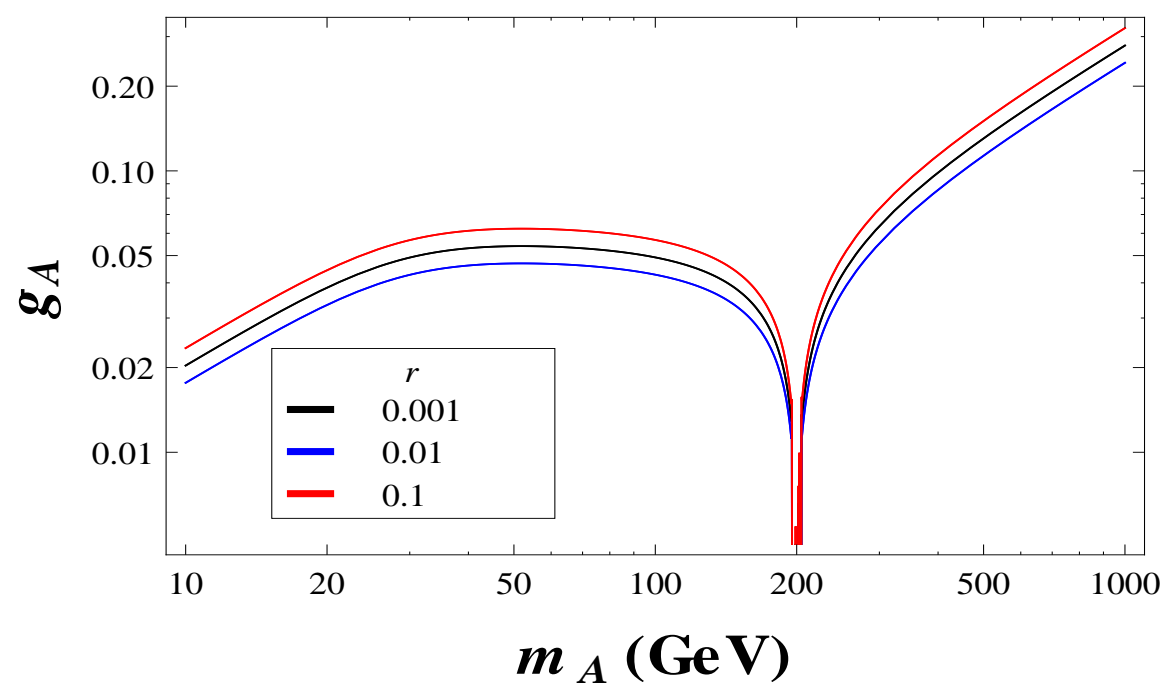

(a) $g_{A}$ vs $m_{A}$ for s-channel with $m_{\chi}=100 \mathrm{GeV}$.

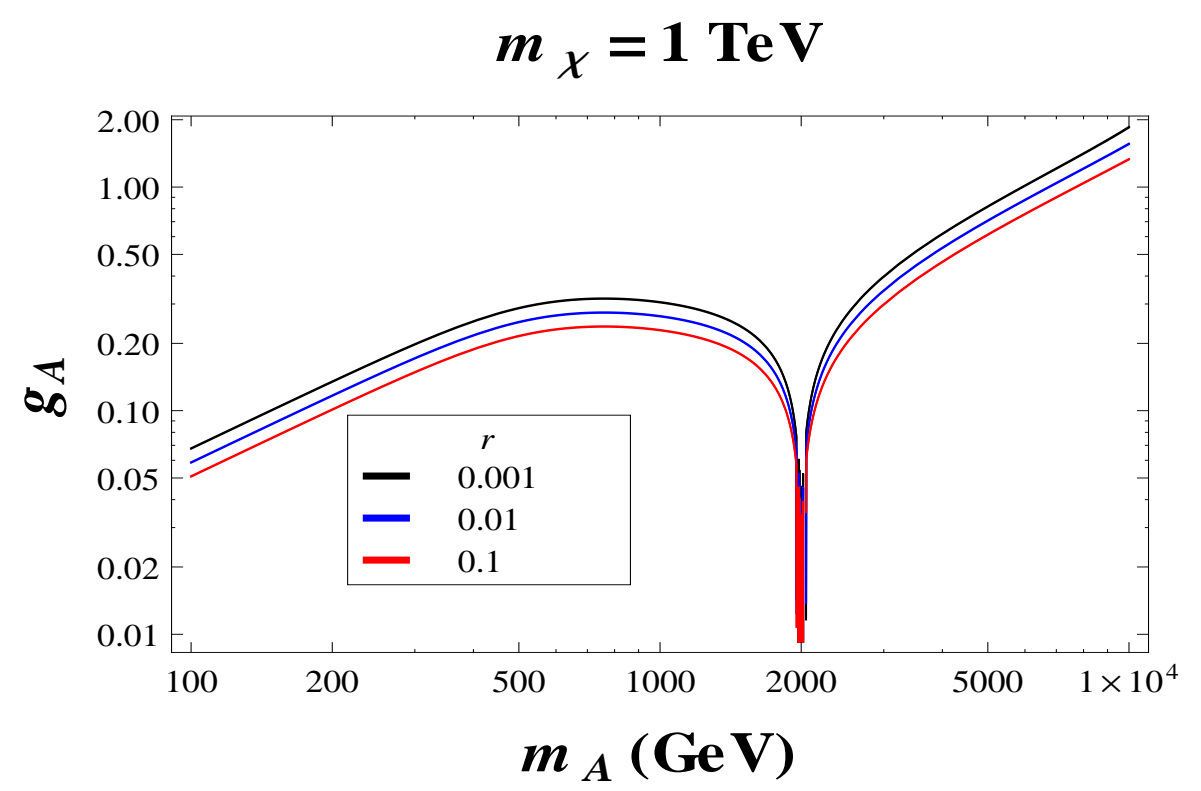

(b) $g_{A}$ vs $m_{A}$ for s-channel with $m_{\chi}=1 \mathrm{TeV}$.

Figure 14. In the above figure we have shown the allowed region of $g_{A}$ with respect to the mass of the mediator $m_{A}$, for the s-channel processes.

we finally get the following simplified expression for the product of annihilation crosssection and velocity as:

$$
\sigma v=a+\mathcal{O}\left(v^{2}\right)
$$


where the factor $a$ is given by:

$$
a=n_{c} g^{4} \frac{m_{f}^{2}}{2 \pi m^{4}} \sqrt{1-\frac{m_{f}^{2}}{m_{\chi}^{2}}} \frac{1-8 \frac{m_{\chi}^{2}}{m^{2}}+16 \frac{m_{\chi}^{4}}{m^{4}}}{1-8 \frac{m_{\chi}^{2}}{m^{2}}+16 \frac{m_{\chi}^{4}}{m^{4}}+\frac{\Gamma^{2}}{m^{2}}},
$$

and the mediator's width to SM fermions is given by:

$$
\Gamma=\sum_{f} \Gamma(A \rightarrow f \bar{f})=\frac{g^{2} n_{c} m}{6 \pi}\left[1-4 \frac{m_{f}^{2}}{m^{2}}\right]^{1 / 2}\left[1-2 \frac{m_{f}^{2}}{m^{2}}\right]
$$

The relic abundance is given as:

$$
\Omega_{D M} h^{2}=\frac{1.07 \times 10^{9}}{J\left(x_{f}\right) g_{*}^{1 / 2} M_{p}}
$$

where $J\left(x_{f}\right)$ for spin-1 mediator s-channel process is given by:

$J\left(x_{f}\right)=\int_{x_{f}}^{\infty} n_{c} g^{4} \frac{m_{f}^{2}}{2 \pi m^{4}} \sqrt{1-\frac{m_{f}^{2}}{m_{\chi}^{2}}} \frac{1-8 \frac{m_{\chi}^{2}}{m^{2}}+16 \frac{m_{\chi}^{4}}{m^{4}}}{\left(1-8 \frac{m_{\chi}^{2}}{m^{2}}+16 \frac{m_{\chi}^{4}}{m^{4}}+\frac{\Gamma^{2}}{m^{2}}\right) x^{2} f_{\text {membrane }}(x)}\left[1-\frac{m_{f}^{2}}{m_{\chi}^{2}}\right]^{1 / 2} d x$.

where the function $f_{\text {membrane }}(x)$ is the characteristic parameter for RS single braneworld and this can be expressed in terms of tensor-to-scalar ratio $(r)$ which is given in Eq.(3.29). Now for the GR limiting case of the $f_{\text {membrane }}(x) \rightarrow 1$ and then the relic abundance will only depend on the mass of the Dark Matter $\left(m_{\chi}\right), g_{A}$ the coupling with the spin- 0 mediator and the mass of the mediator $\left(m_{A}\right)$.

$$
\Omega_{D M} h^{2}=\frac{1.07 \times 10^{9} x_{f}}{g_{*}^{1 / 2} M_{p}}\left(n_{c} g^{4} \frac{m_{f}^{2}}{2 \pi m^{4}} \sqrt{1-\frac{m_{f}^{2}}{m_{\chi}^{2}}} \frac{1-8 \frac{m_{\chi}^{2}}{m^{2}}+16 \frac{m_{\chi}^{4}}{m^{4}}}{1-8 \frac{m_{\chi}^{2}}{m^{2}}+16 \frac{m_{\chi}^{4}}{m^{4}}+\frac{\Gamma^{2}}{m^{2}}}\right)^{-1} .
$$

In order to constrain the coupling $\left(g_{A}\right)$ and mass $\left(m_{A}\right)$ we take the present data of the relic abundance $\left(\Omega_{D M} h^{2}=0.1199 \pm 0.0027[56]\right)$ and constrain the function $J\left(x_{f}\right)$, which in turn constrain the coupling $g_{A}$ and $m_{A}$ for a particular tensor-to-scalar ratio $(r)$. We have not shown the GR limiting case as it has been extensively been explored in [55]. In fig. (14(a)) and fig. (14(b)), we have depicted the behaviour of the effective coupling of spin-1 mediator $g_{A}$ with the varying mass the spin-1 mediator $m_{A}$ for s-channel process with three distinct value of the tensor-to-scalar ratio $r=0.001$, $r=0.01$ and $r=0.1$ respectively in RSII membrane. We also consider two different values of the dark matter mass $m_{\chi}=100 \mathrm{GeV}$ and $m_{\chi}=1 \mathrm{TeV}$ for the s-channel analysis. From fig. (14(a)) and fig. (14(b)), it is clearly observed that the behaviour of the effective coupling of spin- 1 mediator $g_{A}$ with the varying mass the spin-1 mediator $m_{A}$ are similar for both of the cases, where the coupling increases with mediator mass. 


\subsection{2 $\mathrm{t} / \mathrm{u}$ - channel analysis}

Next we consider the following localized interactions for a Majorana dark matter particle, $\chi$, and a spin-1 mediator, $V_{\mu}$, within the framework of effective field theory written in RSII membrane as:

$$
\mathcal{L}_{\text {membrane }} \supset \bar{\chi} \gamma^{\mu}\left(g_{\chi_{V}}+g_{\chi_{a}} \gamma_{5}\right) f V_{\mu}+\bar{f} \gamma^{\mu}\left(g_{f_{V}}+g_{f_{a}}\right) \chi V_{\mu}^{\dagger}
$$

Now taking equal coupling approximation for the couplings the cross-section from the above Lagrangian can be computed as:

$$
\sigma=\frac{1}{8 \pi\left(s-4 m_{\chi}^{2}\right)} \int_{t_{-}}^{t_{+}}|\mathcal{M}|^{2} d t
$$

where the matrix element for the $S$ matrix and the symbol $t_{ \pm}$is defined as:

$$
\begin{aligned}
|\mathcal{M}|^{2} & =2 g_{A}^{2}\left(\frac{s^{2}+u^{2} m_{f}^{4} m_{\chi}^{2}-2 m_{\chi}^{2}(s+u)+m_{f}^{2}\left(6 m_{\chi}^{2}-2(s+u)\right)}{\left(t-m_{A}^{2}\right)^{2}}\right. \\
& \left.+\frac{s^{2}+t^{2} m_{f}^{4} m_{\chi}^{2}-2 m_{\chi}^{2}(s+t)+m_{f}^{2}\left(6 m_{\chi}^{2}-2(s+t)\right)}{\left(u-m_{A}^{2}\right)^{2}}\right) .
\end{aligned}
$$

where $n_{c}=3$ for quarks and 1 for leptons, $g_{A}$ and $m_{A}$ are the respective coupling and the mass of the mediator. Now taking the following approximation:

$$
s=4 m_{\chi}^{2} /\left(1+v^{2} / 4\right)
$$

we finally get the following simplified expression for the product of annihilation crosssection and velocity as:

$$
\sigma v=a+\mathcal{O}\left(v^{2}\right)
$$

where the factor $a$ is given by:

$$
a \approx \frac{n_{c} g_{A}^{4} \sqrt{1-m_{f}^{2} / m_{\chi}^{2}}}{8 \pi m_{A}^{4}\left(m_{A}^{2}-m_{f}^{2}+m_{\chi}^{2}\right)^{2}}\left[8 m_{f}^{6}-8 m_{f}^{4}\left(2 m_{A}^{2}+m_{\chi}^{2}\right)+4 m_{f}^{2}\left(2 m_{A}^{2}+m_{\chi}^{2}\right)^{2}\right] .
$$

The relic abundance is given as:

$$
\Omega_{D M} h^{2}=\frac{1.07 \times 10^{9}}{J\left(x_{f}\right) g_{*}^{1 / 2} M_{p}}
$$

where $J\left(x_{f}\right)$ for spin-1 mediator s-channel process is given by:

$J\left(x_{f}\right)=\int_{x_{f}}^{\infty} \frac{n_{c} g_{A}^{4} \sqrt{1-m_{f}^{2} / m_{\chi}^{2}}}{8 \pi m_{A}^{4}\left(m_{A}^{2}-m_{f}^{2}+m_{\chi}^{2}\right)^{2}} \frac{\left[8 m_{f}^{6}-8 m_{f}^{4}\left(2 m_{A}^{2}+m_{\chi}^{2}\right)+4 m_{f}^{2}\left(2 m_{A}^{2}+m_{\chi}^{2}\right)^{2}\right]}{x^{2} f_{\text {membrane }}(x)} d x$. 


\section{$m_{\chi}=100 \mathrm{GeV}$}

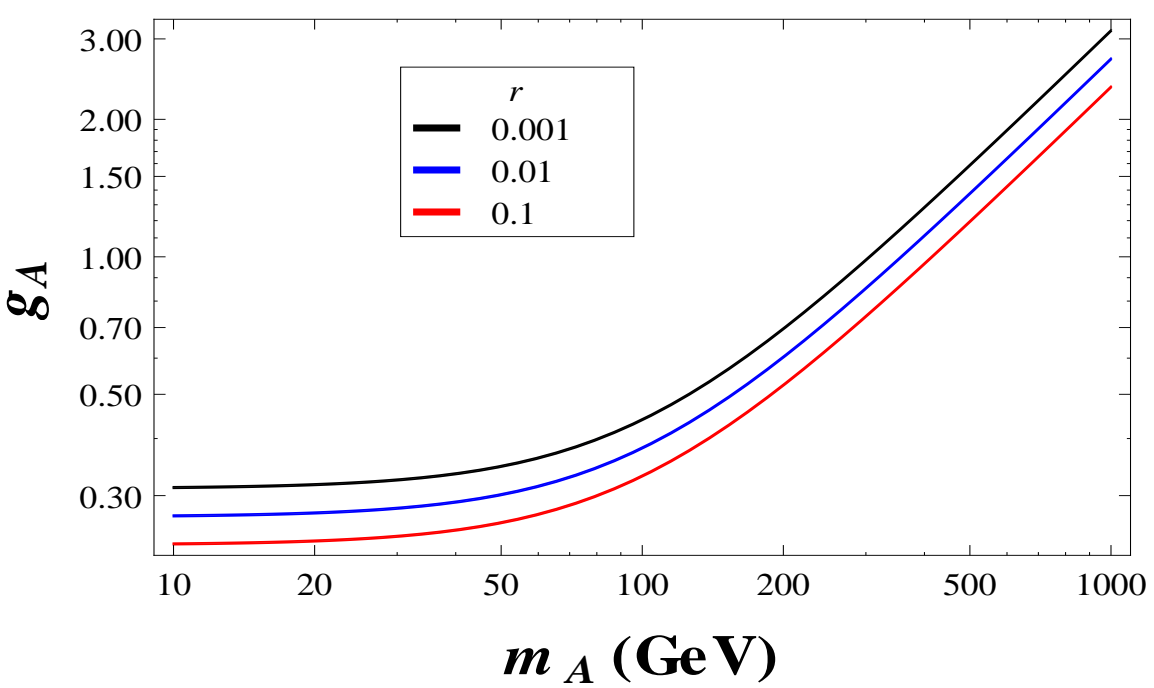

(a) $g_{A}$ vs $m_{A}$ for $\mathrm{t} / \mathrm{u}$-channel with $m_{\chi}=100 \mathrm{GeV}$.

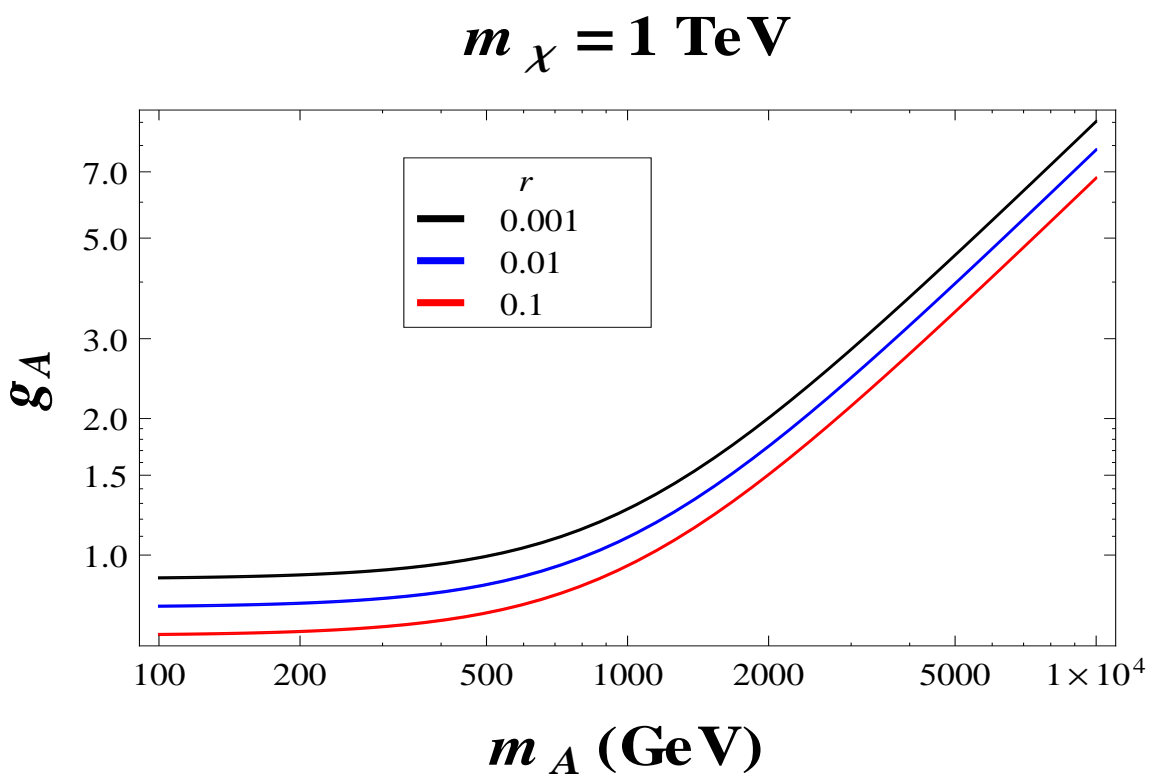

(b) $g_{A}$ vs $m_{A}$ for t/u-channel with $m_{\chi}=1 \mathrm{TeV}$.

Figure 15. In the above figure we have shown the allowed region of $g_{A}$ with respect to the mass of the mediator $m_{A}$, for the $\mathrm{t} / \mathrm{u}$-channel processes.

where the function $f_{\text {membrane }}(x)$ is the characteristic parameter for RS single braneworld and this can be expressed in terms of tensor-to-scalar ratio $(r)$ which is given in Eq.(3.29). Now for the GR limiting case of the $f_{\text {membrane }}(x) \rightarrow 1$ and then the relic abundance will only depend on the mass of the Dark Matter $\left(m_{\chi}\right), g_{A}$ the coupling 
with the spin-0 mediator and the mass of the mediator $\left(m_{A}\right)$.

$$
\begin{aligned}
\Omega_{D M} h^{2} & =\frac{1.07 \times 10^{9} x_{f}}{g_{*}^{1 / 2} M_{p}}\left(\frac{n_{c} g_{A}^{4} \sqrt{1-m_{f}^{2} / m_{\chi}^{2}}}{8 \pi m_{A}^{4}\left(m_{A}^{2}-m_{f}^{2}+m_{\chi}^{2}\right)^{2}}\right. \\
& {\left.\left[8 m_{f}^{6}-8 m_{f}^{4}\left(2 m_{A}^{2}+m_{\chi}^{2}\right)+4 m_{f}^{2}\left(2 m_{A}^{2}+m_{\chi}^{2}\right)^{2}\right]\right)^{-1} . }
\end{aligned}
$$

In order to constrain the coupling $\left(g_{A}\right)$ and mass $\left(m_{A}\right)$ we take the present data of the relic abundance $\left(\Omega_{D M} h^{2}=0.1199 \pm 0.0027[56]\right)$ and constrain the function $J\left(x_{f}\right)$, which in turn constrain the coupling $g_{A}$ and $m_{A}$ for a particular tensor-to-scalar ratio $(r)$. We have not shown the GR limiting case as it has been extensively been explored in [55]. In fig. (15(a)) and fig. (15(b)), we have depicted the behaviour of the effective coupling of spin-0 mediator $g_{A}$ with the varying mass the spin-0 mediator $m_{A}$ for $\mathrm{t} / \mathrm{u}$-channel process with three distinct value of the tensor-to-scalar ratio $r=0.001$, $r=0.01$ and $r=0.1$ respectively in RSII membrane. We also consider three different values of the dark matter mass $m_{\chi}=100 \mathrm{GeV}$ and $m_{\chi}=1 \mathrm{TeV}$ for the $\mathrm{t} / \mathrm{u}$-channel analysis. From fig. (15(a)) and fig. (15(b)), it is clearly observed that the behaviour of the effective coupling of spin-0 mediator $g_{A}$ with the varying mass the spin-0 mediator $m_{A}$ are similar for all of the three cases, where the coupling increases with mediator mass.

\subsection{Complex scalar dark matter: spin-0 mediator}

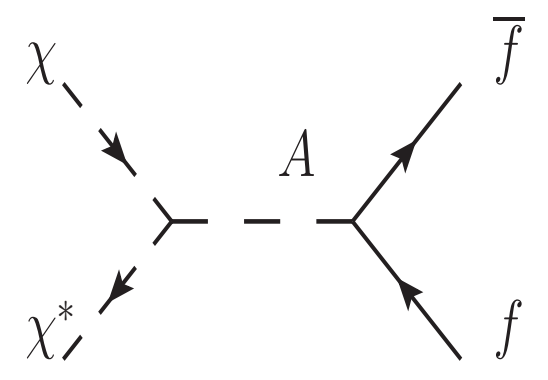

(a) $\chi \chi^{*} \rightarrow f \bar{f}$ process for s-channel.

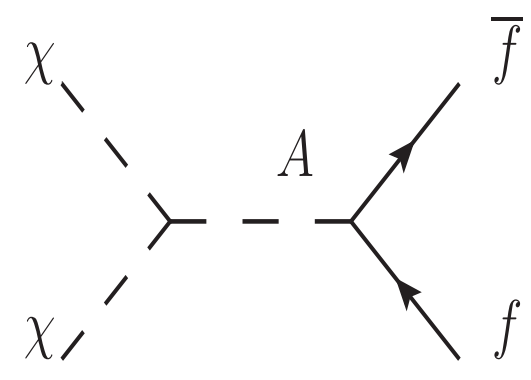

(b) $\chi \chi \rightarrow f \bar{f}$ process for s-channel.

Figure 16. Feynman diagrammatic representation of s-channel processes for complex and real scalar dark matter with spin-0 mediator.

Here we consider the following Lagrangian for for a complex scalar dark matter particle, $\phi$, and a spin-0 mediator, $A$, within the framework of effective field theory written in RSII membrane as:

$$
\mathcal{L}_{\text {membrane }} \supset\left[\mu_{\phi}|\phi|^{2}+\bar{f}\left(\lambda_{f_{s}}+\lambda_{f_{p}} i \gamma^{5}\right) f\right] A .
$$




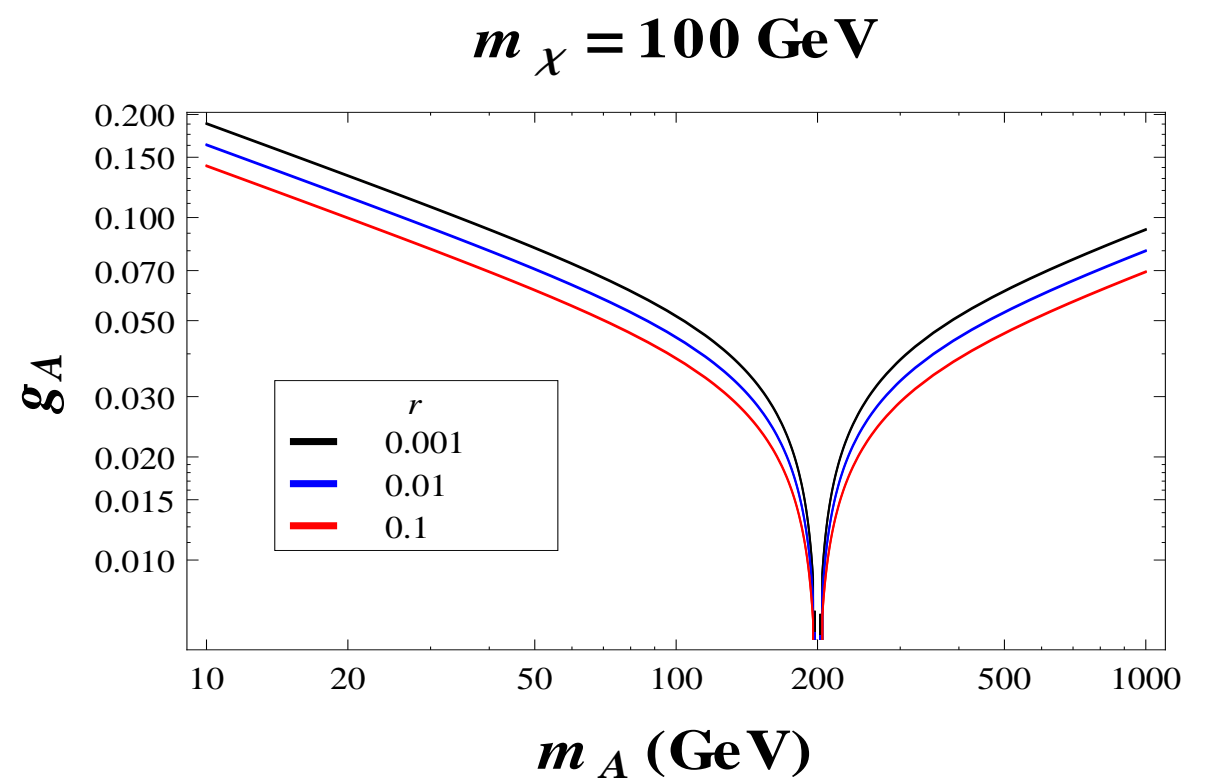

(a) $g_{A}$ vs $m_{A}$ for s-channel with $m_{\chi}=100 \mathrm{GeV}$.

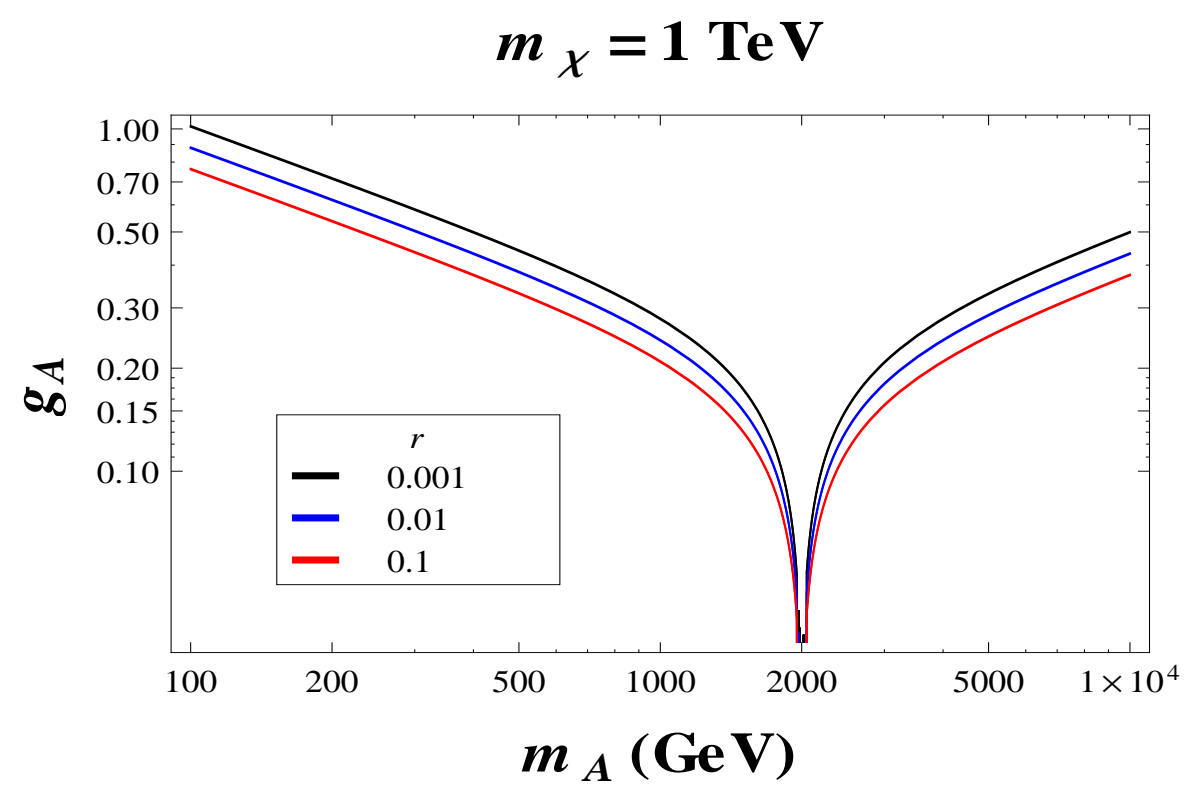

(b) $g_{A}$ vs $m_{A}$ for s-channel with $m_{\chi}=1 \mathrm{TeV}$.

Figure 17. In the above figure we have shown the allowed region of $g_{A}$ with respect to the mass of the mediator $m_{A}$, the upper panel is for the s-channel processes.

In fig. (16(a)), we have explicitly shown the Feynman diagrammatic representation of possible s-channel process for complex scalar dark matter with spin-0 mediator. Now, taking all the couplings to be the same the cross-section becomes:

$$
\sigma=\frac{1}{8 \pi\left(s-4 m_{\chi}^{2}\right)} \int_{t_{-}}^{t_{+}}|\mathcal{M}|^{2} d t
$$


where the matrix element for the S-matrix and the symbol $t_{ \pm}$is given by:

$$
\begin{aligned}
|\mathcal{M}|^{2} & =\frac{n_{c} g^{4}}{\pi s\left(\left(s-m^{2}\right)^{2}+m^{2} \Gamma^{2}\right)}\left\{s-2 m_{f}^{2}\right\} \\
t_{ \pm} & =\left(m_{\chi}^{2}+m_{f}^{2}-\frac{s}{2}\right) \pm \frac{\sqrt{\left(s-4 m_{\chi}^{2}\right)\left(s-4 m_{f}^{2}\right)}}{2} .
\end{aligned}
$$

where $n_{c}=3$ for quarks and 1 for leptons, $g_{A}$ and $m_{A}$ are the respective coupling and the mass of the mediator. Now taking the following approximation:

$$
s=4 m_{\chi}^{2} /\left(1+v^{2} / 4\right)
$$

we finally get the following simplified expression for the product of annihilation crosssection and velocity as:

$$
\sigma v=a+\mathcal{O}\left(v^{2}\right)
$$

where the factor $a$ is given by:

$$
a=n_{c} g^{4} \frac{m^{2}}{2 \pi} \sqrt{1-\frac{m_{f}^{2}}{m_{\chi}^{2}}} \frac{1-\frac{m^{2} f}{2 m_{\chi}^{2}}}{\left(m^{2}-4 m_{\chi}^{2}\right)^{2}+m^{2} \Gamma^{2}}
$$

and the mediator's width to SM fermions is given by:

$$
\Gamma=\frac{g^{4} n_{c} m}{4 \pi}\left[1-4 \frac{m_{f}^{2}}{m^{2}}\right]^{1 / 2}\left[1-2 \frac{m_{f}^{2}}{m^{2}}\right] .
$$

The dark matter relic abundance in this case is given as:

$$
\Omega_{D M} h^{2}=\frac{1.07 \times 10^{9}}{J\left(x_{f}\right) g_{*}^{1 / 2} M_{p}}
$$

where $J\left(x_{f}\right)$ for complex dark matter with spin-0 mediator is given by:

$$
J\left(x_{f}\right)=\int_{x_{f}}^{\infty} n_{c} g^{4} \frac{m^{2}}{2 \pi} \sqrt{1-\frac{m_{f}^{2}}{m_{\chi}^{2}}} \frac{1-\frac{m^{2} f}{2 m_{\chi}^{2}}}{\left(\left(m^{2}-4 m_{\chi}^{2}\right)^{2}+m^{2} \Gamma^{2}\right) x^{2} f_{\text {membrane }}(x)} d x .
$$

where the function $f_{\text {membrane }}(x)$ is the characteristic parameter for $\mathrm{RS}$ single braneworld and this can be expressed in terms of tensor-to-scalar ratio $(r)$ which is given in Eq.(3.29). Now for the GR limiting case of the $f_{\text {membrane }}(x) \rightarrow 1$ and then the relic abundance will only depend on the mass of the Dark Matter $\left(m_{\chi}\right), g_{A}$ the coupling with the spin-0 mediator and the mass of the mediator $\left(m_{A}\right)$.

$$
\Omega_{D M} h^{2}=\frac{1.07 \times 10^{9} x_{f}}{g_{*}^{1 / 2} M_{p}}\left(n_{c} g^{4} \frac{m^{2}}{2 \pi} \sqrt{1-\frac{m_{f}^{2}}{m_{\chi}^{2}}} \frac{1-\frac{m^{2} f}{2 m_{\chi}^{2}}}{\left(m^{2}-4 m_{\chi}^{2}\right)^{2}+m^{2} \Gamma^{2}}\right)^{-1} .
$$


In order to constrain the coupling $\left(g_{A}\right)$ and mass $\left(m_{A}\right)$ we take the present data of the relic abundance $\left(\Omega_{D M} h^{2}=0.1199 \pm 0.0027[56]\right)$ and constrain the function $J\left(x_{F}\right)$, which in turn constrain the coupling $g_{A}$ and $m_{A}$ for a particular tensor-to-scalar ratio $(r)$. We have not shown the GR limiting case as it has been extensively been explored in [55]. In fig. (17(a)) and fig. (17(b)), we have depicted the behaviour of the effective coupling of spin-0 mediator $g_{A}$ with the varying mass the spin- 0 mediator $m_{A}$ for s-channel process with three distinct value of the tensor-to-scalar ratio $r=0.001$, $r=0.01$ and $r=0.1$ respectively in RSII membrane. We also consider two different values of the dark matter mass $m_{\chi}=100 \mathrm{GeV}$ and $m_{\chi}=1 \mathrm{TeV}$ for the s-channel analysis. From fig. (17(a)) and fig. (17(b)), it is clearly observed that the behaviour of the effective coupling of spin-0 mediator $g_{A}$ with the varying mass the spin-0 mediator $m_{A}$ are similar for all of the three cases and also sensitive in the vicinity of $m_{A}=2 \times 10^{2} \mathrm{GeV}$ and $m_{A}=2 \times 10^{3} \mathrm{GeV}$ respectively as it has a resonance (i.e $2 m_{X}=m_{A}$ ). Most importantly, in both the sides of $m_{A}=2 \times 10^{2} \mathrm{GeV}$ and $m_{A}=2 \times 10^{3} \mathrm{GeV}$ the coupling of spin- 0 mediator $g_{A}$ behave in completely opposite manner.

Now, for real scalar dark matter with spin-0 mediator we can also write down the the Lagrangian for a real scalar DM particle, $\phi$, that interacts with the SM via a spin-0 meidator, A, within the framework of effective field theory written in RSII membrane as:

$$
\mathcal{L}_{\text {membrane }} \supset\left[\frac{1}{2} \mu_{\phi} \phi^{2}+\bar{f}\left(\lambda_{f_{S}}+\lambda_{f_{p}} i \gamma^{5}\right) f\right] A .
$$

In fig. (16(b)), we have explicitly shown the Feynman diagrammatic representation of possible s-channel process for real scalar dark matter with spin-0 mediator. Hence after taking all the coupling same and further taking the following approximation:

$$
s=4 m_{\chi}^{2} /\left(1+v^{2} / 4\right)
$$

we finally get the following simplified expression for the product of annihilation crosssection and velocity as:

$$
\sigma v=a+\mathcal{O}\left(v^{2}\right)
$$

where the factor $a$ is given by:

$$
a=\frac{n_{c} g_{A}^{4}}{4 \pi\left(m_{A}^{2}-4 m_{\phi}^{2}\right)^{2}} \sqrt{1-m_{f}^{2} / m_{\chi}^{2}}\left[2-m_{f}^{2} / m_{\phi}^{2}\right] .
$$

\subsection{Complex scalar dark matter: spin-1 mediator}

Here we consider the following interactions for a complex scalar dark matter particle, $\phi$, and a spin-0 mediator, $A$, within the framework of effective field theory written in RSII membrane as:

$$
\mathcal{L}_{\text {membrane }} \supset\left[i g_{\phi} \phi^{\dagger} \overleftrightarrow{\partial_{\mu}} \phi+\bar{f} \gamma_{\mu}\left(g_{f v}+g_{f a} \gamma^{5}\right) f\right] V^{\mu}
$$




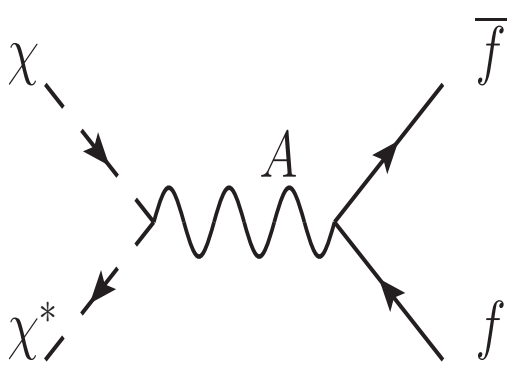

(a) $\chi \chi^{*} \rightarrow f \bar{f}$ process for s-channel.

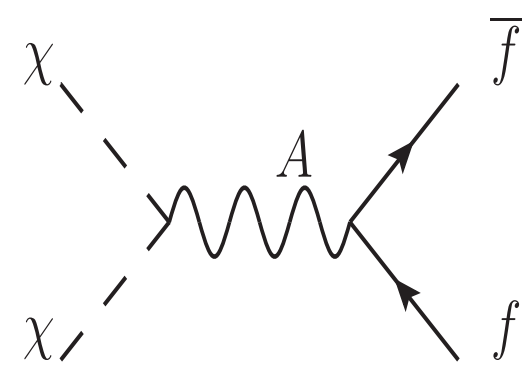

(b) $\chi \chi \rightarrow f \bar{f}$ process for s-channel.

Figure 18. Feynman diagrammatic representation of s-channel processes for complex and real scalar dark matter with spin-1 mediator.

$$
m_{\chi}=100 \mathrm{GeV}
$$

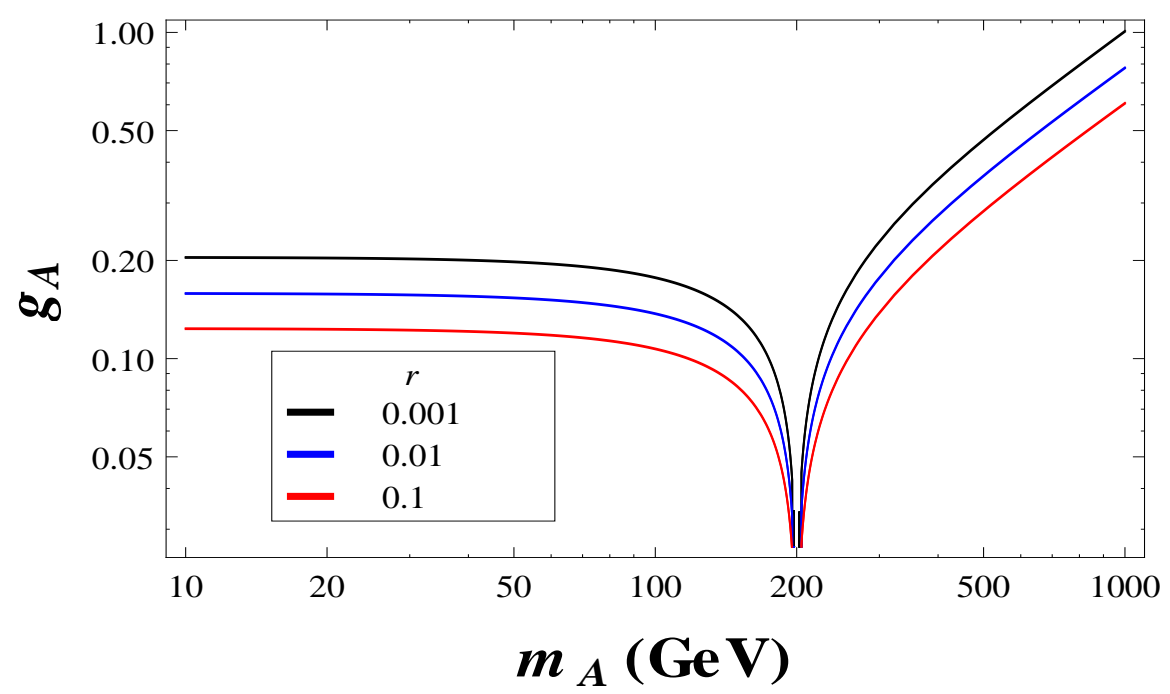

(a) $g_{A}$ vs $m_{A}$ for s-channel with $m_{\chi}=100 \mathrm{GeV}$.

Figure 19. In the above figure we have shown the allowed region of $g_{A}$ with respect to the mass of the mediator $m_{A}$, the upper panel is for the s-channel processes.

In fig. (18(a)) and fig. (18(b)), we have explicitly shown the Feynman diagrammatic representation of possible s-channel for complex and real scalar dark matter with spin-1 mediator. The cross-section from the above Lagrangian after taking all the coupling equal is given as:

$$
\sigma=\frac{1}{8 \pi\left(s-4 m_{\chi}^{2}\right)} \int_{t_{-}}^{t_{+}}|\mathcal{M}|^{2} d t
$$


where the matrix element for the S-matrix and the symbol $t_{ \pm}$is defined as:

$$
\begin{aligned}
|\mathcal{M}|^{2} & =\frac{8 n_{c} g^{4}}{3 \pi\left(\left(s-m^{2}\right)^{2}+m^{2} \Gamma^{2}\right)}\left(1-\frac{2 m_{f}^{2}}{s}\right) \\
t_{ \pm} & =\left(m_{\chi}^{2}+m_{f}^{2}-\frac{s}{2}\right) \pm \frac{\sqrt{\left(s-4 m_{\chi}^{2}\right)\left(s-4 m_{f}^{2}\right)}}{2} .
\end{aligned}
$$

where $n_{c}=3$ for quarks and 1 for leptons, $g_{A}$ and $m_{A}$ are the respective coupling and the mass of the mediator. Further taking the following approximation:

$$
s=4 m_{\chi}^{2} /\left(1+v^{2} / 4\right)
$$

we finally get the following simplified expression for the product of annihilation crosssection and velocity as:

$$
\sigma v=b v^{2}+\mathcal{O}\left(v^{3}\right)
$$

where the factor $b$ is given by:

$$
b=n_{c} g^{4} \frac{m_{\chi}^{2}}{3 \pi} \sqrt{1-\frac{m_{f}^{2}}{m_{\chi}^{2}}} \frac{1-\frac{m^{2} f}{2 m_{\chi}^{2}}}{\left(m^{2}-4 m_{\chi}^{2}\right)^{2}+m^{2} \Gamma^{2}},
$$

and the mediator's width to SM fermions is given by:

$$
\Gamma=\frac{g^{2} n_{c} m}{6 \pi} \sqrt{1-\frac{4 m_{f}^{2}}{m^{2}}}\left[1-\frac{m_{f}^{2}}{m^{2}}\right]
$$

The dark matter relic abundance in this case is given as:

$$
\Omega_{D M} h^{2}=\frac{1.07 \times 10^{9}}{J\left(x_{f}\right) g_{*}^{1 / 2} M_{p}}
$$

where $J\left(x_{f}\right)$ for complex scalar dark matter with spin-1 mediator is given by:

$$
J\left(x_{f}\right)=\int_{x_{f}}^{\infty} n_{c} g^{4} \frac{m_{\chi}^{2}}{3 \pi} \sqrt{1-\frac{m_{f}^{2}}{m_{\chi}^{2}}} \frac{1-\frac{m^{2} f}{2 m_{\chi}^{2}}}{\left(\left(m^{2}-4 m_{\chi}^{2}\right)^{2}+m^{2} \Gamma^{2}\right) x^{3} f_{\text {membrane }}(x)} d x
$$

where the function $f_{\text {membrane }}(x)$ is the characteristic parameter for RS single braneworld and this can be expressed in terms of tensor-to-scalar ratio $(r)$ which is given in Eq.(3.29). Now for the GR limiting case of the $f_{\text {membrane }}(x) \rightarrow 1$ and then the relic abundance will only depend on the mass of the Dark Matter $\left(m_{\chi}\right), g_{A}$ the coupling with the spin- 0 mediator and the mass of the mediator $\left(m_{A}\right)$.

$$
\Omega_{D M} h^{2}=\frac{1.07 \times 10^{9} x_{f}^{2}}{g_{*}^{1 / 2} M_{p}}\left(n_{c} g^{4} \frac{m_{\chi}^{2}}{3 \pi} \sqrt{1-\frac{m_{f}^{2}}{m_{\chi}^{2}}} \frac{1-\frac{m^{2} f}{2 m_{\chi}^{2}}}{\left(m^{2}-4 m_{\chi}^{2}\right)^{2}+m^{2} \Gamma^{2}}\right)^{-1} .
$$


In order to constrain the coupling $\left(g_{A}\right)$ and mass $\left(m_{A}\right)$ we take the present data of the relic abundance $\left(\Omega_{D M} h^{2}=0.1199 \pm 0.0027[56]\right)$ and constrain the function $J\left(x_{f}\right)$, which in turn constrain the coupling $g_{A}$ and $m_{A}$ for a particular tensor-toscalar ratio $(r)$. We have not shown the GR limiting case as it has been extensively been explored in [55]. In fig. (19(a)), we have depicted the behaviour of the effective coupling of spin-0 mediator $g_{A}$ with the varying mass the spin- 0 mediator $m_{A}$ for s-channel process with three distinct value of the tensor-to-scalar ratio $r=0.001$, $r=0.01$ and $r=0.1$ respectively in RSII membrane. We have only considered one value of the dark matter mass which is $m_{\chi}=100 \mathrm{GeV}$, cause as go to higher values like $\mathcal{O}(\mathrm{TeV})$ the required coupling is going more than $\mathcal{O}(1)$, for the s-channel analysis. From fig. (19(a)), it is clearly observed that the behaviour of the effective coupling of spin-0 mediator $g_{A}$ with the varying mass the spin-0 mediator $m_{A}$ is sensitive in the vicinity of $m_{A}=2 \times 10^{2} \mathrm{GeV}$ as it is reaching a resonance (i.e $2 m_{X}=m_{A}$ ). Most importantly, in both the sides of $m_{A}=2 \times 10^{2} \mathrm{GeV}$ the coupling of spin-0 mediator $g_{A}$ behave in completely opposite manner. Now for the real vector dark matter with spin-1 mediator, the real scalar s-channel interaction vanishes identically, so that real scalar dark matter cannot couple to a vector at tree level of the effective field theory.

\subsection{Complex vector dark matter: spin-0 mediator}

Consider the following interaction for a complex vector dark matter, $\chi_{\mu}$, and a spin-0 mediator, A:

$$
\mathcal{L}_{\text {membrane }} \supset\left[\mu_{\chi} \chi^{\mu} \chi_{\mu}^{\dagger}+\bar{f}\left(\lambda_{f_{S}}+\lambda_{f_{p}} i \gamma^{5}\right) f\right] A
$$

In fig. (20(a)), we have explicitly shown the Feynman diagramatic representation of

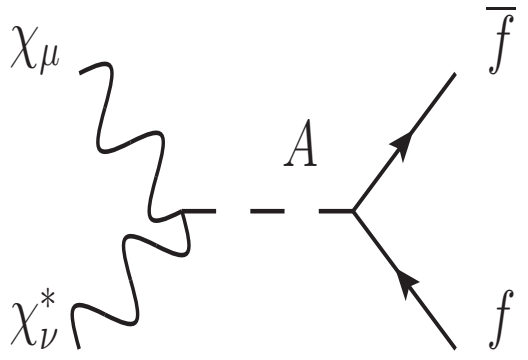

(a) $\chi_{\mu} \chi_{\nu}^{*} \rightarrow f \bar{f}$ process for s-channel.

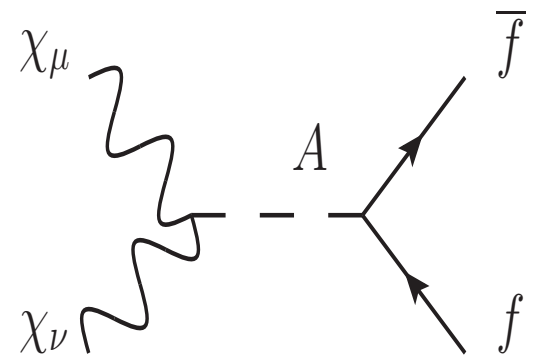

(b) $\chi_{\mu} \chi_{\nu} \rightarrow f \bar{f}$ process for s-channel.

Figure 20. Feynman diagrammatic representation of s-channel processes for complex and real vector dark matter with spin-0 mediator.

possible s-channel for complex vector dark matter with spin-0 mediator. 
$m_{\chi}=100 \mathrm{GeV}$

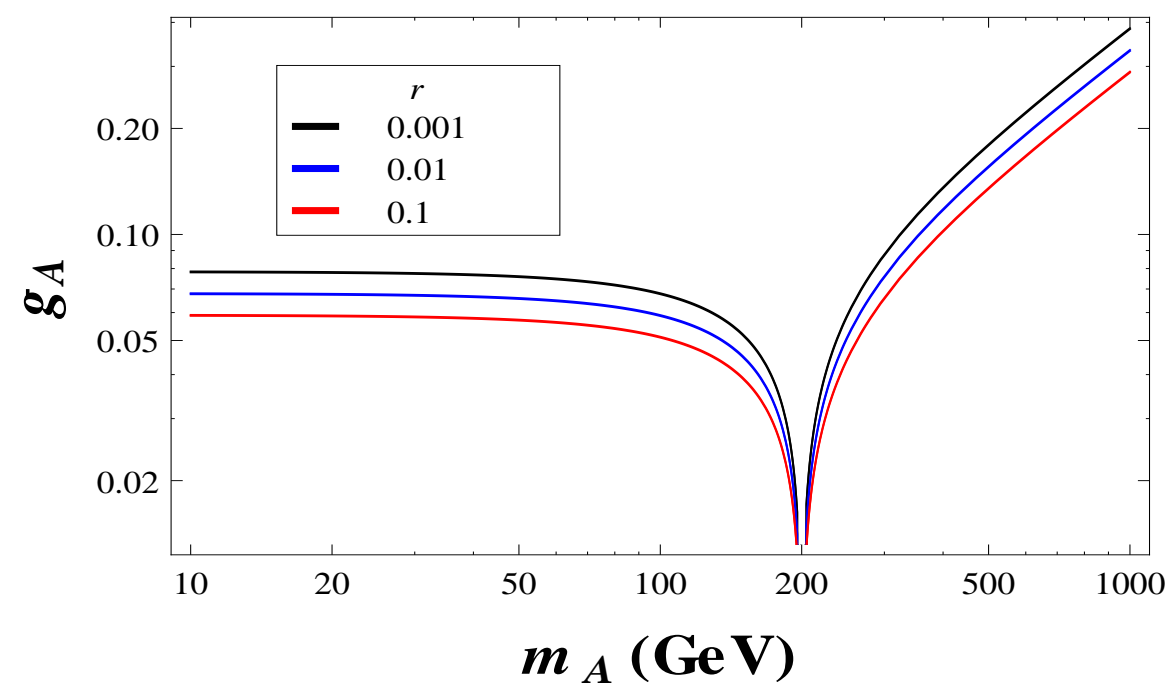

(a) $g_{A}$ vs $m_{A}$ for s-channel with $m_{\chi}=100 \mathrm{GeV}$.

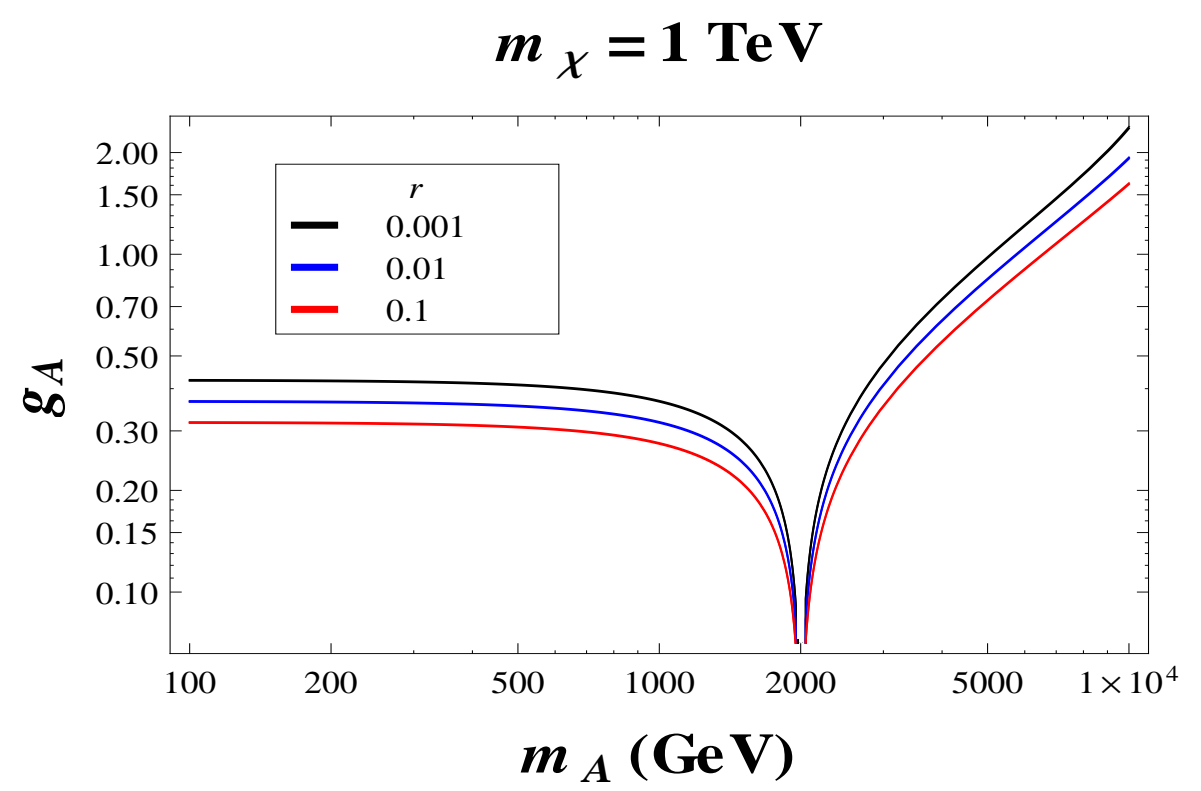

(b) $g_{A}$ vs $m_{A}$ for s-channel with $m_{\chi}=1 \mathrm{TeV}$.

Figure 21. In the above figure we have shown the allowed region of $g_{A}$ with respect to the mass of the mediator $m_{A}$, the upper panel is for the s-channel processes.

The cross-section from the above Lagrangian after taking all the coupling equal is given as:

$$
\sigma=\frac{1}{8 \pi\left(s-4 m_{\chi}^{2}\right)} \int_{t_{-}}^{t_{+}}|\mathcal{M}|^{2} d t
$$


where the matrix element for the S-matrix and the symbol $t_{ \pm}$is defined as:

$$
\begin{aligned}
|\mathcal{M}|^{2} & =\frac{n_{c} g^{4}}{9 \pi\left(\left(s-m^{2}\right)^{2}+m^{2} \Gamma^{2}\right)}\left[\frac{s}{m_{\chi}^{2}}\left[\frac{s}{4 m_{\chi}^{2}}-1\right]+3\right] \\
t_{ \pm} & =\left(m_{\chi}^{2}+m_{f}^{2}-\frac{s}{2}\right) \pm \frac{\sqrt{\left(s-4 m_{\chi}^{2}\right)\left(s-4 m_{f}^{2}\right)}}{2}
\end{aligned}
$$

where $n_{c}=3$ for quarks and 1 for leptons, $g_{A}$ and $m_{A}$ are the respective coupling and the mass of the mediator. Further taking the following approximation:

$$
s=4 m_{\chi}^{2} /\left(1+v^{2} / 4\right)
$$

we finally get the following simplified expression for the product of annihilation crosssection and velocity as:

$$
\sigma v=a+\mathcal{O}\left(v^{2}\right)
$$

where the factor $a$ is given by:

$$
a=n_{c} g^{4} \frac{m_{\chi}^{2}}{6 \pi} \sqrt{1-\frac{m_{f}^{2}}{m_{\chi}^{2}}} \frac{1-\frac{m^{2} f}{2 m_{\chi}^{2}}}{\left(m^{2}-4 m_{\chi}^{2}\right)^{2}+m^{2} \Gamma^{2}}
$$

and the mediator's width to SM fermions is given by:

$$
\Gamma=\frac{g^{2} n_{c} m}{6 \pi} \sqrt{1-\frac{4 m_{f}^{2}}{m^{2}}}\left[1-\frac{m_{f}^{2}}{m^{2}}\right] .
$$

The dark matter relic abundance in this case is given by:

$$
\Omega_{D M} h^{2}=\frac{1.07 \times 10^{9}}{J\left(x_{f}\right) g_{*}^{1 / 2} M_{p}}
$$

where $J\left(x_{f}\right)$ for complex vector dark matter with spin-0 mediator is given by:

$$
J\left(x_{f}\right)=\int_{x_{f}}^{\infty} n_{c} g^{4} \frac{m_{\chi}^{2}}{6 \pi} \sqrt{1-\frac{m_{f}^{2}}{m_{\chi}^{2}}} \frac{1-\frac{m^{2} f}{2 m_{\chi}^{2}}}{\left(\left(m^{2}-4 m_{\chi}^{2}\right)^{2}+m^{2} \Gamma^{2}\right) x^{2} f_{\text {membrane }}(x)} d x .
$$

where the function $f_{\text {membrane }}(x)$ is the characteristic parameter for RS single braneworld and this can be expressed in terms of tensor-to-scalar ratio $(r)$ which is given in Eq.(3.29). Now for the GR limiting case of the $f_{\text {membrane }}(x) \rightarrow 1$ and then the relic abundance will only depend on the mass of the Dark Matter $\left(m_{\chi}\right), g_{A}$ the coupling with the spin-0 mediator and the mass of the mediator $\left(m_{A}\right)$.

$$
\Omega_{D M} h^{2}=\frac{1.07 \times 10^{9} x_{f}}{g_{*}^{1 / 2} M_{p}}\left(n_{c} g^{4} \frac{m_{\chi}^{2}}{6 \pi} \sqrt{1-\frac{m_{f}^{2}}{m_{\chi}^{2}}} \frac{1-\frac{m^{2} f}{2 m_{\chi}^{2}}}{\left(m^{2}-4 m_{\chi}^{2}\right)^{2}+m^{2} \Gamma^{2}}\right)^{-1} .
$$


In order to constrain the coupling $\left(g_{A}\right)$ and mass $\left(m_{A}\right)$ we take the present data of the relic abundance $\left(\Omega_{D M} h^{2}=0.1199 \pm 0.0027[56]\right)$ and constrain the function $J(x)$, which in turn constrain the coupling $g_{A}$ and $m_{A}$ for a particular tensor-to-scalar ratio $(r)$. We have not shown the GR limiting case as it has been extensively been explored in [55]. In fig. (21(a)) and fig. (21(b)), we have depicted the behaviour of the effective coupling of spin-0 mediator $g_{A}$ with the varying mass the spin- 0 mediator $m_{A}$ for s-channel process with three distinct value of the tensor-to-scalar ratio $r=0.001$, $r=0.01$ and $r=0.1$ respectively in RSII membrane. We also consider two different values of the dark matter mass $m_{\chi}=100 \mathrm{GeV}$ and $m_{\chi}=1 \mathrm{TeV}$ for the s-channel analysis. From fig. (21(a)) and fig. (21(b)), it is clearly observed that the behaviour of the effective coupling of spin-0 mediator $g_{A}$ with the varying mass the spin-0 mediator $m_{A}$ are almost similar for both of the cases and also sensitive in the vicinity of $m_{A}=2 \times 10^{2} \mathrm{GeV}$ and $m_{A}=2 \times 10^{3} \mathrm{GeV}$ respectively as it has a resonance (i.e $\left.2 m_{X}=m_{A}\right)$. Most importantly, in both the sides of $m_{A}=2 \times 10^{2} \mathrm{GeV}$ and $m_{A}=2 \times 10^{3} \mathrm{GeV}$ the coupling of spin- 0 mediator $g_{A}$ behave in completely opposite manner.

Now, similar to the earlier case of complex vector dark matter that interacts with SM through spin-0 mediator, a real vector matter Lagrangian will look like:

$$
\mathcal{L}_{\text {membrane }} \supset\left[\frac{1}{2} \mu_{\chi} \chi^{\mu} \chi_{\mu}^{\dagger}+\bar{f}\left(\lambda_{f_{S}}+\lambda_{f_{p}} i \gamma^{5}\right) f\right] A
$$

and after taking all the couplings equal the thermally averaged cross-section is same as mentioned in the previous subsection for the complex vector dark matter. In fig. (20(b)), we have explicitly shown the Feynman diagrammatic representation of possible s-channel for real vector dark matter with spin-0 mediator.

\subsection{Complex vector dark matter: spin-1 mediator}

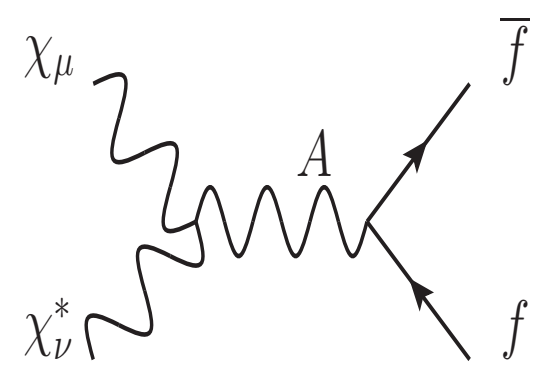

(a) $\chi_{\mu} \chi_{\nu}^{*} \rightarrow f \bar{f}$ process for s-channel.

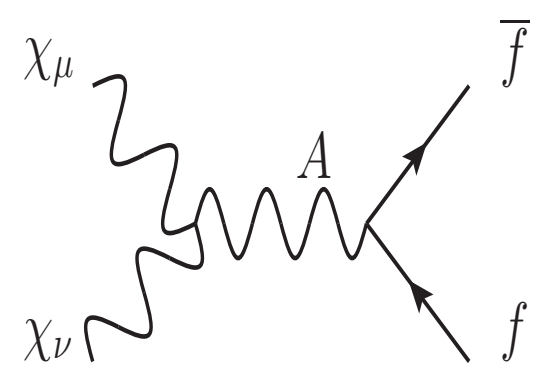

(b) $\chi_{\mu} \chi_{\nu} \rightarrow f \bar{f}$ process for s-channel.

Figure 22. Feynman diagrammatic representation of s-channel processes for complex and real vector dark matter with spin-1 mediator. 


\section{$m_{\chi}=100 \mathrm{GeV}$}

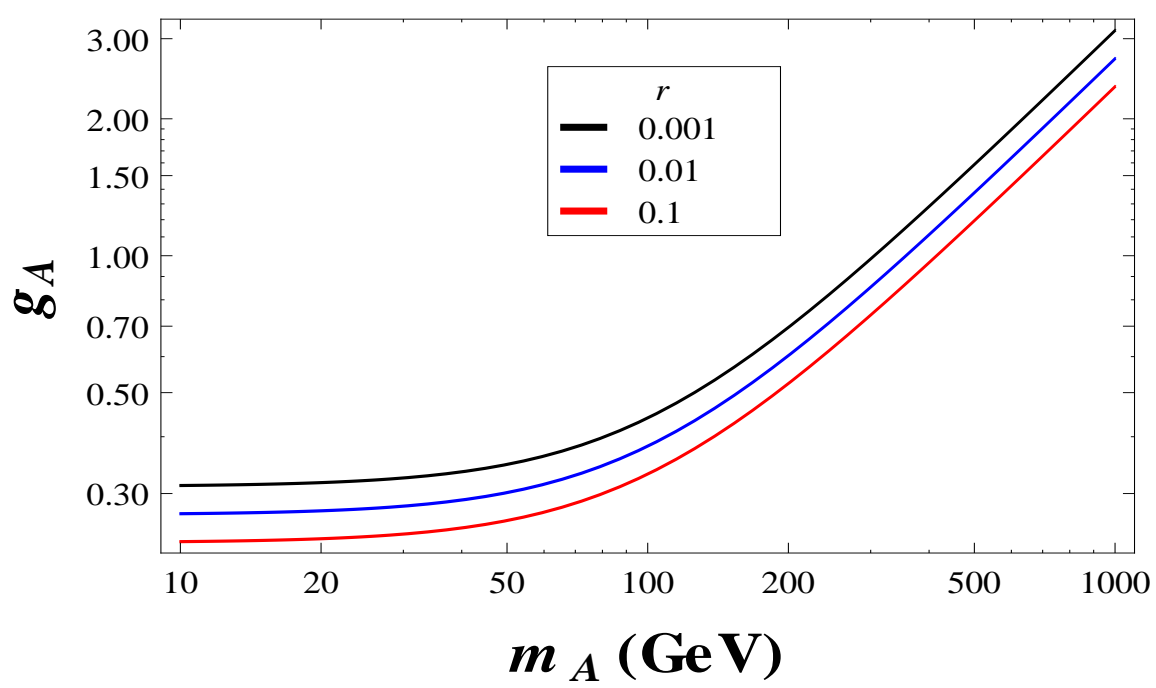

(a) $g_{A}$ vs $m_{A}$ for s-channel with $m_{\chi}=100 \mathrm{GeV}$.

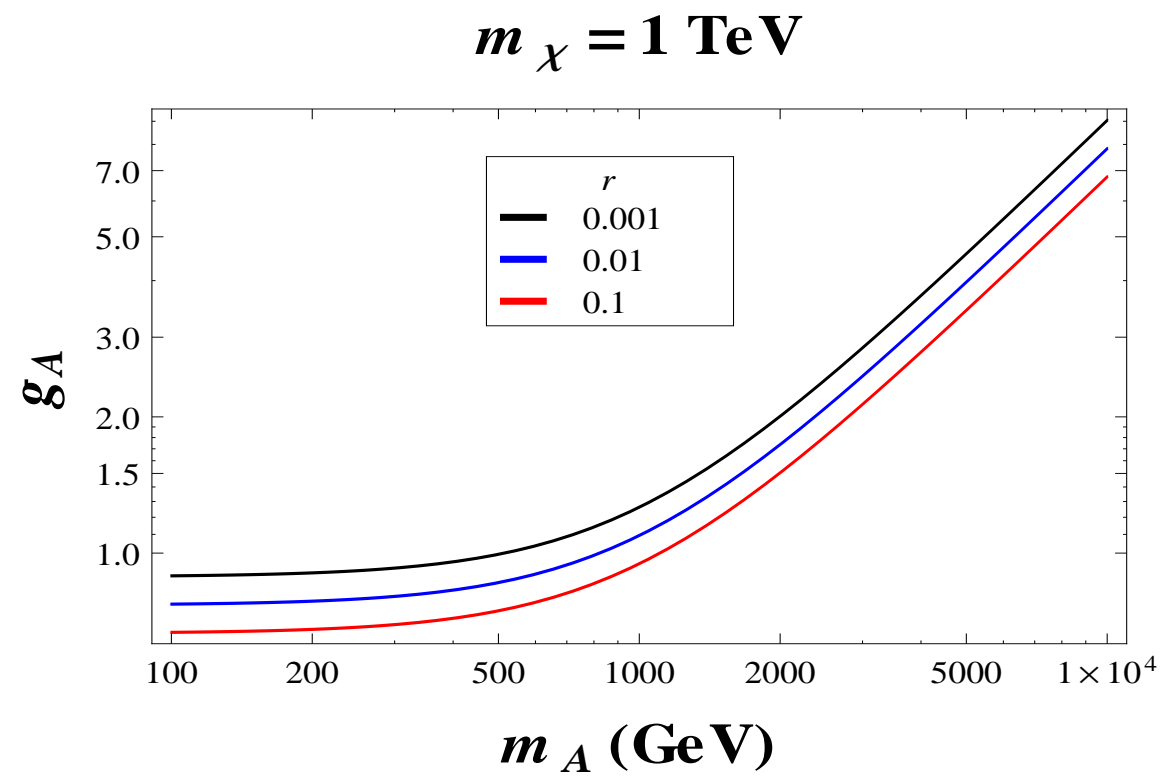

(b) $g_{A}$ vs $m_{A}$ for s-channel with $m_{\chi}=1 \mathrm{TeV}$.

Figure 23. In the above figure we have shown the allowed region of $g_{A}$ with respect to the mass of the mediator $m_{A}$, the above processes are s-channel.

Here we consider the Lagrangian of complex scalar dark matter with spin-1 mediator is given as:

$$
\mathcal{L}_{\text {membrane }} \supset\left[g_{X}\left(X^{\dagger \mu} \partial_{\nu} X^{\nu}+\text { h.c }\right)+\bar{f} \gamma^{\mu}\left(g_{f v}+g_{f a} \gamma^{5}\right) f\right] V_{\mu}
$$

In fig. (22(a)) and fig. (22(b)), we have explicitly shown the Feynman diagrammatic 
representation of possible s-channel for complex and real vector dark matter with spin-1 mediator. The cross-section from the above Lagrangian after taking all the coupling equal is given as:

$$
\sigma=\frac{1}{8 \pi\left(s-4 m_{\chi}^{2}\right)} \int_{t_{-}}^{t_{+}}|\mathcal{M}|^{2} d t
$$

where the matrix element for S-matrix and the symbol $t_{ \pm}$is given by:

$$
\begin{aligned}
|\mathcal{M}|^{2} & =\frac{n_{c} g^{4}\left(s-4 m_{\chi}^{2}\right)}{9 \pi m_{\chi}^{4} m^{2}\left(\left(s-m^{2}\right)^{2}+m^{2} \Gamma^{2}\right)}\left[2 m_{\chi}^{2} m^{4}\left(s+2 m_{f}^{2}\right)\right. \\
& \left.+2 m_{\chi}^{2}\left(s m^{4}-2 m_{f}^{2}\left(5 m^{4}-6 m^{2} s+3 s^{2}\right)\right)+3 m_{f}^{2} s\left(s-m^{2}\right)^{2}\right] \\
t_{ \pm} & =\left(m_{\chi}^{2}+m_{f}^{2}-\frac{s}{2}\right) \pm \frac{\sqrt{\left(s-4 m_{\chi}^{2}\right)\left(s-4 m_{f}^{2}\right)}}{2}
\end{aligned}
$$

where $n_{c}=3$ for quarks and 1 for leptons, $g_{A}$ and $m_{A}$ are the respective coupling and the mass of the mediator. Further taking the following approximation:

$$
s=4 m_{\chi}^{2} /\left(1+v^{2} / 4\right)
$$

we finally get the following simplified expression for the product of annihilation crosssection and velocity as:

$$
\sigma v=b v^{2}+\mathcal{O}\left(v^{3}\right)
$$

where the factor $b$ is given by:

$$
b=n_{c} g^{4} \frac{m_{\chi}^{2}}{9 \pi} \sqrt{1-\frac{m_{f}}{m_{\chi}^{2}}} \frac{4 m_{\chi}^{2}}{\left(m^{2}-4 m_{\chi}^{2}\right)^{2}+m^{2} \Gamma^{2}},
$$

and the mediator's width to SM fermions is given by:

$$
\Gamma=\frac{g^{2} n_{c} m}{6 \pi} \sqrt{1-\frac{4 m_{f}^{2}}{m^{2}}}\left[1-\frac{m_{f}^{2}}{m^{2}}\right] .
$$

The dark matter relic abundance in this case is given by:

$$
\Omega_{D M} h^{2}=\frac{1.07 \times 10^{9}}{J\left(x_{f}\right) g_{*}^{1 / 2} M_{p}}
$$

where $J\left(x_{f}\right)$ for complex vector dark matter with spin-1 mediator is given by:

$$
J\left(x_{f}\right)=\int_{x_{f}}^{\infty} n_{c} g^{4} \frac{m_{\chi}^{2}}{9 \pi} \sqrt{1-\frac{m_{f}}{m_{\chi}^{2}}} \frac{4 m_{\chi}^{2}}{\left(\left(m^{2}-4 m_{\chi}^{2}\right)^{2}+m^{2} \Gamma^{2}\right) x^{2} f_{\text {membrane }}(x)} d x .
$$


where the function $f_{\text {membrane }}(x)$ is the characteristic parameter for RS single braneworld and this can be expressed in terms of tensor-to-scalar ratio $(r)$ which is given in Eq.(3.29). Now for the GR limiting case of the $f_{\text {membrane }}(x) \rightarrow 1$ and then the relic abundance will only depend on the mass of the Dark Matter $\left(m_{\chi}\right), g_{A}$ the coupling with the spin-0 mediator and the mass of the mediator $\left(m_{A}\right)$.

$$
\Omega_{D M} h^{2}=\frac{1.07 \times 10^{9} x_{f}^{2}}{g_{*}^{1 / 2} M_{p}}\left(n_{c} g^{4} \frac{m_{\chi}^{2}}{9 \pi} \sqrt{1-\frac{m_{f}}{m_{\chi}^{2}}} \frac{4 m_{\chi}^{2}}{\left(m^{2}-4 m_{\chi}^{2}\right)^{2}+m^{2} \Gamma^{2}}\right)^{-1} .
$$

In order to constrain the coupling $\left(g_{A}\right)$ and mass $\left(m_{A}\right)$ we take the present data of the relic abundance $\left(\Omega_{D M} h^{2}=0.1199 \pm 0.0027[56]\right)$ and constrain the function $J(x)$, which in turn constrain the coupling $g_{A}$ and $m_{A}$ for a particular tensor-to-scalar ratio $(r)$. We have not shown the GR limiting case as it has been extensively been explored in [55]. In fig. (23(a)) and fig. (23(b)), we have depicted the behaviour of the effective coupling of spin-1 mediator $g_{A}$ with the varying mass the spin-1 mediator $m_{A}$ for s-channel process with three distinct value of the tensor-to-scalar ratio $r=0.001, r=0.01$ and $r=0.1$ respectively in RSII membrane. We also consider two different values of the dark matter mass $m_{\chi}=100 \mathrm{GeV}$ and $m_{\chi}=1 \mathrm{TeV}$ for the s-channel analysis. From fig. (23(a)) and fig. (23(b)), it is clearly observed that the behaviour of the effective coupling of spin-1 mediator $g_{A}$ with the varying mass the spin-1 mediator $m_{A}$ are almost similar for both of the cases and also sensitive in the vicinity of $m_{A}=2 \times 10^{2} \mathrm{GeV}$ and $m_{A}=2 \times 10^{3} \mathrm{GeV}$ respectively as it has a resonance (i.e $2 m_{\chi}=m_{A}$ ). Most importantly, in both the sides of $m_{A}=1 \times 10^{2} \mathrm{GeV}$ and $m_{A}=1 \times 10^{3} \mathrm{GeV}$ the coupling of spin-1 mediator $g_{A}$ behave in completely opposite manner.

Now for the real vector dark matter with spin-1 mediator the lagrangian is given as

$$
\mathcal{L}_{\text {membrane }} \supset\left[\frac{1}{2} g_{X}\left(X^{\mu} \partial_{\nu} X^{\nu}+\text { h.c }\right)+\bar{f} \gamma^{\mu}\left(g_{f v}+g_{f a} \gamma^{5}\right) f\right] V_{\mu}
$$

The cross-section and the thermally averaged cross section is identical to the results obtained in the context of complex scalar dark matter with spin-1 mediator case.

\subsection{Complex scalar dark matter: spin - 1/2 mediator}

The Lagrangian of complex scalar dark matter with spin $-1 / 2$ mediator is given as:

$$
\mathcal{L}_{\text {membrane }} \supset \bar{\psi}\left(\lambda_{s}+\lambda_{p} \gamma^{5}\right) f \phi^{\dagger}+\bar{f}\left(\lambda_{s}-\lambda_{p} \gamma^{5}\right) \psi \phi
$$

In fig. (24(a)), we have explicitly shown the Feynman diagramatic representation of possible $\mathrm{t} / \mathrm{u}$-channel for complex scalar dark matter with spin- $1 / 2$ mediator. 


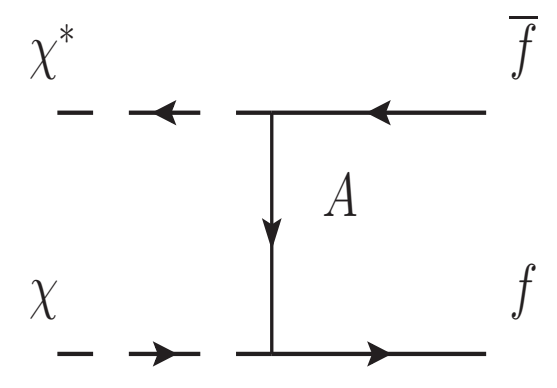

(a) $\chi \chi^{*} \rightarrow f \bar{f}$ process for $\mathrm{t} / \mathrm{u}$-channel.

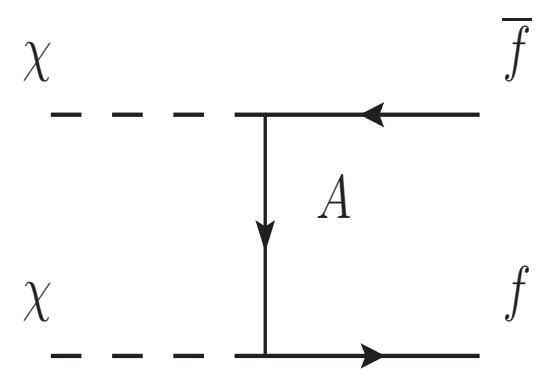

(b) $\chi_{\mu} \chi_{\nu}^{*} \rightarrow f \bar{f}$ process for $\mathrm{t} / \mathrm{u}$-channel.

Figure 24. Feynman diagrammatic representation of $\mathrm{t} / \mathrm{u}$-channel processes for complex and real scalar dark matter with spin-1/2 mediator.

The cross-section from the above Lagrangian after taking all the coupling equal is given as:

$$
\sigma=\frac{1}{8 \pi\left(s-4 m_{\chi}^{2}\right)} \int_{t_{-}}^{t_{+}}|\mathcal{M}|^{2} d t
$$

where the matrix element for the S-matrix and the symbol $t_{ \pm}$is given by:

$$
\begin{aligned}
|\mathcal{M}|^{2} & =\frac{2}{\pi s} \frac{t\left(2 m_{f}^{2}-s\right)-\left(m_{\chi}^{2}-m_{f}^{2} t\right)^{2}}{\left(t-m^{2}\right)^{2}}, \\
t_{ \pm} & =\left(m_{\chi}^{2}+m_{f}^{2}-\frac{s}{2}\right) \pm \frac{\sqrt{\left(s-4 m_{\chi}^{2}\right)\left(s-4 m_{f}^{2}\right)}}{2} .
\end{aligned}
$$

where $n_{c}=3$ for quarks and 1 for leptons, $g_{A}$ and $m_{A}$ are the respective coupling and the mass of the mediator. Further taking the following approximation:

$$
s=4 m_{\chi}^{2} /\left(1+v^{2} / 4\right)
$$

we finally get the following simplified expression for the product of annihilation crosssection and velocity as:

$$
\sigma v=a+\mathcal{O}\left(v^{2}\right)
$$

where the factor $b$ is given by:

$$
a=n_{c} g^{4} \frac{m_{f}^{2}}{4 \pi} \frac{\left[1-\frac{m_{f}}{m_{\chi}^{2}}\right]^{3 / 2}}{\left(m^{2}+m_{\chi}^{2}-m_{f}^{2}\right)} .
$$

The dark matter relic abundance in this case is given as:

$$
\Omega_{D M} h^{2}=\frac{1.07 \times 10^{9}}{J\left(x_{f}\right) g_{*}^{1 / 2} M_{p}}
$$




\section{$m_{\chi}=100 \mathrm{GeV}$}

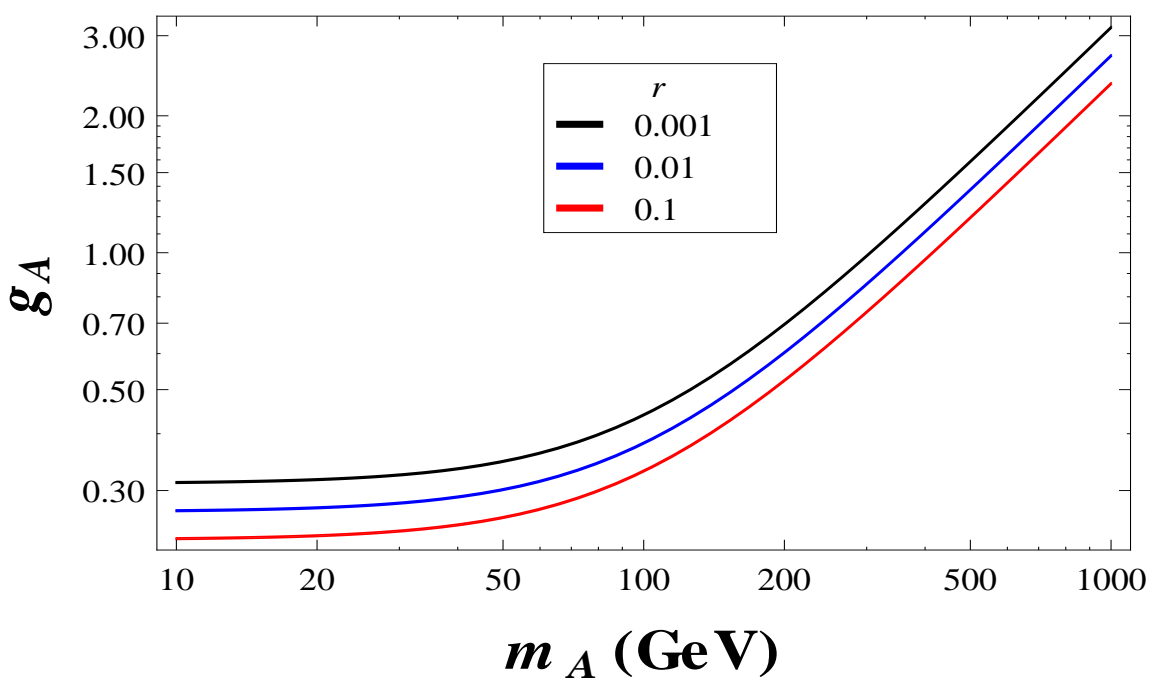

(a) $g_{A}$ vs $m_{A}$ for $\mathrm{t} / \mathrm{u}$-channel with $m_{\chi}=100 \mathrm{GeV}$.

$$
m_{\chi}=1 \mathrm{TeV}
$$

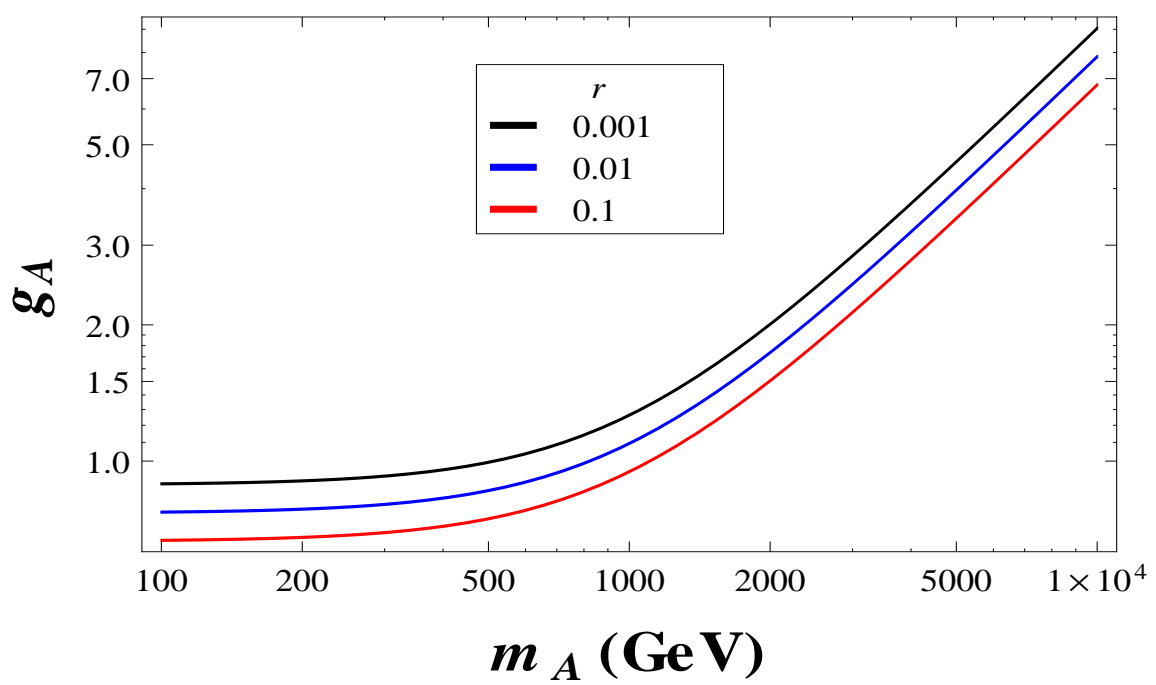

(b) $g_{A}$ vs $m_{A}$ for $\mathrm{t} / \mathrm{u}$-channel with $m_{\chi}=1 \mathrm{TeV}$.

Figure 25. In the above figure we have shown the allowed region of $g_{A}$ with respect to the mass of the mediator $m_{A}$, the above processes are $\mathrm{t} / \mathrm{u}$-channel.

where $J\left(x_{f}\right)$ for complex scalar dark matter with spin - $1 / 2$ mediator is given by:

$$
J\left(x_{f}\right)=\int_{x_{f}}^{\infty} n_{c} g^{4} \frac{m_{f}^{2}}{4 \pi} \frac{\left[1-\frac{m_{f}}{m_{\chi}^{2}}\right]^{3 / 2}}{\left(\left(m^{2}+m_{\chi}^{2}-m_{f}^{2}\right)\right) x^{2} f_{\text {membrane }}(x)} d x .
$$

where the function $f_{\text {membrane }}(x)$ is the characteristic parameter for RS single braneworld 
and this can be expressed in terms of tensor-to-scalar ratio $(r)$ which is given in Eq.(3.29). Now for the GR limiting case of the $f_{\text {membrane }}(x) \rightarrow 1$ and then the relic abundance will only depend on the mass of the Dark Matter $\left(m_{\chi}\right), g_{A}$ the coupling with the spin- 0 mediator and the mass of the mediator $\left(m_{A}\right)$.

$$
\Omega_{D M} h^{2}=\frac{1.07 \times 10^{9} x_{f}^{2}}{g_{*}^{1 / 2} M_{p}}\left(n_{c} g^{4} \frac{m_{f}^{2}}{4 \pi} \frac{\left[1-\frac{m_{f}}{m_{\chi}^{2}}\right]^{3 / 2}}{\left(m^{2}+m_{\chi}^{2}-m_{f}^{2}\right)}\right)^{-1} .
$$

In order to constrain the coupling $\left(g_{A}\right)$ and mass $\left(m_{A}\right)$ we take the present data of the relic abundance $\left(\Omega_{D M} h^{2}=0.1199 \pm 0.0027[56]\right)$ and constrain the function $J\left(x_{f}\right)$, which in turn constrain the coupling $g_{A}$ and $m_{A}$ for a particular tensor-to-scalar ratio $(r)$. We have not shown the GR limiting case as it has been extensively been explored in [55]. In fig. (25(a)) and fig. (25(b)), we have depicted the behaviour of the effective coupling of spin- $1 / 2$ mediator $g_{A}$ with the varying mass the spin-1/2 mediator $m_{A}$ for $\mathrm{t} / \mathrm{u}$-channel process with three distinct value of the tensor-to-scalar ratio $r=0.001$, $r=0.01$ and $r=0.1$ respectively in RSII membrane. We also consider two different values of the dark matter mass $m_{\chi}=100 \mathrm{GeV}$ and $m_{\chi}=1 \mathrm{TeV}$ for the t/u-channel analysis. From fig. (25(a)) and fig. (25(b)), it is clearly observed that the behaviour of the effective coupling of spin-1/2 mediator $g_{A}$ with the varying mass the spin- $1 / 2$ mediator $m_{A}$ are almost similar for both of the cases. Most importantly, in both the sides of $m_{A}=1 \times 10^{2} \mathrm{GeV}$ and $m_{A}=1 \times 10^{3} \mathrm{GeV}$ the coupling of spin- $1 / 2$ mediator $g_{A}$ behave in completely opposite manner.

\subsection{Real Scalar dark matter: spin-1/2 mediator}

The Lagrangian of real scalar dark matter with spin $-1 / 2$ mediator is given as:

$$
\mathcal{L}_{\text {membrane }} \supset \bar{\psi}\left(\lambda_{s}+\lambda_{p} \gamma^{5}\right) f \phi+\bar{f}\left(\lambda_{s}-\lambda_{p} \gamma^{5}\right) \psi \phi .
$$

The cross-section from the above Lagrangian after taking all the coupling equal is given as:

$$
\sigma=\frac{1}{8 \pi\left(s-4 m_{\chi}^{2}\right)} \int_{t_{-}}^{t_{+}}|\mathcal{M}|^{2} d t
$$

where the matrix element for the S-matrix and the symbol $t_{ \pm}$is given by:

$$
\begin{aligned}
|\mathcal{M}|^{2}=g^{4}\left[\frac{t\left(2 m_{f}^{2}-s\right)-\left(m_{\chi}^{2}-m_{f}^{2}-t\right)^{2}}{\left(t-m^{2}\right)^{2}}+\frac{u\left(2 m_{f}^{2}-s\right)-\left(m_{\chi}^{2}-m_{f}^{2}-u\right)^{2}}{\left(u-m^{2}\right)^{2}}\right. & \\
& \left.+2 \frac{\left(u-m_{\chi}^{2}+m_{f}^{2}\right)\left(m_{\chi}^{2}-m_{f}^{2}-t\right)+\left(m_{\chi}^{2}-m_{f}^{2}\right)\left(s-2 m_{f}^{2}\right)}{\left(u-m^{2}\right)\left(t-m^{2}\right)}\right], \\
t_{ \pm}= & \left(m_{\chi}^{2}+m_{f}^{2}-\frac{s}{2}\right) \pm \frac{\sqrt{\left(s-4 m_{\chi}^{2}\right)\left(s-4 m_{f}^{2}\right)}}{2}
\end{aligned}
$$




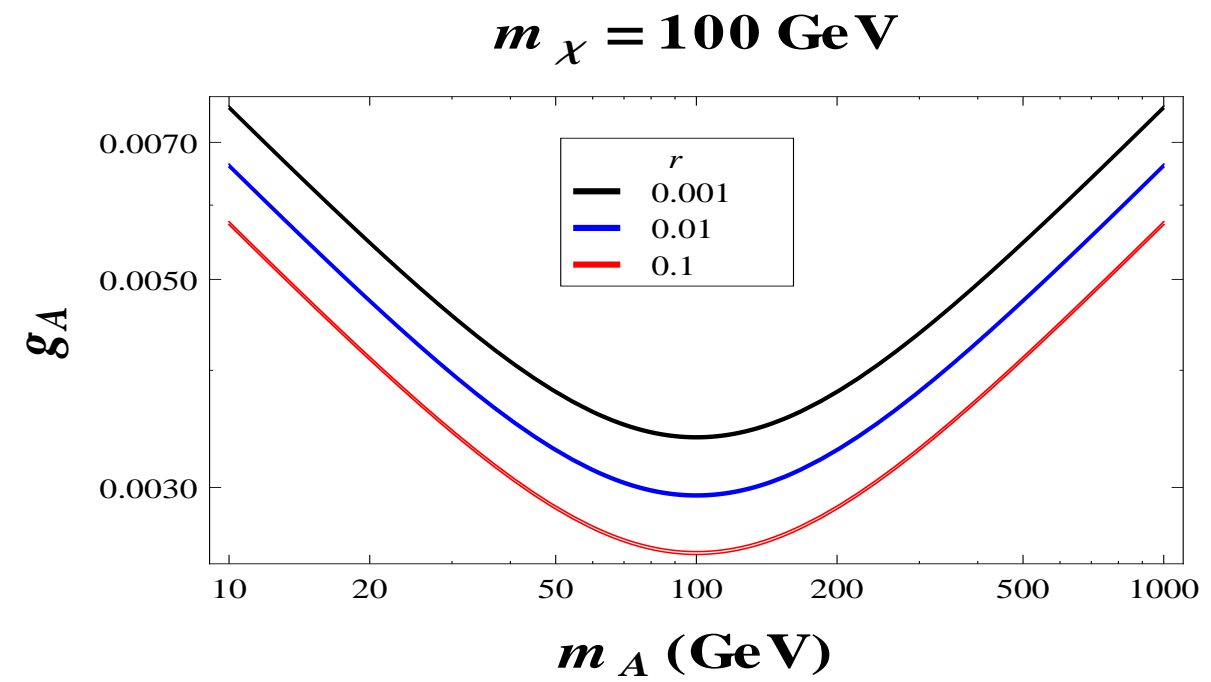

(a) $g_{A}$ vs $m_{A}$ for $\mathrm{t} / \mathrm{u}$-channel with $m_{\chi}=100 \mathrm{GeV}$.

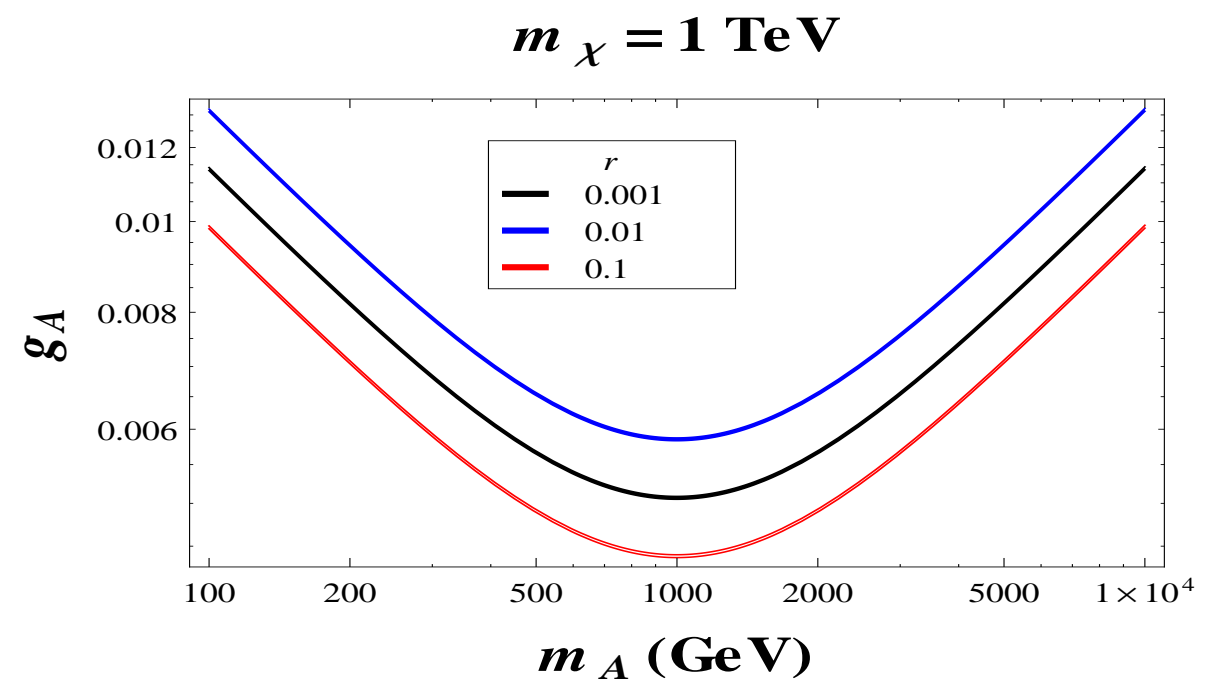

(b) $g_{A}$ vs $m_{A}$ for $\mathrm{t} / \mathrm{u}$-channel with $m_{\chi}=1 \mathrm{TeV}$.

Figure 26. In the above figure we have shown the allowed region of $g_{A}$ with respect to the mass of the mediator $m_{A}$, the above processes are $\mathrm{t} / \mathrm{u}$-channel.

where $n_{c}=3$ for quarks and 1 for leptons, $g_{A}$ and $m_{A}$ are the respective coupling and the mass of the mediator. Further taking the following approximation:

$$
s=4 m_{\chi}^{2} /\left(1+v^{2} / 4\right)
$$

we finally get the following simplified expression for the product of annihilation crosssection and velocity as:

$$
\sigma v=a+\mathcal{O}\left(v^{2}\right)
$$


where the factor $a$ is given by:

$$
a=2 n_{c} g^{4} \frac{m_{f}^{2}}{\pi} \frac{\left[1-\frac{m_{f}}{m_{\chi}^{2}}\right]^{3 / 2}}{\left(m^{2}+m_{\chi}^{2}-m_{f}^{2}\right)} .
$$

The dark matter relic abundance in this case is given as:

$$
\Omega_{D M} h^{2}=\frac{1.07 \times 10^{9}}{J\left(x_{f}\right) g_{*}^{1 / 2} M_{p}}
$$

where $J\left(x_{f}\right)$ for real scalar dark matter with spin - $1 / 2$ mediator is given by:

$$
J\left(x_{f}\right)=\int_{x_{f}}^{\infty} 2 n_{c} g^{4} \frac{m_{f}^{2}}{\pi} \frac{\left[1-\frac{m_{f}}{m_{\chi}^{2}}\right]^{3 / 2}}{\left(\left(m^{2}+m_{\chi}^{2}-m_{f}^{2}\right)\right) x^{2} f_{\text {membrane }}(x)} d x .
$$

where the function $f_{\text {membrane }}(x)$ is the characteristic parameter for RS single braneworld and this can be expressed in terms of tensor-to-scalar ratio $(r)$ which is given in Eq.(3.29). Now for the GR limiting case of the $f_{\text {membrane }}(x) \rightarrow 1$ and then the relic abundance will only depend on the mass of the Dark Matter $\left(m_{\chi}\right), g_{A}$ the coupling with the spin- 0 mediator and the mass of the mediator $\left(m_{A}\right)$.

$$
\Omega_{D M} h^{2}=\frac{1.07 \times 10^{9} x_{f}}{g_{*}^{1 / 2} M_{p}}\left(2 n_{c} g^{4} \frac{m_{f}^{2}}{\pi} \frac{\left[1-\frac{m_{f}}{m_{\chi}^{2}}\right]^{3 / 2}}{\left(m^{2}+m_{\chi}^{2}-m_{f}^{2}\right)}\right)^{-1} .
$$

In order to constrain the coupling $\left(g_{A}\right)$ and mass $\left(m_{A}\right)$ we take the present data of the relic abundance $\left(\Omega_{D M} h^{2}=0.1199 \pm 0.0027[56]\right)$ and constrain the function $J(x)$, which in turn constrain the coupling $g_{A}$ and $m_{A}$ for a particular tensor-to-scalar ratio $(r)$. We have not shown the GR limiting case as it has been extensively been explored in [55]. In fig. (26(a)) and fig. (26(b)), we have depicted the behaviour of the effective coupling of spin- $1 / 2$ mediator $g_{A}$ with the varying mass the spin-1/2 mediator $m_{A}$ for $\mathrm{t} / \mathrm{u}$-channel process with three distinct value of the tensor-to-scalar ratio $r=0.001$, $r=0.01$ and $r=0.1$ respectively in RSII membrane. We also consider three different values of the dark matter mass $m_{\chi}=100 \mathrm{GeV}$ and $m_{\chi}=1 \mathrm{TeV}$ for the t/u-channel analysis. From fig. (26(a)) and fig. (26(b)), it is clearly observed that the behaviour of the effective coupling of spin-1/2 mediator $g_{A}$ with the varying mass the spin- $1 / 2$ mediator $m_{A}$ are almost similar for both of the cases. Most importantly, in both the sides of $m_{A}=1 \times 10^{2} \mathrm{GeV}$ and $m_{A}=1 \times 10^{3} \mathrm{GeV}$ the coupling of spin- $1 / 2$ mediator $g_{A}$ behave in completely opposite manner.

\subsection{Complex Vector dark matter: spin-1/2 mediator}

The Lagrangian of real vector dark matter with spin-1/2 mediator is given as

$$
\mathcal{L}_{\text {membrane }} \supset\left[\bar{\psi} \gamma^{\mu}\left(g_{v}+g_{a} \gamma^{5}\right) f X_{\mu}^{*}+\bar{f} \gamma^{\mu}\left(g_{v}-g_{a} \gamma^{5}\right) \psi X_{\mu}\right] .
$$




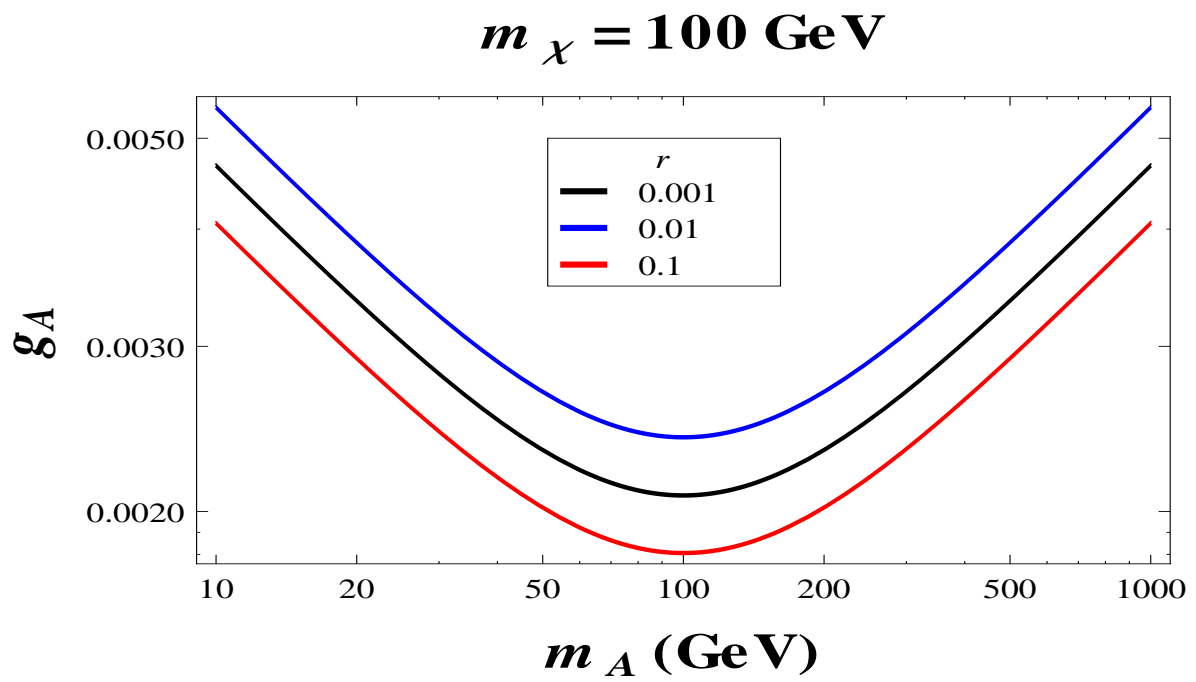

(a) $g_{A}$ vs $m_{A}$ for $\mathrm{t} / \mathrm{u}$-channel with $m_{\chi}=100 \mathrm{GeV}$.

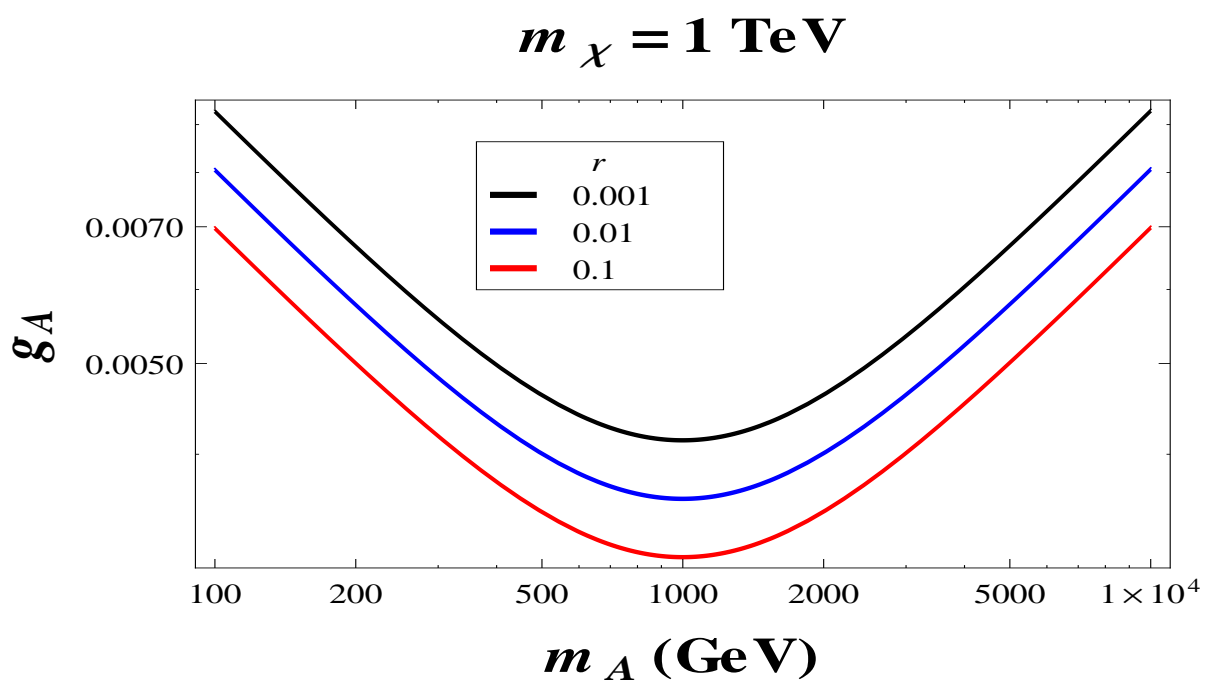

(b) $g_{A}$ vs $m_{A}$ for $\mathrm{t} / \mathrm{u}$-channel with $m_{\chi}=1 \mathrm{TeV}$.

Figure 27. In the above figure we have shown the allowed region of $g_{A}$ with respect to the mass of the mediator $m_{A}$, the above processes are $\mathrm{t} / \mathrm{u}$-channel.

The cross-section from the above Lagrangian after taking all the coupling equal is given as:

$$
\sigma=\frac{1}{8 \pi\left(s-4 m_{\chi}^{2}\right)} \int_{t_{-}}^{t_{+}}|\mathcal{M}|^{2} d t
$$


where the matrix element for the S-matrix and the symbol $t_{ \pm}$is given by:

$$
\begin{aligned}
|\mathcal{M}|^{2} & =g_{A}^{4} n_{c}\left[\frac { 1 } { m _ { \chi } ^ { 4 } ( t - m _ { A } ^ { 2 } ) ^ { 2 } } \left(m_{\chi}^{4}\left(3 m_{f}^{4}-t(4 s+5 t)\right)+2 m_{\chi}^{2}\left(t-m_{f}^{2}\right)\left(-2 t m_{f}^{2}+m_{f}^{4}+t(2 s+t)\right)\right.\right. \\
- & \left.\left(t-m_{f}^{2}\right)^{2}\left(-2 t m_{f}^{2}+m_{f}^{4}+t(s+t)\right)+4 m_{\chi}^{6}\left(m_{f}^{2}+2 t\right)-4 m_{\chi}^{8}\right) \\
& -\frac{1}{m_{\chi}^{4}\left(m_{A}^{2}-2 m_{f}^{2}-2 m_{\chi}^{2}+s+t\right)^{2}}\left(-4 m_{\chi}^{6}\left(5(s+t)-7 m_{f}^{2}\right)+m_{\chi}^{4}\left(-44 m_{f}^{2}(s+t)+29 m_{f}^{4}\right.\right. \\
& \left.+17(s+t)^{2}\right)-2 m_{\chi}^{2}\left(-3 m_{f}^{2}+2 s+3 t\right)\left(-m_{f}^{2}+s+t\right)^{2} \\
& \left.+\left(-m_{f}^{2}+s+t\right)^{2}\left(-2 t m_{f}^{2}+m_{f}^{4}+t(s+t)\right)+8 m_{\chi}^{8}\right) \\
& \left.+\frac{32\left(s-2 m_{f}^{2}\right)\left(m_{f}^{2}-m_{\chi}^{2}\right)}{\left(t-m_{A}^{2}\right)\left(m_{A}^{2}-2 m_{f}^{2}-2 m_{\chi}^{2}+s+t\right)}\right] \\
& t_{ \pm}=\left(m_{\chi}^{2}+m_{f}^{2}-\frac{s}{2}\right) \pm \frac{\sqrt{\left(s-4 m_{\chi}^{2}\right)\left(s-4 m_{f}^{2}\right)}}{2} .
\end{aligned}
$$

where $n_{c}=3$ for quarks and 1 for leptons, $g_{A}$ and $m_{A}$ are the respective coupling and the mass of the mediator. Further taking the following approximation:

$$
s=4 m_{\chi}^{2} /\left(1+v^{2} / 4\right)
$$

we finally get the following simplified expression for the product of annihilation crosssection and velocity as:

$$
\sigma v=a+\mathcal{O}\left(v^{2}\right)
$$

where the factor $a$ is given by:

$$
a \approx \frac{n_{c} g^{4} 4\left(1-m_{f}^{2} / m_{\chi}^{2}\right)^{3 / 2}}{9 \pi\left(m_{A}^{2}-m_{f}^{2}+m_{\chi}^{2}\right)^{2}}\left(32 m_{\chi}^{2}-4 m_{f}^{2}\right) .
$$

The dark matter relic abundance in this case is given as:

$$
\Omega_{D M} h^{2}=\frac{1.07 \times 10^{9}}{J\left(x_{f}\right) g_{*}^{1 / 2} M_{p}}
$$

where $J\left(x_{f}\right)$ for real scalar dark matter with spin - $1 / 2$ mediator is given by:

$$
J\left(x_{f}\right)=\int_{x_{f}}^{\infty} \frac{n_{c} g^{4} 4\left(1-m_{f}^{2} / m_{\chi}^{2}\right)^{3 / 2}}{9 \pi\left(m_{A}^{2}-m_{f}^{2}+m_{\chi}^{2}\right)^{2}} \frac{\left(32 m_{\chi}^{2}-4 m_{f}^{2}\right)}{x^{2} f_{\text {membrane }}(x)} d x .
$$

where the function $f_{\text {membrane }}(x)$ is the characteristic parameter for RS single braneworld and this can be expressed in terms of tensor-to-scalar ratio (r) which is given in Eq.(3.29). Now for the GR limiting case of the $f_{\text {membrane }}(x) \rightarrow 1$ and then the relic 
abundance will only depend on the mass of the Dark Matter $\left(m_{\chi}\right), g_{A}$ the coupling with the spin- 0 mediator and the mass of the mediator $\left(m_{A}\right)$.

$$
\Omega_{D M} h^{2}=\frac{1.07 \times 10^{9} x_{f}}{g_{*}^{1 / 2} M_{p}}\left(\frac{n_{c} g^{4} 4\left(1-m_{f}^{2} / m_{\chi}^{2}\right)^{3 / 2}}{9 \pi\left(m_{A}^{2}-m_{f}^{2}+m_{\chi}^{2}\right)^{2}}\left(32 m_{\chi}^{2}-4 m_{f}^{2}\right)\right)^{-1}
$$

In order to constrain the coupling $\left(g_{A}\right)$ and mass $\left(m_{A}\right)$ we take the present data of the relic abundance $\left(\Omega_{D M} h^{2}=0.1199 \pm 0.0027[56]\right)$ and constrain the function $J\left(x_{f}\right)$, which in turn constrain the coupling $g_{A}$ and $m_{A}$ for a particular tensor-toscalar ratio $(r)$. We have not shown the GR limiting case as it has been extensively been explored in [55].

In fig. (27(a)) and fig. (27(b)), we have depicted the behaviour of the effective coupling of spin- $1 / 2$ mediator $g_{A}$ with the varying mass the spin-1/2 mediator $m_{A}$ for $\mathrm{t} / \mathrm{u}$-channel process with three distinct value of the tensor-to-scalar ratio $r=0.001$, $r=0.01$ and $r=0.1$ respectively in RSII membrane. We also consider two different values of the dark matter mass $m_{\chi}=100 \mathrm{GeV}$ and $m_{\chi}=1 \mathrm{TeV}$ for the $\mathrm{t} / \mathrm{u}$-channel analysis. From fig. (27(a)) and fig. (27(b)), it is clearly observed that the behaviour of the effective coupling of spin-1/2 mediator $g_{A}$ with the varying mass the spin- $1 / 2$ mediator $m_{A}$ are almost similar for both of the cases. Most importantly, in both the sides of $m_{A}=1 \times 10^{2} \mathrm{GeV}$ and $m_{A}=1 \times 10^{3} \mathrm{GeV}$ the coupling of spin- $1 / 2$ mediator $g_{A}$ behave in completely opposite manner.

Now, for the real vector dark matter with spin- $1 / 2$ mediator the Lagrangian of is given as

$$
\mathcal{L}_{\text {membrane }} \supset\left[\bar{\psi} \gamma^{\mu}\left(g_{v}+g_{a} \gamma^{5}\right) f X_{\mu}+\bar{f} \gamma^{\mu}\left(g_{v}-g_{a} \gamma^{5}\right) \psi X_{\mu}\right]
$$

The cross-section and the thermally averaged cross section is almost identical to the results obtained in the context of complex scalar dark matter with spin-1/2 mediator case.

\section{Summary}

To summarize, in the present article, we have addressed the following points:

- We have established a theoretical constraint relationship to explicitly show a direct connection between the dark matter relic abundance $\left(\Omega_{D M} h^{2}\right)$ and primordial gravity waves $(r)$, which establish a precise connection between inflation and generation of dark matter within the framework of effective field theory in RSII membrane paradigm. In fig. (1), we have shown the algorithm of the prescribed methodology proposed in this paper. Also in fig. (2), we have depicted the model independent inflationary constraint from the primordial gravitational waves via tensor-to-scalar ratio on the dark matter relic 
abundance within the framework of effective field theory of RSII membrane paradigm. From fig. (2), it is clearly observed that for $r<0.01$, various inflationary models in membrane paradigm are in huge tension with the Planck 2015 constraint on dark matter relic abundance. We have also shown the the dependence of the relic abundance with $\alpha$ (defined in eq (3.12)) in fig. (3) for various tensor-scalar ratio $r$. Obviously the result changes for different thermally averaged cross-section $\langle\sigma v\rangle$, but one may note the in direct relation of the tensor to scalar ratio to the model parameters which gives rise to the $\langle\sigma v\rangle$. On the other hand, for $0.01 \leq r \leq 0.12$, various inflationary models in membrane paradigm are consistent with $2 \sigma$ constraint on dark matter relic abundance obtained from Planck 2015 data. In fig. (4(a)) and fig. (4(b)), we have depicted the model independent inflationary constraint from the primordial gravitational waves via tensor-to-scalar ratio on the thermally averaged annihilation crosssection of dark matter content for the dark matter mass, $m_{\chi}=100 \mathrm{GeV}$ and $m_{\chi}=1 \mathrm{TeV}$ respectively, within the framework of effective field theory of RSII membrane paradigm. Here it is clearly observed that the value of the inflationary tensor-to-scalar ratio decreases with the increase in the thermally averaged annihilation cross-section of dark matter content. Also it is important to note that for of these three cases within the allowed range of tensor-to-scalar ratio, $0.01 \leq r \leq 0.12$, thermally averaged annihilation cross-section of dark matter content is constrained within the window, $\langle\sigma v\rangle \sim \mathcal{O}\left(10^{-28}-10^{-27}\right) \mathrm{cm}^{3} / \mathrm{s}$.

- We have explicitly studied the details of Effective Field Theory of dark matter from membrane paradigm paradigm. We have explicitly studied:

- the $s$ and $t / u$ channel interaction of the Dirac dark matter with spin-0 and spin-1 mediator, Majorana dark matter with spin-1 mediator.

- After that, we have studied the consequences from s-channel interaction of complex and real scalar dark matter with spin-0 mediator, complex scalar and real vector dark matter with spin-1 mediator, complex and real vector dark matter with spin-0 and spin-1 mediator respectively.

- Finally, we have studied the consequences from t/u-channel interaction of complex and real scalar and complex and real vector dark matter with spin- $1 / 2$ mediator.

- In most of the case we have not gone to higher Dark Matter masses (i.e $\mathcal{O}(10 \mathrm{TeV})$ in some cases $\mathcal{O}(\mathrm{TeV}))$ as the couplings were exceeding $\mathcal{O}(1)$ violating perturbative limit.

- Most significantly, once the signature of primordial gravity waves will be predicted by in any near future observational probes, it will be possible to put 
further stringent constraint on the dark matter abundance from our derived result.

- In this paper, we have used important cosmological and particle physics constraints arising from Planck 2015 and Planck+BICEP2/Keck Array joint data on the the upper bound on tenor to scalar ratio and the bound on the dark matter abundance within $1.5 \sigma-2 \sigma$ statistical CL.

Further our aim is to carry forward this work in a more broader sense, where we will apply all the derived results to constrain the inflationary observables and cosmological parameters obtained from various models of membrane inflation. The other promising future prospects of this work are:-

1. One can follow the prescribed methodology to derive the cosmological constraints in the context of various modified gravity framework i.e. Dvali-GabadadzePorrati (DGP) braneworld [57], Einstein-Hilbert-Gauss-Bonnet (EHGB) gravity [41, 58-60, 62-64], Einstein-Gauss-Bonnet-Dilaton (EGBD) gravity [59, 60, $62-64]$ and $f(R)$ theory of gravity $[65,66]$ etc.

2. Hence using the derived constraints one can further constrain various classes of (membrane) inflationary models within the framework of other modified theories of gravity.

3. One can explore the details of UV completion in the context of RSII membrane and for other versions of extended theories of gravity.

4. Detailed study of the collider constraints on the effective theory prescription from extended theories of gravity is one of the promising and unexplored areas in this context.

5. Generation of scalar dark matter and detailed study of the constraints from the UV complete extended theory of gravity is also an important issue, which we will explore very soon in our follow up work.

6. One can also implement the methodology for the alternative theories of inflation i.e. bouncing frameworks and related ideas. For an example one can investigate for the cosmological implications of cosmic hysteresis scenario [67-72] in the generation of dark matter.

7. Explaining the origin of dark matter in presence of non-standard/ non-canonical kinetic term, using non-minimal inflaton coupling to gravity sector [73], multifield sector and also exploring the highly non-linear regime of effective field theory are open issues in this literature. String theory originated DBI and tachyonic inflationary frameworks $[41,58,74]$ are the two prominent and well 
known examples of non-standard field theoretic setup through which one can explore various open questions in this area.

8. One can also study the connecting relation between dark matter abundance and primordial gravity waves with the inflationary magnetogenesis and standard leptogenesis scenario [75] from the relevant effective field theory operators. In the context of RSII single membrane we have recently studied some of these issues elaborately [37].

\section{Acknowledgments}

SC would like to thank Department of Theoretical Physics, Tata Institute of Fundamental Research, Mumbai for providing me Visiting (Post-Doctoral) Research Fellowship. SC take this opportunity to thank sincerely to Sandip P. Trivedi, Shiraz Minwalla, Soumitra SenGupta, Sudhakar Panda, Varun Sahni, Sayan Kar and Supratik Pal for their constant support and inspiration. SC take this opportunity to thank all the active members and the regular participants of weekly student discussion meet COSMOMEET from Department of Theoretical Physics and Department of Astronomy and Astrophysics, Tata Institute of Fundamental Research for their strong support. SC also thank Sandip Trivedi and Shiraz Minwalla for giving the opportunity to be the part of String Theory and Mathematical Physics Group. SC also thank the other post-docs and doctoral students from String Theory and Mathematical Physics Group for providing an excellant academic ambience during the research work. SC additionally take this opportunity to thank the organizers of STRINGS, 2015, International Centre for Theoretical Science, Tata Institute of Fundamental Research (ICTS,TIFR), Indian Institute of Science (IISC) and specially Shiraz Minwalla for giving the opportunity to participate in STRINGS, 2015 and also providing the local hospitality during the work. SC also thank the organizers of National String Meet 2015 and International Conference on Gravitation and Cosmology, IISER, Mohali and COSMOASTRO 2015, Institute of Physics for providing the local hospitality during the work. SC also thanks the organizers of School and Workshop on Large Scale Structure: From Galaxies to Cosmic Web, The Inter-University Centre for Astronomy and Astrophysics (IUCAA), Pune, India and specially Aseem Paranjape and Varun Sahni for providing the academic visit during the work. Last but not the least, I would all like to acknowledge our debt to the people of India for their generous and steady support for research in natural sciences, especially for theoretical high energy physics, string theory and cosmology. 


\section{Appendix}

\section{A. Consistency relations in RSII membrane paradigm}

In the context of RSII the spectral tilts $\left(n_{S}, n_{T}\right)$, running of the tilts $\left(\alpha_{S}, \alpha_{T}\right)$ and running of the running of tilts $\left(\kappa_{T}, \kappa_{S}\right)$ at the momentum pivot scale $k_{*}$ can be expressed as:

$$
\begin{aligned}
n_{S}\left(k_{*}\right)-1 & =2 \eta_{b}\left(\phi_{*}\right)-6 \epsilon_{b}\left(k_{*}\right) \\
n_{T}\left(k_{*}\right) & =-3 \epsilon_{b}\left(k_{*}\right)=-\frac{r\left(k_{*}\right)}{8} \\
\alpha_{S}\left(k_{*}\right) & =16 \eta_{b}\left(k_{*}\right) \epsilon_{b}\left(k_{*}\right)-18 \epsilon_{b}^{2}\left(k_{*}\right)-2 \xi_{b}^{2}\left(k_{*}\right) \\
\alpha_{T}\left(k_{*}\right)= & 6 \eta_{b}\left(k_{*}\right) \epsilon_{b}\left(k_{*}\right)-9 \epsilon_{b}^{2}\left(k_{*}\right) \\
\kappa_{S}\left(k_{*}\right)= & 152 \eta_{b}\left(k_{*}\right) \epsilon_{b}^{2}\left(k_{*}\right)-32 \epsilon_{b}\left(k_{*}\right) \eta_{b}^{2}\left(k_{*}\right)-108 \epsilon_{b}^{3}\left(k_{*}\right) \\
& \quad-24 \xi_{b}^{2}\left(k_{*}\right) \epsilon_{b}\left(k_{*}\right)+2 \eta_{b}\left(k_{*}\right) \xi_{b}^{2}\left(k_{*}\right)+2 \sigma_{b}^{3}\left(k_{*}\right) \\
\kappa_{T}\left(k_{*}\right)= & 66 \eta_{b}\left(k_{*}\right) \epsilon_{b}^{2}\left(k_{*}\right)-12 \epsilon_{b}\left(k_{*}\right) \eta_{b}^{2}\left(k_{*}\right)-54 \epsilon_{b}^{3}\left(k_{*}\right)-6 \epsilon_{b}\left(k_{*}\right) \xi_{b}^{2}\left(k_{*}\right) .
\end{aligned}
$$

In terms of slow-roll parameters in RSII setup one can also write the following sets of consistency conditions for membrane inflation:

$$
\begin{aligned}
n_{T}\left(k_{*}\right)-n_{S}\left(k_{*}\right)+1= & \left(\frac{d \ln r(k)}{d \ln k}\right)_{*}=\left[\frac{r\left(k_{*}\right)}{8}-2 \eta_{b}\left(k_{*}\right)\right] \\
\alpha_{T}\left(k_{*}\right)-\alpha_{S}\left(k_{*}\right)= & \left(\frac{d^{2} \ln r(k)}{d \ln k^{2}}\right)_{*}=\left[\left(\frac{r\left(k_{*}\right)}{8}\right)^{2}-\frac{20}{3}\left(\frac{r\left(k_{*}\right)}{8}\right)+2 \xi_{b}^{2}\left(k_{*}\right)\right], \quad(5.8) \\
\kappa_{T}\left(k_{*}\right)-\kappa_{S}\left(k_{*}\right)= & \left(\frac{d^{3} \ln r(k)}{\left.d \ln k^{3}\right)_{*}}=\right. \\
= & {\left[\frac{r\left(k_{*}\right)}{8}\right)^{3}-\frac{86}{9}\left(\frac{r\left(k_{*}\right)}{8}\right)^{2} } \\
& \left.+\frac{4}{3}\left(6 \xi_{b}^{2}\left(k_{*}\right)+5 \eta_{b}^{2}\left(k_{*}\right)\right)\left(\frac{r\left(k_{*}\right)}{8}\right)+2 \eta_{b}\left(k_{*}\right) \xi_{b}^{2}\left(k_{*}\right)+2 \sigma_{b}^{3}\left(k_{*}\right)\right] .
\end{aligned}
$$

Here Eq (5.7-5.9)) represent the running, running of the running and running of the double running of tensor-to-scalar ratio in RSII membrane inflationary setup. In this section let us In the high energy limit $\rho>>\sigma$, Eq (2.21) is written using the slow-roll approximation as:

$$
H^{2} \approx \frac{\rho^{2}}{6 M_{p}^{2} \sigma} \approx \frac{V^{2}(\phi)}{6 M_{p}^{2} \sigma},
$$


where $V(\phi)$ be the inflaton single field potential. Within high energy limit $\rho>>\sigma$ the slow-roll parameters in the visible membrane can be expressed as:

$$
\begin{aligned}
\epsilon_{b}(\phi) & \approx \frac{2 M_{p}^{2} \sigma\left(V^{\prime}(\phi)\right)^{2}}{V^{3}(\phi)}, \\
\eta_{b}(\phi) & \approx \frac{2 M_{p}^{2} \sigma V^{\prime \prime}(\phi)}{V^{2}(\phi)}, \\
\xi_{b}^{2}(\phi) & \approx \frac{4 M_{p}^{4} \sigma^{2} V^{\prime}(\phi) V^{\prime \prime \prime}(\phi)}{V^{4}(\phi)}, \\
\sigma_{b}^{3}(\phi) & \approx \frac{8 M_{p}^{6} \sigma^{3}\left(V^{\prime}(\phi)\right)^{2} V^{\prime \prime \prime \prime}(\phi)}{V^{6}(\phi)} .
\end{aligned}
$$

and consequently the number of e-foldings can be written as:

$$
\Delta \mathcal{N}_{b}=\mathcal{N}_{b}\left(\phi_{c m b}\right)-\mathcal{N}_{b}\left(\phi_{e}\right) \approx \frac{1}{2 \sigma M_{p}^{2}} \int_{\phi_{e}}^{\phi_{c m b}} d \phi \frac{V^{2}(\phi)}{V^{\prime}(\phi)}
$$

where $\phi_{e}$ corresponds to the field value at the end of inflation, which can be obtained from the following constraint equation:

$$
\max _{\phi=\phi_{e}}\left[\epsilon_{b},\left|\eta_{b}\right|,\left|\xi_{b}^{2}\right|,\left|\sigma_{b}^{3}\right|\right]=1
$$

\section{B. Relic Abundance Results for GR limitng case}

It is important to note that, for Standard General Relativistic (GR) prescription the Boltzmann equation for the dark matter relic abundance is given as follows:

$$
\frac{d Y_{\mathrm{DM}}}{d \Theta}=\frac{s\langle\sigma v\rangle}{H \Theta}\left[1+\frac{1}{3} \frac{d \ln g_{s}}{d \ln \Theta}\right]\left[\left(Y_{\mathrm{DM}}^{E Q}\right)^{2}-Y_{\mathrm{DM}}^{2}\right]
$$

where the solution for the dark matter relic abundance $Y_{\mathrm{DM}}^{E Q}(\Theta)$ is given as:

$$
Y_{\mathrm{DM}}^{E Q}(\Theta)=\frac{n_{\mathrm{DM}}^{E Q}}{s}=\frac{45}{2 \pi^{4}}\left(\frac{\pi}{8}\right)^{1 / 2} \frac{g_{\mathrm{DM}}}{g_{*}} \Theta^{3 / 2} e^{-\Theta}
$$

Here $s$ is the entropy density as defined in Eq (3.20), $g_{\mathrm{DM}}$ signifies the effective number of degrees of freedom corresponding to the dark matter content and for non-relativistic case the thermally averaged cross-section can be expressed as:

$$
\langle\sigma v\rangle \approx\langle\sigma v\rangle_{N R}=a+\frac{3}{2} \frac{b}{\Theta}+\frac{15}{8} \frac{c}{\Theta^{2}}+\frac{35}{16} \frac{d}{\Theta^{3}}+\frac{315}{128} \frac{e}{\Theta^{4}}+\cdots .
$$

For numerical estimation we will restrict ourselves up to the second term in the above series expansion of $\langle\sigma v\rangle_{N R}$. In more technical language this is commonly known as s-wave approximation. See the ref. [54], in which all the derivation have done in detail. Also it is important to mention here that, in a very specific physical situation 
when the energy density of the dark matter content is very very small compared to the membrane tension in RSII membrane paradigm $\left(\rho_{D M}=\rho_{\chi}<<\sigma\right)$, then one can can reproduce all the known results for standard GR. On the other hand, the explicit role of RSII membarne paradigm can be clearly visualized when the energy density of the dark matter content is very very large compared to the membrane tension in RSII membrane paradigm $\left(\rho_{D M}=\rho_{\chi}>>\sigma\right)$.

Now from the equation eq.(3.23),(3.24) and (3.25) for the standard GR case all three functions $\langle\widetilde{\sigma} v\rangle(\Theta), \Sigma(\Theta)$ and characteristic function, $f_{\text {brane }}(\Theta)$ reduced to the following simplified expressions:

$$
\begin{aligned}
\langle\widetilde{\sigma} v\rangle(\Theta) & =\langle\sigma v\rangle, \\
\Sigma(\Theta) & =\sqrt{\frac{g_{*}}{90}} \frac{\pi m_{\chi}^{2}}{M_{p} \Theta^{2}}=H_{G R}(\Theta), \\
f_{\text {membrane }}(\Theta) & =1,
\end{aligned}
$$

where $H_{G R}(\Theta)$ is the Hubble parameter in GR.

where $\Theta_{F}$ is the freeze-out temperature which can be calculated by numerically solving the following transcendental equation as:

$$
\Theta_{F}=\ln \left(\frac{0.038 g_{\mathrm{DM}} m_{\chi} M_{p}\langle\sigma v\rangle(\Theta)}{g_{*}^{1 / 2} \Theta_{F}^{1 / 2}}\right) .
$$

Now, the dark matter relic abundance can be found out by

$$
\Omega_{\mathrm{DM}} h^{2}=\left(\frac{m_{\chi} s Y_{\mathrm{DM}}}{3 H^{2} M_{p}^{2}}\right)_{\text {today }} h^{2} \simeq \frac{1.07 \times 10^{9} \mathrm{GeV}^{-1}}{J\left(\Theta_{F}\right) g_{*}^{1 / 2} M_{p}}
$$

where using s-wave approximation and for the context of standard GR the integral $J\left(\Theta_{F}\right)$ can be computed as:

$$
\begin{aligned}
J\left(\Theta_{F}\right) & =\int_{\Theta_{F}}^{\infty} d \Theta \frac{\langle\sigma v\rangle(\Theta)}{\Theta^{2}} \\
& \approx \int_{\Theta_{F}}^{\infty} d \Theta \int_{0}^{\infty} d v \frac{v^{2}\left(a+b v^{2}\right)}{\sqrt{4 \pi \Theta}} e^{-\Theta v^{2} / 4} \\
& =\int_{\Theta_{F}}^{\infty}\left(a+\frac{3}{2} \frac{b}{\Theta}\right) \frac{d \Theta}{\Theta^{2}}=\frac{1}{\Theta_{F}}\left(a+\frac{3 b}{4 \Theta_{F}}\right) .
\end{aligned}
$$

In the context of GR, dark matter relic abundance can be expressed as:

$$
\Omega_{\mathrm{DM}} h^{2} \simeq \frac{1.07 \times 10^{9} \Theta_{F} \mathrm{GeV}^{-1}}{g_{*}^{1 / 2} M_{p}\left(a+\frac{3 b}{4 \Theta_{F}}\right)}
$$

It is important to note that, in the vicinity of resonance the Taylor series expansion breaks down and in such a case it is legitimate to consider the numerical solution of 
$J\left(\Theta_{F}\right)$, which is introduced in Eq (3.27). Additionally, it is mention here that in our computation we treat the integral $J\left(\Theta_{F}\right)$ numerically to obtain the accurate result in the context of membrane paradigm. Here from Eq (5.26), it is clearly observed that the dark matter relic abundance cannot be directly related to the tensor-toscalar ratio $(r)$ in GR limit using complete model independent prescription. If we specifically mention the model to describe the decay process of inflaton to dark matter then it is possible to obtain a connecting relationship between the dark matter relic abundance and tensor-to-scalar ratio in a model dependent fashion. See ref. ([76]) for further details.

\section{References}

[1] M. Cirelli, "Indirect Searches for Dark Matter: a status review," Pramana 79 (2012) 1021 [arXiv:1202.1454 [hep-ph]].

[2] L. Baudis, "Direct dark matter detection: the next decade," Phys. Dark Univ. 1 (2012) 94 [arXiv:1211.7222 [astro-ph.IM]].

[3] G. Aad et al. [ATLAS Collaboration], "Search for dark matter candidates and large extra dimensions in events with a jet and missing transverse momentum with the ATLAS detector,” JHEP 1304 (2013) 075 [arXiv:1210.4491 [hep-ex]].

[4] S. Chatrchyan et al. [CMS Collaboration], "Search for New Physics with a Mono-Jet and Missing Transverse Energy in pp Collisions at $\sqrt{s}=7$ TeV," Phys. Rev. Lett. 107 (2011) 201804 [arXiv:1106.4775 [hep-ex]].

[5] [ATLAS Collaboration], "Search for New Phenomena in Monojet plus Missing Transverse Momentum Final States using 10fb-1 of pp Collisions at sqrts $=8 \mathrm{TeV}$ with the ATLAS detector at the LHC," ATLAS-CONF-2012-147.

[6] [CMS Collaboration], "Search for new physics in monojet events in pp collisions at $\operatorname{sqrt}(s)=8 \mathrm{TeV}, "$ CMS-PAS-EXO-12-048.

[7] G. Aad et al. [ATLAS Collaboration], "Search for dark matter candidates and large extra dimensions in events with a photon and missing transverse momentum in $p p$ collision data at $\sqrt{s}=7$ TeV with the ATLAS detector," Phys. Rev. Lett. 110 (2013) 1, 011802 [arXiv:1209.4625 [hep-ex]].

[8] S. Chatrchyan et al. [CMS Collaboration], "Search for Dark Matter and Large Extra Dimensions in pp Collisions Yielding a Photon and Missing Transverse Energy," Phys. Rev. Lett. 108 (2012) 261803 [arXiv:1204.0821 [hep-ex]].

[9] [ATLAS Collaboration], "Search for dark matter candidates and large extra dimensions in events with a photon and missing transverse momentum in pp collision data at sqrt $(s)=7 \mathrm{TeV}$ with the ATLAS detector," ATLAS-CONF-2012-085.

[10] CMS Collaboration [CMS Collaboration], "Search for ADD Extra-dimensions in Monophotons," CMS-PAS-EXO-11-058. 
[11] G. Busoni, A. De Simone, E. Morgante and A. Riotto, "On the Validity of the Effective Field Theory for Dark Matter Searches at the LHC," Phys. Lett. B 728 (2014) 412 [arXiv:1307.2253 [hep-ph]].

[12] G. Busoni, A. De Simone, J. Gramling, E. Morgante and A. Riotto, "On the Validity of the Effective Field Theory for Dark Matter Searches at the LHC, Part II: Complete Analysis for the s-channel," JCAP 1406 (2014) 060 [arXiv:1402.1275 [hep-ph]].

[13] G. Busoni, A. De Simone, T. Jacques, E. Morgante and A. Riotto, "On the Validity of the Effective Field Theory for Dark Matter Searches at the LHC Part III: Analysis for the t-channel," JCAP 1409 (2014) 022 [arXiv:1405.3101 [hep-ph]].

[14] C. P. Burgess, "Introduction to Effective Field Theory," Ann. Rev. Nucl. Part. Sci. 57 (2007) 329 [hep-th/0701053].

[15] R. Sundrum, "Effective field theory for a three-brane universe," Phys. Rev. D 59 (1999) 085009 [hep-ph/9805471].

[16] C. Cheung, P. Creminelli, A. L. Fitzpatrick, J. Kaplan and L. Senatore, "The Effective Field Theory of Inflation,” JHEP 0803 (2008) 014 [arXiv:0709.0293 [hep-th]].

[17] S. Weinberg, "Effective Field Theory for Inflation," Phys. Rev. D 77 (2008) 123541 [arXiv:0804.4291 [hep-th]].

[18] L. Senatore and M. Zaldarriaga, "The Effective Field Theory of Multifield Inflation," JHEP 1204 (2012) 024 [arXiv:1009.2093 [hep-th]].

[19] D. Lopez Nacir, R. A. Porto, L. Senatore and M. Zaldarriaga, "Dissipative effects in the Effective Field Theory of Inflation," JHEP 1201 (2012) 075 [arXiv:1109.4192 [hep-th]].

[20] M. Beltran, D. Hooper, E. W. Kolb, Z. A. C. Krusberg and T. M. P. Tait, "Maverick dark matter at colliders," JHEP 1009 (2010) 037 [arXiv:1002.4137 [hep-ph]].

[21] M. Duch, B. Grzadkowski and J. Wudka, "Classification of effective operators for interactions between the Standard Model and dark matter," JHEP 1505, 116 (2015) [arXiv:1412.0520 [hep-ph]].

[22] M. Duch, "Effective Operators for Dark Matter Interactions," arXiv:1410.4427 [hep-th].

[23] M. B. Krauss, S. Morisi, W. Porod and W. Winter, "Higher Dimensional Effective Operators for Direct Dark Matter Detection," JHEP 1402, 056 (2014) [arXiv:1312.0009 [hep-ph]].

[24] N. F. Bell, Y. Cai and A. D. Medina, "Co-annihilating Dark Matter: Effective Operator Analysis and Collider Phenomenology," Phys. Rev. D 89, no. 11, 115001 (2014) [arXiv:1311.6169 [hep-ph]].

[25] A. De Simone, A. Monin, A. Thamm and A. Urbano, "On the effective operators for Dark Matter annihilations," JCAP 1302, 039 (2013) [arXiv:1301.1486 [hep-ph]]. 
[26] Y. J. Chae and M. Perelstein, "Dark Matter Search at a Linear Collider: Effective Operator Approach," JHEP 1305, 138 (2013) [arXiv:1211.4008 [hep-ph]].

[27] J. Abdallah et al., "Simplified Models for Dark Matter Searches at the LHC," Phys. Dark Univ. 9-10, 8 [arXiv:1506.03116 [hep-ph]].

[28] G. Busoni, A. De Simone, T. Jacques, E. Morgante and A. Riotto, "Making the Most of the Relic Density for Dark Matter Searches at the LHC 14 TeV Run," JCAP 1503, no. 03, 022 (2015) [arXiv:1410.7409 [hep-ph]].

[29] J. Goodman, M. Ibe, A. Rajaraman, W. Shepherd, T. M. P. Tait and H. B. Yu, "Constraints on Light Majorana dark Matter from Colliders," Phys. Lett. B 695 (2011) 185 [arXiv:1005.1286 [hep-ph]].

[30] Y. Bai, P. J. Fox and R. Harnik, "The Tevatron at the Frontier of Dark Matter Direct Detection," JHEP 1012 (2010) 048 [arXiv:1005.3797 [hep-ph]].

[31] J. Goodman, M. Ibe, A. Rajaraman, W. Shepherd, T. M. P. Tait and H. B. Yu, "Constraints on Dark Matter from Colliders," Phys. Rev. D 82 (2010) 116010 [arXiv:1008.1783 [hep-ph]].

[32] A. Rajaraman, W. Shepherd, T. M. P. Tait and A. M. Wijangco, "LHC Bounds on Interactions of Dark Matter," Phys. Rev. D 84 (2011) 095013 [arXiv:1108.1196 [hep-ph]].

[33] P. J. Fox, R. Harnik, J. Kopp and Y. Tsai, "Missing Energy Signatures of Dark Matter at the LHC," Phys. Rev. D 85 (2012) 056011 [arXiv:1109.4398 [hep-ph]].

[34] S. Choudhury, "Can Effective Field Theory of inflation generate large tensor-to-scalar ratio within RandallSundrum single braneworld?," Nucl. Phys. B 894 (2015) 29 [arXiv:1406.7618 [hep-th]].

[35] D. Baumann and L. McAllister, "Inflation and String Theory," arXiv:1404.2601 [hep-th].

[36] V. Assassi, D. Baumann, D. Green and L. McAllister, "Planck-Suppressed Operators," JCAP 1401 (2014) 033 [arXiv:1304.5226 [hep-th]].

[37] S. Choudhury, "Constraining brane inflationary magnetic field from cosmoparticle physics after Planck," JHEP 1510 (2015) 095 [arXiv:1504.08206 [astro-ph.CO]].

[38] S. Choudhury and S. Pal, "Brane inflation in background supergravity," Phys. Rev. D 85 (2012) 043529 [arXiv:1102.4206 [hep-th]].

[39] S. Choudhury and S. Pal, "Reheating and leptogenesis in a SUGRA inspired brane inflation," Nucl. Phys. B 857 (2012) 85 [arXiv:1108.5676 [hep-ph]].

[40] S. Choudhury and S. Pal, "Brane inflation: A field theory approach in background supergravity,” J. Phys. Conf. Ser. 405 (2012) 012009 [arXiv:1209.5883 [hep-th]].

[41] S. Choudhury and S. Pal, "DBI Galileon inflation in background SUGRA," Nucl. Phys. B 874 (2013) 85 [arXiv:1208.4433 [hep-th]]. 
[42] T. Shiromizu, K. i. Maeda and M. Sasaki, "The Einstein equation on the 3-brane world," Phys. Rev. D 62 (2000) 024012 [gr-qc/9910076].

[43] L. Randall and R. Sundrum, "An Alternative to compactification," Phys. Rev. Lett. 83 (1999) 4690 [hep-th/9906064].

[44] R. Maartens and K. Koyama, "Brane-World Gravity," Living Rev. Rel. 13 (2010) 5 [arXiv:1004.3962 [hep-th]].

[45] P. Brax, C. van de Bruck and A. C. Davis, "Brane world cosmology," Rept. Prog. Phys. 67 (2004) 2183 [hep-th/0404011].

[46] S. Choudhury, "Reconstructing inflationary paradigm within Effective Field Theory framework," Phys. Dark Univ. 11 (2016) 16 [arXiv:1508.00269 [astro-ph.CO]].

[47] S. Choudhury and A. Mazumdar, "Sub-Planckian inflation $\&$ large tensor to scalar ratio with $r \geq 0.1$," arXiv:1404.3398 [hep-th].

[48] S. Choudhury and A. Mazumdar, "Reconstructing inflationary potential from BICEP2 and running of tensor modes," arXiv:1403.5549 [hep-th].

[49] S. Choudhury and A. Mazumdar, "An accurate bound on tensor-to-scalar ratio and the scale of inflation," Nucl. Phys. B 882 (2014) 386 [arXiv:1306.4496 [hep-ph]].

[50] S. Choudhury and A. Mazumdar, "Primordial blackholes and gravitational waves for an inflection-point model of inflation," Phys. Lett. B 733 (2014) 270 [arXiv:1307.5119 [astro-ph.CO]].

[51] S. Choudhury, A. Mazumdar and S. Pal, "Low \& High scale MSSM inflation, gravitational waves and constraints from Planck," JCAP 1307 (2013) 041 [arXiv:1305.6398 [hep-ph]].

[52] S. Choudhury, A. Mazumdar and E. Pukartas, "Constraining $\mathcal{N}=1$ supergravity inflationary framework with non-minimal Kähler operators," JHEP 1404 (2014) 077 [arXiv:1402.1227 [hep-th]].

[53] S. Choudhury, "Constraining $\mathcal{N}=1$ supergravity inflation with non-minimal Kaehler operators using $\delta N$ formalism," JHEP 1404 (2014) 105 [arXiv:1402.1251 [hep-th]].

[54] P. Gondolo and G. Gelmini, "Cosmic abundances of stable particles: Improved analysis," Nucl. Phys. B 360, 145 (1991).

[55] A. Berlin, D. Hooper and S. D. McDermott, "Simplified Dark Matter Models for the Galactic Center Gamma-Ray Excess," Phys. Rev. D 89 (2014) 11, 115022 [arXiv:1404.0022 [hep-ph]].

[56] P. A. R. Ade et al. [Planck Collaboration], "Planck 2015 results. XIII. Cosmological parameters," arXiv:1502.01589 [astro-ph.CO].

[57] G. R. Dvali, G. Gabadadze and M. Porrati, "4-D gravity on a brane in 5-D Minkowski space," Phys. Lett. B 485 (2000) 208 [hep-th/0005016].

[58] S. Choudhury and S. Pal, "Primordial non-Gaussian features from DBI Galileon inflation," Eur. Phys. J. C 75 (2015) 6, 241 [arXiv:1210.4478 [hep-th]]. 
[59] S. Choudhury and S. Sengupta, "Features of warped geometry in presence of Gauss-Bonnet coupling,” JHEP 1302 (2013) 136 [arXiv:1301.0918 [hep-th]].

[60] S. Choudhury and S. SenGupta, "Thermodynamics of Charged Kalb Ramond AdS black hole in presence of Gauss-Bonnet coupling," arXiv:1306.0492 [hep-th].

[61] S. Choudhury, J. Mitra and S. SenGupta, "Modulus stabilization in higher curvature dilaton gravity," JHEP 1408 (2014) 004 [arXiv:1405.6826 [hep-th]].

[62] S. Choudhury and S. SenGupta, "A step toward exploring the features of Gravidilaton sector in RandallSundrum scenario via lightest KaluzaKlein graviton mass," Eur. Phys. J. C 74 (2014) 11, 3159 [arXiv:1311.0730 [hep-ph]].

[63] S. Choudhury, S. Sadhukhan and S. SenGupta, "Collider constraints on Gauss-Bonnet coupling in warped geometry model," arXiv:1308.1477 [hep-ph].

[64] S. Choudhury, J. Mitra and S. SenGupta, "Fermion localization and flavour hierarchy in higher curvature spacetime," arXiv:1503.07287 [hep-th].

[65] A. De Felice and S. Tsujikawa, " $f(R)$ theories," Living Rev. Rel. 13 (2010) 3 [arXiv:1002.4928 [gr-qc]].

[66] T. P. Sotiriou and V. Faraoni, " $f(R)$ Theories Of Gravity," Rev. Mod. Phys. 82 (2010) 451 [arXiv:0805.1726 [gr-qc]].

[67] N. Kanekar, V. Sahni and Y. Shtanov, "Recycling the universe using scalar fields," Phys. Rev. D 63 (2001) 083520 [astro-ph/0101448].

[68] V. Sahni and A. Toporensky, "Cosmological Hysteresis and the Cyclic Universe," Phys. Rev. D 85 (2012) 123542 [arXiv:1203.0395 [gr-qc]].

[69] V. Sahni, Y. Shtanov and A. Toporensky, "Arrow of time in dissipationless cosmology," Class. Quant. Grav. 32 (2015) 18, 182001 [arXiv:1506.01247 [gr-qc]].

[70] S. Choudhury and S. Banerjee, "Hysteresis in the Sky," Accepted in Astroparticle Physics, [arXiv:1506.02260 [hep-th]].

[71] S. Choudhury and S. Banerjee, "Cosmic Hysteresis," arXiv:1512.08360 [hep-th].

[72] S. Choudhury and S. Banerjee, "Cosmological hysteresis in cyclic universe from membrane paradigm," arXiv:1603.02805 [hep-th].

[73] S. Choudhury, M. Sen and S. Sadhukhan, "Can Dark Matter be an artifact of extended theories of gravity?," arXiv:1512.08176 [hep-ph].

[74] S. Choudhury and S. Panda, "COSMOS-e'-GTachyon from String Theory," arXiv:1511.05734 [hep-th].

[75] S. Choudhury, "Inflamagnetogenesis redux: Unzipping sub-Planckian inflation via various cosmoparticle probes," Phys. Lett. B 735 (2014) 138 [arXiv:1403.0676 [hep-th]].

[76] P. S. B. Dev, A. Mazumdar and S. Qutub, "Connection between dark matter abundance and primordial tensor perturbations," arXiv:1412.3041 [hep-ph]. 